\title{
Martial Arts and Well-Being
}

Martial Arts and Well-Being explores how martial arts as a source of learning can contribute in important ways to health and well-being, as well as provide other broader social benefits. Using psychological and sociological theory related to behaviour, ritual, perception and reality construction, the book seeks to illustrate, with empirical data, how individuals make sense of and perceive the value of martial arts in their lives.

This book draws on data from over 500 people, across all age ranges, and powerfully demonstrates that participating in martial arts can have a profound influence on the construction of behaviour patterns that are directly linked to lifestyle and health. Making individual connections regarding the benefits of practice, improvements to health and well-being - regardless of whether these improvements are 'true' in a medical sense - this book offers an important and original window into the importance of beliefs to health and well-being as well as the value of thinking about education as a process of life-long learning.

This book will be of great interest to a range of audiences, including researchers, academics and postgraduate students interested in sports and exercise psychology, martial art studies and health and well-being. It should also be of interest to sociologists, social workers and martial arts practitioners.

Carol Fuller is a professor of education and Senior Fellow of the Higher Education Academy at the Institute of Education, University of Reading, Reading, UK.

Viki Lloyd is the director of the Reading Acupuncture Clinic and the lead instructor of Sei Shin Kan School of Karate and Reading Chenjiagou Taijiquan GB, Reading, UK. 



\section{Martial Arts and Well-Being}

Connecting Communities and Promoting Health

Carol Fuller with Viki Lloyd 
First published 2020

by Routledge

2 Park Square, Milton Park, Abingdon, Oxon OXI4 4RN

and by Routledge

52 Vanderbilt Avenue, New York, NY 10017

Routledge is an imprint of the Taylor \& Francis Group, an informa business

(C) 2020 Carol Fuller and Viki Lloyd

The right of Carol Fuller and Viki Lloyd to be identified as authors of this work has been asserted by them in accordance with sections 77 and 78 of the Copyright, Designs and Patents Act 1988.

The Open Access version of this book, available at www. taylorfrancis.com, has been made available under a Creative Commons Attribution-Non Commercial-No Derivatives 4.0 license.

Trademark notice: Product or corporate names may be trademarks or registered trademarks, and are used only for identification and explanation without intent to infringe.

British Library Cataloguing-in-Publication Data A catalogue record for this book is available from the British Library

Library of Congress Cataloging-in-Publication Data A catalog record has been requested for this book

ISBN: 978-I-I38-2I355-5 (hbk)

ISBN: 978-I-3I5-44808-4 (ebk)

Typeset in Bembo by codeMantra 
This book is dedicated to the people in my life who support me unconditionally, encourage me when I need it and never fail to show interest in what I do - even when what I do is only ever likely to be of interest to me. The list of who these are is much too long to include here but special mention goes to my sister Joanne 'Little Button' Withey, Maria Danos and David Simmsy. Without their support, this book would never have been finished.

With love to you, Carol Fuller. 



\section{Contents}

List of figures and tables

1 Introduction

Part 11

Scope of the research and structure of the book 5

Part 26

Methods 6

Research design 6

Data collection 7

Sample 8

Survey 8

Interviews 9

Data analysis 10

Survey 10

Interviews 10

\section{Theories of learning behaviour and reality} construction - their value in understanding health and well-being

Introduction 12

Exploring individual attitudes and behaviour 13

Behaviourism 14

Social cognitive theory 15

Four main sources of self-efficacy 16

Self-efficacy and human health and well-being 17

Society and behaviour 18

Culture and group behaviour 20

Martial arts, health and well-being 21

Eastern philosophy and its relevance to martial arts 22

Chapter summary 26 
3 Teachers of martial arts

Introduction 27

Sample 27

Motivations for taking up a martial art 27

Physical 28

Health 28

Well-being 29

General 30

Health and health awareness 32

Well-being 34

Additional benefits 36

Culture 37

Community 37

Inclusion 39

Challenges to teaching martial arts 40

Value of teaching 41

Access to martial arts 43

Chapter summary 43

\section{Health and physical well-being and the teaching and}

\section{learning of martial arts}

Introduction 45

Sample 45

Motivations for taking up a martial art 46

Health 46

General physical well-being 48

Support for health: awareness and benefits 50

Health awareness 50

Health benefits 52

Teaching and learning 57

Learning and the martial arts teacher 59

Qualities in the teacher 60

Challenges to learning 61

Being a martial artist 62

Chapter summary 63

5 Well-being

Introduction 64

Sample 64

Martial arts and well-being 64

Well-being and confidence 66

Intellectual 69 
Learning 69

Culture and ethics 71

Social 72

Management of stress and the link to health and well-being 75

Chapter summary 82

6 Connecting communities and promoting health

Introduction 83

Motivations to learn 85

Perceived benefits of martial arts 88

Health benefits 88

Health awareness 89

Well-being 91

The social context of learning 94

The role of the teacher 95

Identity in martial arts 97

Connecting communities and promoting health 98

Chapter summary 100

7 Conclusion

Significance of the research 104

Recommendations for policy and practice 105

References

Index 



\section{Figures and Tables}

\section{Figures}

4.1 Graph showing attributes considered important in a martial arts teacher

4.2 Word cloud of key words used to describe a martial artist

\section{Tables}

1.1 Age range of survey sample

1.2 Geographical location of survey sample 9

1.3 Additional countries included in the survey 9

1.4 Age range of interview participants 10

3.1 Martial arts and levels of health awareness 32

3.2 Martial arts and health improvements 33

3.3 The impact on everyday life of participation in martial arts $\quad 35$

3.4 Additional benefits of martial arts practice 36

3.5 Attributes considered important in a martial arts teacher 42

4.1 Martial arts and levels of health awareness 51

4.2 Martial arts and perceptions of improvements in health 53

4.3 Attributes considered important in a martial arts teacher 59

5.1 Improvements to well-being since beginning martial arts 65

5.2 Other benefits to martial arts practice 66 



\section{Acknowledgements}

To the many martial artists, from beginners to experts, we thank you all for the time and insights you gave to this research. We are grateful to our friends, colleagues and fellow practitioners for their interest, insights, support and encouragement. In particular, we thank Professor Alan Floyd, Professor Suzanne Graham, John Gouk and Zhu Xi - for his inspirational writings. 



\section{Chapter I}

\section{Introduction}

\section{Part I}

It could be argued that the new millennium is one of global crises in terms of health and well-being. How and in what way can notions of lifelong learning, in this case that of martial arts, contribute to health and well-being and how do they impact, if at all, on everyday lifestyle and practice from the perspective of those who practice them? These are important questions, given the concerns of health and well-being dominating the 21st century. There is currently a growing global concern related to the impact of a range of health challenges and the demand on resources to tackle them. Could exercise, and martial arts specifically, offer a cost-effective source of support and help to these concerns? To illustrate, in 2015, global life expectancy had increased by 15 years from that of the 1960s (World Health Organisation, 2017); yet, at the same time, death rates from cardiovascular disease had increased by 41\% (Roth, Forouzanfar, Moran, Barber, Nguyen, Feigin, Naghavi, Mensah and Murray, 2015). To illustrate, one in four deaths in the United States(US), almost $38 \%$ of deaths of those under the age of 75 in the United Kingdom (UK) (Heart UK, accessed July 2017) and 31\% of deaths globally (WHO, 2017) were attributable to chronic heart disease or similar. Whilst deaths from heart disease have risen, it is perhaps unsurprising that most of these deaths occur within the top income countries (WHO, 2017). Clearly, lifestyle could be argued as having a bearing on this. Any number of searches will highlight the increasingly sedentary nature of these as well as the importance of diet, weight, smoking, alcohol consumption, blood pressure and diabetes, as well as physical activity in determining the risk of heart attack; indeed, the UK National Health Service (NHS, 2017) states that those who undertake no physical exercise at all are twice as likely to suffer a heart attack as those who do.

However, one of the biggest threats to health in the 21st century is diabetes, ${ }^{1}$ the disease the media often refer to as the 'silent killer.' While explanations for this rise are numerous, there is consensus as to the role that lifestyle plays. Globally, rates of those diagnosed with diabetes have doubled 
from 1980, with estimates of 422 million people living with diabetes in 2015 (WHO, 2016). The International Diabetes Federation (IDF) calculates that this figure will rise to 642 million by 2040 . With these sorts of numbers, it is clear to see why diabetes is now one of the four 'non-communicable disease' priority health areas for countries around the world (WHO, 2016). If left untreated, potential complications from diabetes are heart attack, kidney failure, blindness, nerve damage and amputation, as well as death. Alongside a serious impact on health and well-being, diabetes has a profound economic cost to national health services. For example, estimates suggest that costs to the British NHS for treating diabetes will reach $£ 16.9$ billion by 2035 (Hex, Bartlett, Wright, Taylor and Varley, 2012), most of which will be spent on preventable complications.

The cost implications of diabetes are important to consider because it is no longer the disease of those in the West. Indeed, China now tops the international rankings for the number of adults living with diabetes - 109.6 million in 2015 according to the IDF (2015). China has experienced a very clear and rapid increase, for example, from $1 \%$ of Chinese adults with diabetes in 1980 to $10 \%$ by 2008 (Hu, 2011) - a spike in rates that could be argued as mirroring its economic development. Hu (2011) notes that Asian countries now account for $60 \%$ of the global diabetes rates; yet, the escalating costs to support the health demands of the disease may mean that much of the economic gains currently being enjoyed via rapid urbanisation and globalisation could be lost to combating escalating health issues.

Whilst there are a multitude of variables and influences that are linked to causing diabetes, which are beyond the scope of this book to explore, lifestyle is a key important contributory factor for type 2 diabetes, particularly when linked to obesity and levels of physical activity (National Institute of Diabetes and Digestive and Kidney Diseases, 2016). Rates of obesity are said to have doubled in over 70 countries since 1980 (The GBD 2015 Obesity Collaborators, 2017), with some 603.7 million adults and 107.7 million children classed as obese in 2015. A person is considered obese if his or her body mass index exceeds 30 (with 18.5-24.9 being considered a healthy range) (WHO, 2006) and results from consuming more calories than are utilised via physical activities. Surplus calories are then stored as fat, so diets high in fat and sugar content are particularly problematic for this reason. Whilst obesity has no direct health consequence, it is a contributory factor in an extensive range of health conditions, including those mentioned earlier. In addition, issues of depression, low confidence and low self-esteem can often accompany obesity that adds further complexity to the challenges now faced. In a meta-analysis of 15 longitudinal studies with a total sample size of 58,745, a clear link between obesity and depression in adults was found and established as statistically significant (Luppino, de Wit LM, Bouvy, Stijnen, Cuijpers, Penninx and Zitman, 2010). Whilst the authors of this research note that it is not clear in which direction the causal relation works, that is, whether obesity 
leads to depression or vice versa, what is important is the connection between physical and mental health.

The importance of mental health is only now really entering the public psyche, in terms of its relevance to physical health. In 2012, for example, the British Government announced, 'No Health without Mental Health,' an intervention that set out to establish mental health as a priority area and acknowledging the need for parity in terms of the funding and resources required for both mental and physical well-being. In doing so, it highlighted the debilitating consequence of mental ill health in areas such as relationships, education and work (Department for Health, 2012) and, by implication, the consequences for economic productivity and public expenditure. Indeed, in an independent review of the Mental Health Act for the British Government, the cost of poor mental health to the UK, based on 'providing benefits, falls in tax revenue and costs to the National Health Service' (Stevenson and Farmer, 2017: 5), is estimated to be between f24 billion and f27 billion.

The issue of mental ill health is widespread. In a systematic review of data and statistics from countries within the European Union (including Iceland, Norway and Switzerland), 27\% of the adult population were found to have had one form of mental disorder within the past year - some 83 million of those were under the age of 65. The National Institute for Mental Health (2015), an American Federal agency, estimated that approximately one in five (or 43 million) Americans had diagnosable mental ill health in 2015. Whilst there are myriad reasons for these high levels of mental ill health, the National Institute for Mental Health (2015) makes a most salient point, and the one in which we should 'not lose sight of mental health [as so much] more than an absence of mental illness.' This point illuminates and exemplifies an important concern - that mental 'well-being' is very much connected to mental health and so is arguably a significant focus for government, business and individuals.

Mental well-being relates to an ability to manage the stresses of everyday life so that one can live a meaningful and productive life (as opposed to mental ill health, which can lead to serious disruption in a person's thinking and consequently ability to do these things). Chandola (2010), drawing on multiple measures, highlights how stress in the British workplace tripled to $25 \%$ from 1992 to 2006. Yet, mental well-being is not just the domain of those juggling demanding working lives. Increasingly, stress is an issue for children and young people too. In an article published in May 2017, The Guardian (a British newspaper) proclaimed 'a crisis in children's mental health' and the $\mathrm{UK}$ is not alone with this phenomenon; stress is an issue for students around the world (Houri, Nam, Choe, Min and Matsumoto, 2012).

Alongside a plethora of specific health challenges and concerns, it is estimated by the US government that by 2030, 1 billion people worldwide will be over the age of 65, ' 1 in every 8 humans on earth' (National Institute of Aging and National Institutes of Health, 2007: 4). A rapidly ageing 
population brings a whole range of additional challenges related to their care. Alongside this, changing demographics means that loneliness is an increasingly pertinent issue. In a review of the available research evidence looking at loneliness in the elderly, Age UK (2014) found that over 1 million people over the age of 65 said they were 'always' or 'often lonely,' with nearly half having only their pets or television for company. The social impact of this on well-being, the economic burden, in terms of care and a looming crisis in antibacterial medicine, highlights the importance of trying to find new and effective ways to tackle some of these issues.

Whilst the 21st century is facing challenges in terms of health and wellbeing, and on a global scale, alternative ways to address some of these issues are also a feature of this era. A quick browse of the internet, newspaper or magazine will inevitably include an article or advert for exercise, whether focused on its benefits, specific classes or an advert for sportswear. A report for the leisure industry suggested that the leisure industry was worth $\mathcal{E}^{4.9}$ billion in 2018 in the UK alone. This suggests that there is interest in health and well-being. Indeed, the Telegraph Newspaper (2017), quoting an Ipsos MORI poll of over 10,000 people, states that the number of vegans (those that only eat a plant-based diet) has risen in the UK by $360 \%$ since 2006. This growth is largely explained by a perception that a vegan diet is healthier. In their report of a study of the data collected from 89,000 Americans via the Health Interview Survey, the National Institute for Health (2015) noted that a third of those included in the survey sought alternative treatments to support their health care, with fish oil, meditation, yoga and chiropractitioner treatments being examples of those most frequently listed as sources of support for chronic pain, general health and stress. Indeed, alternative treatments can be extremely powerful in support of health and well-being. In a review of the benefits of activities like arts and dance, the British Arts Council (2014) highlighted their importance in tackling loneliness, anxiety and depression, whilst treatments such as acupuncture - an ancient art based on traditional Chinese medicine - have grown rapidly in popularity. Acupuncture is used to treat a whole range of conditions, including pain management, women's health and some psychiatric conditions, and has been highlighted as beneficial in numerous research studies (Park, Linde, Manheimer, Albrecht, Sherman, Smith, Sung, Vickers and Schnyer, 2008). In addition, in acknowledging its contribution to modern medicine, acupuncture is available on the NHS in the UK and is often covered under private health care insurance. Research with 330 acupuncture practitioners by Hopton, Curnoe, Kanaan and MacPherson (2012) for the British Medical Journal estimated that some 4 million acupuncture sessions were being carried out annually. They explain this as being partly the result of a growing evidence base highlighting its benefits in terms of outcomes.

Interest in ways to tackle a range of issues is growing; yet, what becomes apparent when searching for research on the health benefits of a broader range 
of alternative therapies and social activities is that the evidence is sparse, mixed and inconsistent in terms of the quality and rigour of the research reported. Much of the research that does exist focuses on the outcomes, in terms of value and improvement, but what is less clear is the question of how and why health and well-being are benefited in the ways recorded. In this book, we seek to address this gap by considering the health and wellbeing benefits from the perspective of those people engaged in some form of martial arts. Whilst it is difficult to pinpoint exact numbers and trends, there is consensus within academia, health and the media that interest and participation in martial arts have been growing in the West for some time.

Our rationale for considering martial arts as a focus for our research is, therefore, straightforward and based on four key areas. Firstly, martial arts are numerous and varied, and are accessible to all; they, therefore, have appeal to the very young, the very old and the very many in between. Secondly, the personal motivations and reasons to take up a martial art are also diverse, from wanting a hobby to support for health or to help with developing confidence, to name just a few. Thirdly, for those with an interest, martial arts have distinct pedagogic underpinnings which are strongly influenced and framed within the philosophies of the countries of origin. What all of this means is that martial arts have a range of dimensions and have the potential to offer a multitude of benefits to those who want them. This takes us to the fourth and final reason for the focus of our research, that of pragmatism. As a novice, beginner student of Taijiquan of 4 years and a martial arts teacher with over 30 years of experience, it made sense to focus on the things we had experience of but also, and more importantly, to explore an area of learning where access to participants for our research was possible.

\section{Scope of the research and structure of the book}

In the rest of this book, we explore the role of martial arts in promoting perceptions of health and well-being from the perspective of the 515 people that were included in our research. It is important to point out that our research is using martial arts as a rich and useful way of exploring perceptions of health and well-being and how these perceptions shape views of outcomes, from the standpoint of those who practice them. We are clear not to make any claims of causality in relation to health and well-being, nor do we attempt to understand how the various techniques adopted by different martial arts may contribute to perceived changes in health and well-being. Finally, it is important to point out that this book does not consider the development of martial arts through history, its growth in popularity as a phenomenon in its own right or the philosophical and cultural underpinnings of the different arts in any real depth. Each of these topics would be a study in its own right. What we do, though, is try and understand why people find undertaking martial arts valuable so that we can better understand the 
socio-psychological construction of health and well-being, as well as how and in what ways martial arts may be argued as contributing to those constructions. It is, therefore, hoped that this book will make a valuable contribution to discussion as to how individuals as well as society might consider managing, but also preventing, some of the prevalent health and well-being issues that are now challenging contemporary society, as well as the role of theory in explaining this.

The book is organised in the following way. This chapter has offered an introduction to the current social issues in which this book situates itself, namely, a growing global crisis in health and mental well-being. In Chapter 2 , we provide a theoretical framework for making sense of the data that are presented in subsequent chapters. Psychological theories of learning as well as more sociological theory are discussed very briefly. Martial arts are then considered in the light of these theories so as to make clear why research that focuses on this area has a broader applicability and can be particularly useful to explore, most notably, in relation to how and why the what people think matter. In Chapter 3, we present the data collected via survey and interview with the teachers of martial arts. Chapter 4 focuses on the data collected in relation to health as well as the experiences of teaching itself. In Chapter 5, the area of well-being is considered with a particular consideration on how and in what ways martial arts could be considered as supporting well-being. In particular, the ways that martial arts are considered as offering support to the intellectual, social and physical needs of some of the participants in this research are presented, along with the myriad ways these work to support mental well-being. In Chapter 6, the key ideas and themes presented throughout the empirical chapters are discussed in relation to the key theoretical ideas presented in Chapter 2 and in relation to the key questions this book set out to address. Finally, Chapter 7 summarises our key findings and the contributions to knowledge and then concludes by offering recommendations for future practice and research.

Information on how we carried out our research and who took part is highlighted in the following part of this chapter.

\section{Part 2}

\section{Methods}

\section{Research design}

The research reported here adopted a mixed methods design, drawing on data from a survey of 515 participants and interviews with a follow-up sub-sample of 40 of them. A mixed methods design was selected as the most appropriate approach to the areas we wanted to explore. This was also very much based on the philosophical and methodological frameworks of the researchers in 
that, whilst very much constructivist in our ontological assumptions, for this research, combining the use of both qualitative and quantitative data collection techniques offered a much greater scope for achieving a deep understanding of the issues we were interested in, providing a depth that would not be possible by using one approach alone. This epistemological stance can be considered as that which Cresswell and Piano-Clark (2007) note is one of pragmatism, whereby data are collected via 'what works best' to address the research questions we posed. For this book, the questions we pose are as follows:

How and in what way can martial arts be considered as contributing to health and well-being from the perspective of those that practice them?

Do martial arts impact on everyday lifestyle and practice, and if so, how, from the perspective of those that practice them?

\section{Data collection}

Data were collected via SurveyMonkey, an online survey tool, using both convenience and snowballing sampling techniques. These non-probability approaches to sampling are quite typical for research that targets a specific group of interest but where access to the required information that would allow directly targeting the research population is not possible (Babbie, 2002). Links to the survey were initially sent to martial arts clubs known to one of the researchers, and these were, in turn, asked to share them with their students and professional links. As a result, the survey was distributed via email and social media such as Facebook, as well as via paper copy for those without access to the internet. Data were collected over two months. These approaches enabled us to extend the geographical reach of the survey, as well as the numbers of those that took part.

The survey collected data that explored a range of areas connected to health and well-being as well as perceptions of how, and in what ways, martial arts did or did not contribute to these areas. Measures of well-being and anxiety were designed using those developed by the British Office for National Statistics. Questions also explored awareness of health and practice with respect to these. Primarily, the survey gathered data that considered perceptions, behaviour and practices. However, as a self-reported survey of participants with an expressed interest in the topic (evident via their participation), this survey is not intended to be considered as a representative study or one that is in any way generalisable.

From the survey, a sub-sample was selected to take part in interviews that followed up on a range of areas covered in the survey. Interviews were carried out over the phone, via Skype and FaceTime as well as via email for two participants who were deaf. These were recorded and later transcribed. 
Interviewing in this way enabled them to be carried out over a range of time zones and covered in greater depth number of areas that were included in the survey.

\section{Sample}

\section{SURVEY}

Five hundred and fifteen participants took part in the survey, and of these, $62 \%(n=313)$ were male and 38\% $(n=191)$ female. Eleven participants chose not to say. From Table 1.1, we can see that a range of ages took part in the study, with the greatest proportion being those between 36 and 55 years. Table 1.2 also shows the geographical spread, with the South East region being the most represented at $29 \%$ but where one-fifth of the sample were from outside the UK.

Of those who listed where they lived outside of the UK, 21 different countries are captured in the survey. The range and scope of these countries are included in Table 1.3.

Thirty-one per cent (160) of the sample are teachers of martial arts. This means that more than two-thirds of the sample did not teach martial arts $(68 \%, N=340)$. The range and type of martial arts practiced captured in the survey reflect the convenience and snowballing nature of the sampling approach. As a result, whilst 14 different martial arts are included in the survey, the most practiced by participants were Tai Chi $(51 \%, N=261)$, Karate $(43 \%$ $N=219)$, Qi Gong (21\%, $N=106)$, Kung Fu (6\%, $N=31)$, Aikido (4.5\%, $N=23)$ and Jujitsu $(3 \%, N=17)$. Other martial arts had nine or fewer respondents: Kendo, Taekwondo, Wing Chun, Ninjujisu, Capoeira, Escrima, Judo, Mixed Martial Arts, Muay Thai and Silat. Whilst the type of martial art is not a variable of interest to this research, it is worth noting the diversity of martial arts captured.

Table I.I Age range of survey sample

\begin{tabular}{lcc}
\hline Age & Frequency & Per cent \\
\hline 18 and under & 10 & 2 \\
$19-25$ & 26 & 5 \\
$26-35$ & 46 & 9 \\
$36-45$ & 114 & 22 \\
$46-55$ & 118 & 23 \\
$56-65$ & 87 & 17 \\
$65+$ & 92 & 18 \\
Prefer not to say & 1 & 0.2 \\
Total & 494 & 96.2 \\
Missing & 21 & 3.8 \\
Total & 515 & 100 \\
\hline
\end{tabular}


Table I.2 Geographical location of survey sample

\begin{tabular}{llcc}
\hline Location & & Frequency & Per cent \\
\hline Valid & Northern Ireland & 7 & 1 \\
& Scotland & 10 & 2 \\
& Wales & 7 & 1 \\
& East Midlands region & 32 & 6 \\
& London region & 23 & 5 \\
& Northern region & 8 & 2 \\
& North West region & 25 & 5 \\
& South East region & 152 & 29 \\
& South West region & 52 & 10 \\
& West Midlands region & 37 & 7 \\
& Yorkshire and the Humber & 8 & 2 \\
& Outside the UK & 96 & 19 \\
& Prefer not to say & 5 & 1 \\
& Total & 462 & 90 \\
Missing & & 53 & 10 \\
Total & & 515 & 100 \\
\hline
\end{tabular}

Table I.3 Additional countries included in the survey

\section{Europe}

France (2), Germany, Greece (2), Holland (4), Hungry, Ireland (4), Italy, Jersey, Norway (3), Poland, Portugal, Serbia, Slovenia, Switzerland

\section{North America}

The USA (I2), Canada (5)

\section{Rest of the World}

Algeria, Australia, Mexico, Taiwan

Note: Numerals enclosed in parentheses indicate the number of people.

In terms of experience, $61 \%$ of the sample had been practicing their martial arts for between 10 and $20+$ years: $15 \%$ for $10-15$ years $(N=77)$; $13 \%$ for $15-20$ years $(N=66)$ and $33 \%$ for $20+$ years $(N=167)$. Fourteen per cent had been training for less than ten years $(N=69)$ and $25 \%$ for less than 5 years $(N=124)$. The degree of training also varied from 'everyday' $(5 \%, N=22)$ to 'a few times a year' $(4 \%, N=21)$. Most of those included in the survey practiced between 'once' and 'twice' a week $(86 \%, N=402)$.

\section{INTERVIEWS}

A survey of a sub-sample of 40 were interviewed. These were selected from those who had indicated that they would be willing to be included in interviews. Participants were then organised into three groups based on: (1) being a martial arts teacher, (2) were 65+ and finally (3) based on their responses 
Table I.4 Age range of interview participants

\begin{tabular}{lc}
\hline Age range & Number of participants \\
\hline $26-35$ & 4 \\
$36-45$ & 3 \\
$46-55$ & 8 \\
$56-65$ & 3 \\
$65+$ & 15 \\
$75-80+2$ & 7 \\
\hline
\end{tabular}

to health and well-being measures within the survey. Of those interviewed, 25 were male and 15 female. The interview sample ranged in age, which is shown in Table 1.4.

In terms of their location, interviewees came from across the UK $(N=28)$ but also included those in the USA $(N=7)$, Norway (4), France (1) and Hungry (1).

\section{Data analysis}

\section{SURVEY}

Survey data were downloaded into the Statistical Package for the Social Sciences (SPSS) software. Data were analysed using SPSS with frequencies of responses and cross-tabulations used to explore the data. Frequencies showed us an overall picture of the data, whilst cross-tabulations enabled exploration of patterns and trends. Independent $t$-tests by gender and Analysis Of Variance (ANOVA) by other categories were also carried out on key variables of interest and within subsets of the data to explore relationships between responses and their strengths. Tests for internal consistency were carried out on some of the scaled measure data which related to well-being. A Cronbach's alpha test scored these as 0.9 on 12 items.

\section{INTERVIEWS}

Transcripts from interviews were analysed to identify key themes of interest, acquired through a process of iteration. Colour coding was used initially. This inductive and systematic approach allowed for an exploration of the data which permitted the themes that appeared relevant and significant to areas of health, well-being and more social aspects to begin to emerge and be categorised. A thematic analysis, in relation to the topics and themes that arose in the interviews, was carried out in relation to the discourse evident in relation to content and meaning and the specific areas of interest - identified in the literature as health and well-being. Categorising the data in this way 
allowed a long narrative to be condensed. Categories identified through this process were then created as codes in NVivo QSR 2010 (a software package that supports qualitative data analysis) for each of the three sets of interviews: 'martial arts teachers,' 'older people' and 'well-being.' It is worth noting, however, that the frequency of 'content' was not a specific aspect of this research. Instead, the analysis focused on the discourse and narrative so as to do what Kvale and Brinkmann (2009) suggest, namely, an exploration of the contradictory as well as an exploration of the similar. Therefore, analysis of frequently occurring themes was useful and provided some interesting starting points for the subsequent discussion in the following chapters.

To compare for similarity and difference within coding, transcripts were explored and compared by two coders. Although not framing this process as reliability testing in the strictest sense, by ensuring that the themes and categories within the coding have been developed 'with all conceivable precautions in place against...distortions and bias, intentional or accidental, and mean the same thing for everyone else who uses them' (Krippendorff, 2004: 211), the process we adopted did permit the establishment of credibility and confidence in the data analysis. Lincoln and Guba (1985) consider this process an important practice in establishing the trustworthiness of qualitative data analysis. Themes and sub-themes related to 'well-being,' 'health' and 'relationships' form the focus of this book.

All university ethical guidelines were complied with in carrying out this research. In particular, all those taking part gave informed consent and were assured of anonymity. The quotes presented in the findings chapters use pseudonyms and no identifying features are given.

\section{Notes}

1 Diabetes is a condition whereby the pancreas is unable to produce insulin, which is required to regulate sugar (glucose) levels in the blood.

2 Provided where specific age is given in the interview. 


\section{Theories of learning behaviour and reality construction - their value in understanding health and well-being}

\section{Introduction}

As established in Chapter 1, health and well-being is a pressing concern for the 21st century. In acknowledging this, a pertinent question as to whose responsibility is it to tackle this problem is the one that interests government, academics, the media and numerous health organisations. What is apparent is that the answer to this is not clear cut and debate is evident as to how much is society's responsibility and how much is that of the individual (Minkler, 1999). Certainly, the state has its part to play. This can be seen in its role in actively emphasising and teaching within schools on the importance of the health and well-being choices that people make, a significant feature of government health initiatives and school educational policies globally. Key to this international health and well-being challenge is providing access to information that promotes awareness and provides advice as well as offering links to practical support. Examples of the things now available include: information websites; television and media campaigns relating to specific issues such as strokes, smoking and obesity; free leaflets; drop-in clinics; mantras such as ' 5 fresh fruit and vegetables a day' as well as global health awareness days, for example, World Hepatitis B Day, World Antibiotic Awareness Week and World No Tobacco Day (WHO, 2017a). Although not without controversy in terms of the costs and choice of who to feature (Chapman and Rayner, 2012) increasingly, celebrities are also being used to support health campaigns and these have been found to be particularly effective in raising awareness (Kosenko, Binder and Hurley, 2016). With its National Health Service facing a funding crisis, in 2011, the British Government highlighted the need for a focus that was a more collective collaboration - deemed as essential - in supporting the broader challenge with its introduction of the 'Public Health Responsibility Deal.' This initiative encouraged businesses and organisations to pledge support for public health by offering much clearer and informative labelling on food and alcohol, as well as providing support for health and physical activities that promoted well-being within the workplace. As of 2017, 776 companies had signed up. 
Despite a range of ways that health and well-being is supported at the macro and meso levels, the engagement level of individuals varies. Before considering some of the ideas that might help with understanding engagement, as well as attitudes and behaviours that shape this engagement, it is first worth highlighting a key barrier to accessing the practice and activities that support these areas, regardless of motivations, that is poverty, a key barrier. Social structures inevitably create limitations for some, regardless of hopes and intentions. Poverty is obviously a fundamental factor but can only be touched on very briefly here. However, needless to say, finding resources to pay for healthy food and engaging in activities that encourage and promote health and well-being, as well as locating the support often needed for specific health care needs, are challenging for those with low incomes, 'even in countries that have universal healthcare' (Loignon, Hudon, Goulet, Boyer, De Laat, Fournier, Grabovschi and Bush, 2015: 1).

Yet, ideas about health and well-being are also cultural, where attitudes and perceptions can vary quite significantly within and across groups, even within the same societies (The Lancelet Commission, 2014). To illustrate, research with 95 women in rural states in the United States of America (USA) found that culture was important in explaining the attitudes towards healthy eating of those living in remote communities, where large, calorific meals were valued highly and healthy foods considered expensive (Seguin, Connor, Nelson, LaCroix and Eldridge, 2014). Within the young, time is perceived as the biggest barrier to supporting healthy eating and lifestyles. For example, an Eating and Activity Survey with 2,287 young people in Minnesota found that most of those surveyed believed that healthy eating took too much of their time (Escoto, Laska, Larson, Neumark-Sztainer and Hannan, 2012). Whilst time is not necessarily a concern of the young alone, the digital age is the touchstone of their generation and the one that they have embraced. Yet, the digital age is also, Levitin refers to, one of 'information overload' (2014). Within this context, time is needed for sifting and sorting an abundance of often competing sources and material. Clearly then, whilst structure matters when considering factors that impact on ability to engage fully with practice that supports health and well-being, perceptions and attitudes are also strongly framed within the sociocultural context. However, understanding the frameworks people draw on when making health and lifestyle decisions is not easy or clear cut. Multiple theories offer competing ideas as to what matters and why. In the following sections, we consider some of these briefly before reflecting on how and in what ways these are useful when researching the field of martial arts.

\section{Exploring individual attitudes and behaviour}

An attitude usually reflects a view, belief and/or emotion towards something and therefore often underpins the way we respond to people and situations. 
In trying to understand why people behave the way they do, it is important to consider how attitudes are shaped in the first place. This is a relevant consideration, not least because if lifestyle links to health and well-being why is it that for vast swathes of people, these are not conducive for healthy societies. Understanding how attitudes can be influenced and changed at the micro level becomes pertinent.

\section{Behaviourism}

For behaviourists such as Skinner, actions can best be explained as being the consequences of Thorndike's 'law of effect' (1953: 60), that is, the consequence of reward and punishment. For Skinner, behaviour is considered as learned; behaviour that is rewarded gets reinforced and strengthened and behaviour that is punished often dies out. Behaviour learned in this way is what Skinner referred to as conditioned behaviour or Operant Conditioning as it is generally known.

Operant conditioning is a theoretical idea developed to explain actions and is based on research studies with rats. The rats in Skinner's research received unpleasant electric shocks as they moved through a box. However, the rats quickly learned that knocking a switch turned off the electrical supply and as a result, they were able to avoid the discomfort. They, then, began to adapt their behaviour and to head straight for the lever when placed back in the box. Clearly, by modifying their behaviour, they were able to avoid an unpleasant experience (McLeod, 2007). This breakthrough was an important development in understanding human behaviour and indeed, the value of operant conditioning theory is intuitively obvious. For example, if we put our hand on a hot kettle, it will burn and so we will avoid doing this again. Equally, a child that is punished for not completing their homework is more likely to complete it next time. Behaviour and actions that produce a direct or indirect effect that makes us feel good, for example, praise when we do something well, or feeling warm and sated after a delicious meal, mean that the behaviour is much more likely to be repeated.

The influence and application of operant conditioning are evident through a plethora of behaviour modification programmes, programmes designed to change negative or destructive thought patterns and behaviour by 're-programming', that is, re-conditioning the mind to think and act in another way. Examples of the conditions and behaviours these are used with include addictions such as alcoholism and smoking, anxiety and phobias as well as obsessive-compulsive disorder (OCD) and attention-deficit hyperactivity disorder (ADHD). Whilst Skinner's theory is primarily critiqued for the inadequacy of a framework based on animal behaviour being applied to humans (Viruás-Oretega, 2006), it is considered as successful in treating phobias and neurosis (Naik, 1998). Research shows that it achieves very good results for conditions such as personality disorder (Matusiewicz, Hopwood, 
Banducci and Lejuez, 2011) and drug and substance abuse (McHugh, Hearon and Otto, 2010) as examples.

It is suggested that partly the success of behaviour modification programmes in changing behaviours in specific areas results from the fact that it focuses on behaviour where there are direct and observable external outcomes. It is less successful when behaviour is the result of more internal responses such as hunger (Viruás-Oretega, 2006) or emotions, such as anger. Internal stimuli can be very powerful and lead to unpredictable behaviour. Skinner's theory is also critiqued for its lack of consideration on the role of the environment on behavioural outcomes, for example, when hungry and poor. Behaviour, therefore, needs to be considered within its context as well as the underpinning attitudes and belief that are shaped by the environment in which the behaviour occurs.

\section{Social cognitive theory}

Social cognitive theory (previously referred to as social learning theory) is a theory of behaviour developed by Bandura in 1977 (Mcleod, 2016). According to McLeod (2016), Bandura develops and enhances the behavioural theories based on conditioning by introducing a focus on the processes that mediate stimuli and their responses, as well as including a consideration of the importance of the environment as a means of observational learning. The environment is key to Bandura's ideas about how children learn to behave because the environment is innately social and, therefore, provides an abundance of people to observe and learn from. This 'modelling' (or role modelling) is an important aspect of his work.

For Bandura, children learn from observing and then imitating the people around them. The models they chose to imitate will often reflect similarities they perceive to themselves (as opposed to someone they would like to be like) and can be family, friends, those in the media or people they interact with in their everyday lives. Watching how these models act and the consequences of the behaviour they observe is important in determining which behaviour is adopted and which is not. Similar to Skinner's ideas, behaviour that is adopted is that which is reinforced by reward and/or punishment, reward and punishment that can be internal or external, positive or negative.

Bandura, Ross and Ross's (1961) classic 'Bobo doll' experiment illustrates the idea of learned behaviour very clearly. In this experiment, 72 children aged between three and five years were exposed to either aggressive or subdued behaviour (in relation to play) from an adult. The research found that the behaviour of those who were exposed to the aggressive behaviour was markedly similar to what they were exposed to, whilst those who experience the subdued behaviour or, who were in the control group, did not display aggressive behaviour at all. Even more interestingly, the children placed in the subdued adult group displayed much calmer behaviour than those who 
were in the control group. Whilst it could be argued that an experiment such as this cannot help explain all the nuances of human behaviour or behaviour that is not the result of observing behaviours in others, it does offer powerful insight and a tantalising window into understanding how attitudes and beliefs are developed, which, in turn, is useful for thinking about those connected to health.

Indeed, Bandura has written extensively on the ways that social cognition theory helps explain health and the practices connected to it. He focuses on what is referred to as the 'demand side' of a health model, that is, 'helping people stay healthy through good self-management of their health habits' (Bandura, 1998: 624). A key concept underpinning Bandura's ideas is that of self-efficacy. Self-efficacy refers to an individual's beliefs about their abilities to exert influence and control over the things that affect and shape their lives. Bandura notes that those with high levels of self-efficacy will see difficult tasks and events as challenges to be mastered, whilst those with low levels will avoid challenging situations and obstacles, believing that they have little chance of success to overcome them (Bandura, 1994). Self-efficacy does not operate in isolation but is strongly mediated by environmental factors. How well an individual can manage these demands can have a significant impact:

the demands of everyday life...[as well as regulat[ing] motivation by determining the goals people set for themselves, the strength of commitment to them and the outcomes they expect for their efforts. Belief in the power to produce effects determines how long people will persevere in the face of obstacles... and how much stress and depression they experience in coping with taxing environmental demands.

(Bandura, 1998: 625)

Self-efficacy is the major determinant of action for Bandura and is also the primary mechanism that regulates motivation. An understanding of the factors that impact on levels of self-efficacy is important to consider.

\section{Four main sources of self-efficacy}

Bandura (1998) suggests that there are four main sources of influence on levels of self-efficacy, and these are summarised very briefly below:

1 Mastery experiences: where self-efficacy and resilience are developed through perseverance. Success that comes too easily means that when failures occur, discouragement is more likely. Too many failures before any sense of achievement is established can also have negative consequences.

2 Social models: seeing people similar to oneself achieve success creates a sense that such success is possible for oneself too. Through the way they behave and what they say, social models teach their observers skills and ways to deal with the demands of their environment. 
3 Social persuasion: persuading people that they have the skills and capabilities to succeed means that they are much more likely to keep trying, even when experiencing the challenge of a setback. Social persuasion is also about ensuring that individuals are given opportunities to develop at the right pace and not placed in excessively challenging situations too soon, which can increase the likelihood of failure.

4 Stress reactors: people assess their physical and emotional states and make judgements based on these. They perceive aches, pains and fatigue as indicators that they do not have the strength and stamina to succeed. Reducing people's reactions to these stress indicators means that you can alter how they assess their physical state.

For Bandura (1998), models of health behaviour (and by association wellbeing) that do not include a model of efficacy have no explanatory and predictive power. Ideas of efficacy can be directly attributable to the behaviours and intentions linked to health and this is discussed briefly in the next section.

\section{Self-efficacy and human health and well-being}

As noted earlier and in Chapter 1, much (but not all) of physical health is the result of lifestyles and habits. For Bandura (1997), the environment is an important aspect of these lifestyles. He argues that people are suffering and dying from preventable physical and health-impairing conditions. In his book Self-Efficacy in Changing Societies, Bandura presents a whole list of the most obvious of these health-impairing conditions:

- Nutritional habits - cardiovascular disease

- Sedentariness - weakens cardiovascular capabilities and vitality

- Cigarette smoking - major health hazard for cancer, respiratory disorders and heart disease

- Alcohol and drug abuse - disabilities and loss of life

- Sexually transmitted disease - serious health consequences

- Physical violence - maimed or life cut short

- Other activities that are fraught with physical risks as well as dysfunctional ways of dealing with stressors that produces wear and tear on the body

(Bandura, 1997: 25)

With respect to the environment, he notes that industrial and agricultural practices are injecting carcinogens and harmful pollutants into the air we breathe, the food we eat and the water we drink, all of which takes a heavy toll on the body' (1997: 25). Bandura argues that changing habits is key to promoting healthier lifestyles and it is self-efficacy that determines health-promoting behaviour. Efficacy in this domain is determined by an individual's assessment of their ability to deal with stressors - which directly impact on their health - as well as their sense of how much control they have 
over the behaviours that impacts their health. As Schwarzer and Fuchs (1997) note, many people try to cope with stress by regulating their emotions via their engagement in activities that damage health, for example, smoking or taking drugs to calm down when upset. Therefore, in Bandura's terms, when thinking about efficacy with respect to health, an individual with low levels of efficacy will see little point in changing their lifestyle if they believe that it will make little difference or they will fail. It is crucial, then, to find mastery over stressors and their associated behavioural responses.

In considering the role of efficacy, how and what promotes efficacy in some instances and not in others, as well as what supports the development of resilience to deal with obstacles/failures in one person but not others, even those within the same family, is not clear. Not everyone would agree that levels of efficacy are important in determining behaviour. Indeed, Dweck (2000) argues the opposite using education as an example. Dweck disputes the view that confidence is the panacea to success in behaviour, finding that the brightest, most successful girls educationally, were also the most vulnerable. In her research, high achieving girls very much perceived their failures as the result of their own flaws, which impacted significantly on their self-confidence. This suggests that efficacy alone is not enough to explain outcomes.

However, academic would most likely agree with Bandura (1997) that health habits and lifestyle choices are primarily formed during childhood and adolescence, with attitudes to eating and nutrition, physical exercise, fitness and leisure practices, as well as self-management around the avoidance of risky behaviour (Bandura, 1997), and are strongly framed by the messages and practices that are experienced and observed. In this sense, environment clearly does matter. Yes, whilst Bandura's theory considers efficacy at the level of the individual, it appears less useful when considered in connection to the role of collective beliefs and behaviours, in terms of cultural norms and values.

\section{Society and behaviour}

Human beings are intrinsically social and historically came together in groups for a range of reasons, including those that are completely functional, for example, to distribute the work needed to survive (such as food production, defence/protection, house building and then later, as societies developed, more specialised skills) as well as creating a sense of belonging (Durkheim, 1997). Creating a sense of solidarity is important to healthy societies. Through collective consensus, norms, values and expectations are established which regulate behaviour and allow cohesive societies develop. Without social cohesion, order breaks down and anarchy and anomie results (Durkheim, 1997). The importance of social cohesion has received increased attention in the 21st century and well-being appears as important within this social construct, alongside human rights and tolerance. For example, for the 
Council of Europe (2004), social cohesion is that which promotes the welfare of its members, whilst for the Organisation for Economic Co-operation and Development (OECD) the 'well-being of all its members' is included in its definition.

Social cohesion emerges in a number of ways but primarily, it is through processes of socialisation that the socially agreed values, expectations and practices become normalised and accepted or, at the very least, complied with. However, values and norms are not universal across social groups nor even across whole societies. For example, belching after a meal in one context would be considered the sign of an enjoyable and satisfying meal, whilst in another the height of bad manners. In addition, expectations regarding dress, language and behaviour vary considerably depending on age, gender, religion and profession. Underpinning all of these differences is the notion of culture. Culture refers to 'the ideas, customs and social behaviour of a particular people or society' (English Oxford Dictionary, 2017) and is very much a social construction; that is, culture is created and maintained by society and does not exist independently of society.

For social constructionists such as Berger and Luckmann (1966), human beings do not have the same biological drives as other species to determine how to interact with their environment. Social order is, therefore, necessary to ensure that their interactions are successful and sustainable. The social order that necessitates the successful co-existence of individuals and groups is created through human activities - activities that become reinforced over time, through habitualisation, and then institutionalised (Berger and Luckmann, 1966). Habitualisation is an important focus for post-modern sociologists, who see much of our behaviour, beliefs and values as embedded in habit and repetition. For example, whilst we learn to drive a car, clean our teeth and use implements to eat, soon after doing so the practice becomes automatic, tacit and we do not need to talk ourselves through the steps of each. Using these ideas, health, well-being and the practices that support them could be understood as resulting from the ways that they are constructed by different social groups, along with the ways that the habits and practices associated with them are reinforced. Beauty is a good example of what is considered beautiful in one context may not be so in another. Strongly contrasting views in relation to weight illustrate this well also.

However, for Bourdieu and other neo-Marxists, culture should also be considered as something that is highly structured and intrinsically bound up in the interests and values of differing social groups, as well as the broader ruling elites. In particular for Bourdieu (1997), habits and tastes are very much the product of the economic resources available to a family as well as the environment in which one is socialised. Under this premise, 'tastes' for food and eating habits are shaped by what money is available, in terms of the types of foods one can afford to buy and thus eat. The same is true for lifestyle practices that support health and well-being; 'taste' for swimming, for 
example, will result from having experienced swimming, which is, in turn, only possible if there are resources to pay to learn how to swim, to pay for transport to get to a pool for a swim as well as buy the appropriate clothing that makes swimming possible.

Applying Bourdieu's ideas to health and well-being, access to the practices and resources that support the promotion of health and well-being are unequal, suggesting that those with the least economic resources will also experience the poorest levels of health and well-being. To illustrate the validity of this point, death rates from all major causes for men aged between 24 and 64 years in England and Wales are much higher for those from manual backgrounds than those from non-manual backgrounds (The Office for National Statistics, 2009). Whilst not perfectly correlated, type of job links to levels of income. The rates of incidence and death for diseases such as cervical cancer are highest in countries which are the poorest, with eastern and western Africa having the highest rates, compared to northern American and western Asia, which have the lowest (Dutta and Marquez, 2012). In the USA, the number of people suffering with depression is significantly higher amongst those living in poverty (National Centre for Health Statistics, 2014) as opposed to those that do not. Poverty also exacerbates poor health and well-being because of unequal access to the resources that supports care and treatment, such as private and complementary treatments as well as medications. In the USA, for example, $10.7 \%$ of the total population of those under the age of 65 years did not have medical insurance (National Centre for Health Statistics, 2017), whilst more than two billion people in low- and middle-income countries lack access to essential medications (Leisinger, Gorabedian and Wagner, 2012). Structure is, therefore, important, as is the habitualisation of traditions and practices that are embedded in these social structures.

\section{Culture and group behaviour}

Whilst sociologists such as Beck (1992) would argue against society being structured, if we accept that society is, then culture is important. At its most basic, culture is a reflection of the reification of ideas that connect to, and underpin, the practices that create social order amongst groups. If we accept this definition, then considering group behaviour is useful in trying to understand what a society values. Sociologists consider the myriad social goods that are the result of collective activities. To illustrate, Durkheim (1995) saw religion as crucial to cohesive societies. This was because the act of regular worship brought people together, reinforcing bonds, preparing people for social life via the discipline imposed for particular rituals, providing moral codes to inform behaviour, offering a source of support and comfort at important points and, at a more existential level, giving meaning to life. Other group activities can also offer similar benefits, such as attending football and other sporting occasions and music concerts. These social practices also involve a collective gathering, singing and, for the most devoted, a form of fervent worship. 
Collective behaviour has been highly important to societies across the millennia, with ritualistic dancing considered as having an evolutionary function that enabled larger groups of people, that is, those that were not kin - to live together (Ehrenreich, 2006). Indeed, Ehrenreich suggests that dance was far more effective than language in binding groups together, is rhythmically pleasurable and can be enjoyed as either participant or observer. As McNeill (1995) notes, the rhythmic patterning of ritualistic movement, such as army drill and dance, has a direct influence on both physical and psychological levels. Whilst research has not been able to look at the impact of these types of activities on the neurological system, he speculates that 'the rhythmic input through muscles and voice...may provoke echoes of the foetal condition when a major, perhaps principal external stimulus to the developing brain was the mother's heartbeat' (1995: 7). Goodger (1986) agrees that the intense, shared nature of sports participation generates a sense of a shared identity, partly as a result of the common rules and values associated with particular sporting groups. For Goodger (1986), the ritualistic behaviour related to sports is most often the medium through which this identity and sense of commonality is expressed. Thus, rhythmic and collective group behaviour promotes feelings of well-being as well as a sense of belonging that contributes in important ways to health.

\section{Martial arts, health and well-being}

According to the Cambridge on-line dictionary, a martial art can be considered as 'a sport that is a traditional Japanese or Chinese form of fighting or defending yourself.' However, such a definition fails to do justice to the depth and breadth of the multiplicity of arts that the term 'martial' could be applied to. Martial arts typically have their own history, culture and etiquettes with practices that vary in form and technique. As noted in Chapter 1 though, these aspects are not part of the focus of this book. However, if martial arts could be considered as encompassing ritual and if, as Collins notes, 'interactive ritual is the key to micro-sociology, and micro-sociology is the key to that which is much larger' (2004: 3), then research that focuses on the motivations for taking up a martial art, as well as the benefits that such an activity offers, is both valuable and insightful. In terms of the health and well-being, there is a plethora of research that looks at the benefits of different types of martial arts in areas such as confidence, posture, flexibility and stress, to name just a few. Martial arts and health will be covered in much more depth in Chapter 4. However, it is worth noting that martial arts are practised by both the very young and the very old as well as everyone else in-between; hence, their diversity and inclusivity make them an interesting focus for research. Yet, to the best of our knowledge, research on the health and social benefits of martial arts tends to be small scale and specific, and, most often, is framed within particular academic and disciplinary discourse, such as health. Our research addresses this gap by adopting a multi-disciplinary approach to our 
research. By exploring the motivations, benefits and perceived outcomes of participation in martial arts, from the perspectives of those that take part, this mixed methods large-scale study also adds significantly to research in the area of health and well-being, and in an innovative and fascinating way.

\section{Eastern philosophy and its relevance to martial arts}

Whilst martial arts have been recorded from across the millennia and from places such as ancient Egypt and Greece, there is some consensus that the more enduring martial arts are primarily situated within early Chinese history. Whilst we have noted that this history is not part of this book, a brief introduction to some of the key philosophical ideas that have permeated Chinese society, and thus martial arts and their practice, is relevant.

Setton (2008) suggests that martial arts have their history in ancient Chinese military training but can be observed as distinctly merging with key philosophical tenants from Daosim and Ch'an (Zen) Buddhism to 'transform from mere techniques of self-defence to a wide variety of practices aimed at self-cultivation and personal well-being' (56: 2008). He notes that one of the earliest recorded references to the idea that martial arts was more than just a defence system is in the record of a conversation between the King of Yueh and a female warrior, where a clear connection can be observed with the Daoist Book of Changes and the key principals of martial training. He adds that this is even all the more striking because it is a female warrior that (a) has a rarefied audience with a king and (b) is lecturing him!

It is worth noting that despite the historical links between martial arts and Daoist and Buddhist philosophies, there is contention as to whether these ideas are relevant to the key martial arts. Cynarski (2017), for example, finds no overall consensus as to the philosophy of martial arts, highlighting the challenge of unpicking the distinctions between philosophy, religion and ethics. This mirrors well the broader, and more general, domain of academia where, arguably, absolute consensus is almost unheard of. That said, Wang and Stringer (2000) suggest that whether philosophical, religious or ethical, key ideas can very much be observed as underpinning lifestyles and cultures in the East. They add that the new 'millennium is marked by an increasingly interconnected world...creating opportunities for shared meaning and realisation of similarities among people, communities and cultures, previously separated by distance and ideology' (Wang and Stringer, 2000: 33). What this means is that an increasingly connected world also offers opportunities to exchange and share ideas and practice, including those connected to leisure and lifestyle. For these academics, Taoism is of particular influence on the realm of leisure in China but proposes caution in assuming that the leisure practices that transfer out of China are universal. They suggest that it is important to consider both the similarities and the differences when comparing similar leisure practices in China and North America, for example. Arguably, 
this is because different philosophies and beliefs will impact, not just on the practice itself, but also on the perception of how and why a practice is the way it is and what the benefits are. Yu and Berryman (1996) add further that this is partly because 'leisure' itself could be considered as conceptualised differently, depending on the cultural and geographic contexts. For Liu, Yeh, Chick and Zinn (2008) however, an understanding of leisure practice should be approached as that without cultural boundaries, that ideas are alike or different dependent on the individual perspective taken.

The martial arts most popular in Europe and North America vary between both hard and soft styles and are those that are most heavily influenced by Taosim, Confucianism and Buddhism (Burke, Al-Adwal, Lee and Audette, 2007). Wang and Stringer (2000) suggest that one aspect of leisure that could be considered as transcending boundaries of culture is that of 'wellness.' There is also a growing evidence base that martial art is a leisure activity that directly supports feelings of wellness (Burke et al., 2007). It is not the intention of this book to try and make links between philosophical ideas behind martial arts practice and health and well-being. That said, as many of the popular martial arts do have their inception in Asia, it is worth considering, at least in a very broad way, the philosophical beliefs connected specifically to wellness, underpinned in some of the ideas associated with martial arts.

Ideas associated with Zen and Taoism are those most readily connected to notions of wellness (Wang and Stringer, 2000). Japanese martial arts, such as Judo, Karate, Kendo and Aikido, focus on notions of stillness (Zen and Buddhism, accessed 2019). For practitioners of these arts, those that see them as more than just a sport, the deeper philosophical aspects are crucial to the learning of the associated arts. To illustrate, 'Bushido' teaches its students to understand the nature of your mind so as to find peace and self-mastery (Zen and Buddhism, accessed 2019). Bell (2008) found, in a review of literature, that the Zen techniques required from the hard-Asian martial arts were particularly important in building perceptions of resilience amongst the practitioners included in the research he looked at. This is perhaps unsurprising given that 'Zen' is, at its most basic and simple, a focus on mindfulness - to use popular, contemporary parlance. These practices, although not yet clearly understood or substantially evidenced in the health and science academic literature, do have an impact on specific health conditions. For example, Matchim and Armer (2007) published a review of literature that looked at the psychological benefits, alongside the impact on health, of patients with cancer. Their review found that, although clearly more studies were needed, mindfulness meditation appeared to have an important impact and therefore recommended as a potentially beneficial tool for nursing patients with cancer.

If learning control over the mind and the emotions is a fundamental principle in Bushido, then it makes intuitive sense to see how, and in what ways, such practices can be useful to everyday health and well-being. Indeed, as already noted earlier, there is a growing body of empirical evidence that 
highlights the physical health benefits of engaging in martial arts. A brief look at some of these shows a range of benefits. For example, in a randomised control trial (RCT) of 200 adults with a mean age of 70 , results showed that karate improved attention, resilience and motor reaction time for the participants in the research (Witte and Böckelmann, 2016). In another study, 456 participants over the age of 60 who had visited an emergency room in a hospital, as the result of a fall within a six-month period, took part in an RCT in Taiwan. The intervention study sought to explore the impact of Tai Chi on prevalence of further falling and found that there were statistically significant beneficial differences in the rate of falls for the Tai Chi experimental group (Hwang, Chen, Lee-Hsieh, Chien, Chen and Lin, 2016). Despite a growing body of evidence however, Pons van Dijk, Leffers and Lodder (2014) note that some of the underpinning methods of the published research are less than rigorous. On a more encouraging note though, they add that the suggestive themes emerging from their attempted systemic review of published research does point positively to benefits, and thus conclude by highlighting the important need of carrying out more research. That said, there is, however, a much larger evidence base for the soft martial arts. 'Soft' martial arts most often refer to - although not always - a style of martial art that is considered as more internal, as opposed to external.

Taoism/Daoism is most closely linked with Kung Fu and Taijiquan and is a philosophical approach that makes explicit connections between nature and the human body. Practices in these styles are designed to mirror the rhythm of nature and so the links to health and well-being are again intuitively clear. For example, at its most basic is the notion of Yin and Yang. Yin and Yang relate to ideas of complementary forces essential to, and for, life. Complementary, in this case, denotes oppositional. To illustrate, for something to be known, it must first be observed in reference to something else. Think of happiness, for example, can we know what happiness is without experiencing sadness? Would we be aware of darkness without the contrast with light? The fact that one cannot exist without the other is the fundamental principal of Taoism. Qi is the material/energy force that Yin and Yang manifest through. Taoist influence on these martial arts can be most readily seen in their practice, a practice that seeks to generate Qi using movements and form that replicates the principals of Yin and Yang. For Taoist such as Wong (2015), practice that draws on these principals will naturally support health and well-being because the focus is on increased physical and self-awareness. Research suggests that these types of practices have numerous benefits; for example, an RCT study on 120 healthy older people in China, who took part in a Tai Chi class three times a week for just under a year, showed increased brain volume linked to memory and learning and that the intellectual improvements they experienced were considered partly as a response to the social interactions that were part of the classes (Mortimer, Ding, Borenstein, DeCarli, Guo, Wu, Zhao and Chu, 2012). Other research highlights benefits 
to levels of anxiety and depression (Wang, Lee, Wu, Benson, Fricchione, Wang and Yeung, 2014; Sharma and Haider, 2015) as well as balance and the management of pain (University of Berkeley Wellness, accessed 2019). However, all of these research findings are very much focused on making causal connections between a practice and an outcome. What the individual benefits are, how they are perceived and how these influence practice are not considered in the way this book seeks to do, nor do they recognise the importance of the teacher to that learning. The 'supervision and guidance of a good teacher' (Siaw Voon Sim and Gaffney, 2018: 12) is considered as one of the three key features necessary to learn and develop as a martial artist, along with the self-discipline to train independently and an ability to comprehend and have insight into concepts (Siaw Voon Sim and Gaffney, 2018).

It is our contention that drawing on these broad Asian philosophical ideas is also useful for thinking about the nature of research or how we arrive at conclusion about the things we consider important. Zhu Xi, an ancient Chinese philosopher writing more than 900 years ago and most noted for his neo-Confucianism writing, suggests that all action must first be preceded by thought/intention (Li), that is, by the idea to do something (Jones and He, 2015). Using this principle at a more superficial level, as a key point of reference, it is worth exploring a little more how, and in what way, this very principle is helpful in providing an additional important framework, not just when seeking to understand what people do and why, but also in terms of providing guiding principles to the process of carrying out the very research that seeks to explore these areas.

Zhu Xi, whilst very much an advocate of Confucian ideals, was firmly in favour of the importance of perfecting the self. Thus, whilst he considered good governance as key to society as Confucius did, he did not believe that it should be at the cost of a lack of attention to personal development (Adler, 2014). For Zhu Xi, self-development, self-cultivation, inquiry and study were the clearest way to achieve 'authenticity' yet 'without teachers... it is impossible to seek out and obtain in one's body that which makes the hum an being the most rare' (Adler, 2014: 214). Thus, self-awareness, in both body and mind, was key to self-development, to 'completeness'; yet, such awareness was not possible without direction and guidance that was only possible through teaching.

From a research point of view, Zhu Xi was clear that it was only through dedicating oneself to the investigation of things, was it possible to extend our knowledge. Therefore, as intention (Li) is inexplicably bound up in every aspect of life from behaviours, 'relationships, cycles, processes, events, and things' (Stanford Encyclopaedia of Philosophy, 2015: 4), it was important to understand those intentions, the relationship between them and their consequences. He further suggested that to draw objective conclusions, one must first emerge through a process of self-learning. It is through this self-learning, and through the cycle of continuity, that this objective learning enhances and 
reinforces the self-learning. It is these ideals, then, that offer the deeper philosophical underpinnings to this research - that is, to draw broader conclusions about the nature of things, we much first reflect on what we have learned. Thus, to address the question that frames this book, the question of how and in what ways do martial arts impact on perceptions of health and well-being? we first ask the participants who took part in the research, to reflect on their experiences and to offer their perspective, through a range of questions, which allow them to explore and then share their own self-learning.

\section{Chapter summary}

In short, the ideas presented in this chapter provide the theoretical framework for the following empirical chapters. In this chapter, we have touched very briefly on some key ideas that are useful in considering how behaviour and lifestyle can impact on health and well-being. Specifically, this chapter explored theoretical ideas associated with the factors that shape actions to try and help us understand some of the psychological and sociological influences that are important to this area. Finally, why and in what way martial arts could be considered as useful in relation to health and well-being was introduced, first by considering the philosophical underpinnings to martial arts in a very broad and superficial way and second, by presenting some of the research evidence that indicates positive benefits for health and well-being connected to martial arts practice. Finally, we offer a clear positioning as to the relevance and rationale for exploring the focus of this book in the way that we have. In the subsequent chapters, the data collected through this research will be presented before being discussed in Chapter 6 in relation to the key ideas presented here. 


\section{Chapter 3}

\section{Teachers of martial arts}

\section{Introduction}

In this chapter, we present the views and opinions of the teachers of martial arts who were included in the research, considering their experiences of martial arts as well as their views and observations of the practices and motivations of those they teach. It takes a comprehensive overview of all of the key areas connected to this research that relate to health, well-being and 'being a teacher' from the perspective of those that teach.

\section{Sample}

Of the 515 respondents in the survey, martial arts teachers accounted for 160 of the sample. Of these, $77 \%(n=122)$ were male and $23 \%(n=37)$ female, predominately in the age range of 36-65 years. As reflective of the convenience sampling approach noted in Part 2 of Chapter 1 , Karate $(58 \%, N=92)$ and Tai Chi $(44 \%, N=71)$ were the martial arts that most of the teachers taught, although a broad range of martial arts were also practised. The majority of the teachers had been involved with one martial art form or another for more than 20 years $(84 \%, n=109)$. Geographically, $64 \%(n=112)$ of the teachers were based within the United Kingdom (UK) and 36\% $(n=47)$ outside of the UK.

In addition to the survey data, follow-up interviews were carried out with 14 teachers. The interview sample comprised four female and ten male teachers, ranging in age from 26 years to $65+$ years. Seven teachers were from England and seven were international, including three from the United States of America, two from Norway, one from Hungary and one from France. As the martial art world was frequently referred to as a small one, in terms of the connections between people and groups, no identifiers are included in this chapter with respect to gender, age or location so as to ensure the anonymity of those who took part.

\section{Motivations for taking up a martial art}

Martial arts teachers were asked to tell us why they had chosen to take up a martial art. This particular question within the survey was open-ended, 
allowing teachers to tell us in their own words. Their responses were then coded into the four dominant themes that emerged through a process of iteration: 'Physical,' 'Health,' 'Well-being' and 'General,' each of which is discussed next.

\section{Physical}

As might be expected, physical fitness was a main feature of the rationale for taking up a martial art for $17 \%(n=36)$ of the teachers, with references to support for/or improvement to flexibility, agility and mobility noted explicitly. This was also true for the motivations of some of the students that they taught. A general dissatisfaction with the gym was also an impetus for seeking something new, for those for whom physicality was important, as the following examples show:

I had poor posture and back pain

I needed to practice balance after an accident

I was bored with the gym. I wanted something with a bit more interest

\section{Health}

Health was also a motivator for some of the martial arts teachers. Comments coded as 'general health' and 'health support' accounted for $12 \%$ of the responses but also included things such as support for health in older age. The following examples illustrate concerns with health:

...you know, my health wasn't great, you know, I didn't feel that good and I knew that Tai Chi gave me that, that good feeling, um, and er I was like, I was gasping for it.

I have a family history of arthritis and wanted an exercise to keep my supple in old age.

I wanted to maintain my health into old age.

Support for chronic health conditions was also noted as important for both teachers and their students. For example, to help with on-going cancer treatment or in response to life-changing illnesses:

In 1990 when I was diagnosed with Hodgkin's Lymphoma.... I had to go through the radiotherapy followed by chemotherapy. During that time there was a gentleman from China who came ... so, he came over to visit his son and he started teaching Tai Chi, in the university.... I have learned that Tai Chi Chen can actually help me, kind of, work through the radiotherapy, the nausea 
I mean I've got people who've got Multiple Sclerosis and Parkinson's and...you know, I've got all kinds ... people that are in chemotherapy...

One respondent wrote that his working life has led to chronic back pain and that martial arts offered a comprehensive means to support his health needs. A similar example was given in one of the interviews:

I was working in a factory and moving a lot of heavy stuff around, transmission cases and doing that all day and I ended up, not, I didn't end up, my back started hurting quite a bit from doing that and I was looking for something comprehensive, for some comprehensive exercise that would help straighten that out. And that's when I decided to try the taekwondo.

\section{Well-being}

Stress was noted numerous times and emerged as a key theme, with martial arts posited as a potential channel to support and manage it. This theme was particularly evident in the students coming to their classes and is highlighted in the examples below:

I think that as, as time has gone on, now I think singularly people come, um, because they understand that stress is the biggest problem

...they're looking for a way of dealing with stress, I really think that that's it, so whether it's...you know they understand...you know, I think education you know, now people understand that stress is the biggest health problem

In addition, confidence was mentioned very frequently as a motivating rationale for taking up a martial art. Self-confidence and its link to stress and mental health were particularly interesting because of the myriad ways it appeared to impact. The following extract from an interview illustrates how confidence can impact on mental well-being very directly and how karate, in turn, provided a mechanism of support and transformation in dealing with it:

[I went along to my first martial arts class but] my teacher and my parents came to get me, they feared that it would make me more dangerous since I had these aggression issues. Because what happened was that I would really always answer in the only way that I knew how, which was violence....and er, one of my teachers, actually my maths and science teacher, he was er, he was a trainer in Karate and we were discussing natural reaction patterns and behaviours in the sciences and he started talking about Karate training and how that had changed a lot of his typical reactions patterns and er...so I went up to him after class and I spoke to him and he 
said actually we have a beginners class starting today so if you want to join me please do. So, I literally sloped off, without telling my mother, when I was thirteen and joined the beginners' class. And I'd been [when] I told my mum and she...she took it pretty well she said, yeah well you know, the bullying was years ago now, so this is probably fine, but, behave. And I've never looked back... absolutely, I mean I could have been stabbed, so Karate saved my life, in one way or another, because I was bullied to the extent of self-harm... and I attempted suicide...what happened with me was that I was pretty low on self-esteem when I started - Karate gave me at least the tools to cope for a while and the tools to focus, and er after a few years of training - six, seven, eight years - um I started gaining self-confidence when I realized from feedback on this and that I was actually quite skilled at what I did. But that took some time.

The association between well-being, confidence and physicality was very apparent in the data, particularly the connection between physical and mental well-being. This can be seen as illuminated well in the examples of two young men with physical disabilities below, from the perspective of one teacher:

[I teach a] blind lad for instance, he was getting bullied at school and he was lacking in confidence because these people were, well they were bullying him, so and he's so much more confident now. But for many... it gives them confidence. One of the other children that I work with, he's got dyspraxia and he's got this other condition where his muscles are overextended. His mother wanted him to come along, to help give him confidence in his body.

Martial arts as a means to manage specific mental health issues, specifically those including addictions to alcohol and drugs, were also referenced:

[I had a really hard time] a really bad time like drugs and alcohol and I got actually eating disorders and so I decided to go, and so for me it was a way of getting my life back on track because I knew that I was really very seriously going down the wrong path. So, that's actually what got me into it in the first place...

[a class which] I do, is more of a rehabilitation class for people who have had er, substance abuse problems or mental health problems...again to try and improve their confidence and again, to just try and get them physically fitter.

\section{General}

More general motivations included a desire for self-improvement through learning as well as wanting something that would offer layers to that learning, 
if desired, such as the theory, culture and philosophy associated with some of the arts.

...what I found, in particularly with Tai Chi, was that it's a combination of intellectual and physical involvement, so it's a total, sort of holistic, involvement so it's exercising the brain as much as the body...and that was the sort of thing I was looking for.

Interestingly, a sense of 'searching' for something was also noted. Several respondents mentioned how their much deeper commitment to their marital art came after a period of having given up for a while. Despite some very long absences in some cases, the teachers were drawn back into practicing it again.

Given that martial art is a club sport, not 'fitting in,' 'being good at' and 'not enjoying' team sports such as football were mentioned several times:

I wasn't particularly good at sports in school, I used to play...I used to play in goal...I didn't enjoy the running around in the freezing cold, I just didn't enjoy it, you know, and so they...they put me in goal, so I stood around in the freezing cold anyway, but I was the goal keeper and then come the cricket season I was the wicket keeper as well...I just didn't enjoy it really, you know, I wasn't particularly good at it.

I was never good at sports per se, you know football and track athletics or whatever, and so, yeah, when I started it was one of the few things where I felt...didn't feel weird...[it was] the thing I was comfortable with

Perhaps unsurprisingly, 'other people' featured significantly in how these teachers first encountered martial arts - either because a family member signed them up or took them along to a class. A desire to be 'just like' the artists from the movies clearly highlights the importance of role modelling. For example, Bruce Lee and David Carradine were frequently mentioned, as were fighting/Kung Fu movies more generally. Finally, self-defence was an important factor for $12.5 \%$ of the teachers and was also noted as a clear reason why some students - and in particular women - join their classes. The following offers one example:

...I'm quite a shy person, I used to be quite shy and I suppose walking past people, for instance, I would put my head down, now I don't. I feel confident that actually I'm OK, I'm, this is who I am and I have the confidence in the back of my head that if someone was to approach me, especially if it was in an aggressive manner, I would know that, how to actually get out of the situation really. So it's given me confidence within the street I suppose and a lot more confidence too because you're, when you're teaching you're having to approach people and you're having to work together to do things and yeah, it just, it has definitely given me confidence. 
It is clear that motivations for seeking and then starting a martial art are very varied but inherent in the multiplicity of motives is a desire and need of something - whether to be someone else, to be fitter and healthier or simply challenged and happier. However, what can also be inferred from the data presented here is that there is clearly a recognition within martial arts teachers that whilst for some people, their introduction to marital arts is almost serendipitous, most come for a reason and in response to an identified need - however basic this need might be considered to be - and because of a sense that martial arts can offer support for that need.

\section{Health and health awareness}

Participants of the survey were asked to indicate how more or less aware they were of various areas connected to health and what, if any, improvements they experienced in these areas, based on this awareness. The responses of those that completed these questions are depicted in Tables 3.1 and 3.2.

In Table 3.1, we can see that martial arts is considered to be contributing to much greater awareness of issues connected to general health, posture, wellbeing, flexibility and balance for almost three quarters of those that responded. In addition, greater awareness of rest and relaxation, diet and exercise, is also noted. Although only relevant to a much smaller proportion, more awareness of smoking, caffeine, sugar and alcohol consumption is also noted.

Table 3.2 highlights areas that are considered as having improved because of martial arts. From this, improvements are noted in all areas but particularly in the areas of balance, flexibility, levels of exercise, well-being, concentration

Table 3.I Martial arts and levels of health awareness (presented in the order items are listed in the survey)

\begin{tabular}{llll}
\hline & Less aware & $\begin{array}{l}\text { Neither more } \\
\text { nor less }\end{array}$ & More aware \\
\hline General health & 0 & $7.5 \%(12)$ & $74 \%(116)$ \\
Posture & 0 & $5 \%(8)$ & $77 \%(123)$ \\
General diet & $0.5 \%(1)$ & $33 \%(53)$ & $48 \%(76)$ \\
Sleep patterns & $0.5 \%(I)$ & $43 \%(69)$ & $38 \%(60)$ \\
Level of exercise & 0 & $12.5 \%(20)$ & $69 \%(110)$ \\
General well-being & 0 & $8 \%(13)$ & $73 \%(117)$ \\
The caffeine drunk & $4 \%(7)$ & $57 \%(91)$ & $19 \%(31)$ \\
Alcohol consumption & $5 \%(8)$ & $50 \%(80)$ & $25 \%(40)$ \\
Sugar consumption & $3 \%(5)$ & $49 \%(79)$ & $27.5 \%(44)$ \\
Impact of smoking & $2.5 \%(4)$ & $41 \%(65)$ & $35 \%(56)$ \\
Level of rest and relaxation & $0.5 \%(1)$ & $17 \%(27)$ & $64 \%(102)$ \\
Levels of concentration & 0 & $17.5 \%(28)$ & $64.5 \%(103)$ \\
Balance & $0.5 \%(I)$ & $6 \%(10)$ & $74 \%(118)$ \\
Flexibility & 0 & $6 \%(10)$ & $76 \%(121)$ \\
\hline
\end{tabular}


Table 3.2 Martial arts and health improvements (presented in the order items are listed in the survey)

\begin{tabular}{llll}
\hline & $\begin{array}{l}\text { Less } \\
\text { improvement }\end{array}$ & $\begin{array}{l}\text { Neither more } \\
\text { nor less } \\
\text { improvement }\end{array}$ & $\begin{array}{l}\text { More } \\
\text { improvement }\end{array}$ \\
\hline General health & 0 & $11 \%(18)$ & $68 \%(109)$ \\
Posture & 0 & $11 \%(18)$ & $67.5 \%(108)$ \\
General diet & $2 \%(3)$ & $37 \%(59)$ & $38 \%(61)$ \\
Sleep patterns & $2 \%(3)$ & $46 \%(73)$ & $31 \%(49)$ \\
Level of exercise & 0 & $11 \%(18)$ & $69 \%(110)$ \\
General well-being & 0 & $10 \%(16)$ & $69 \%(111)$ \\
Caffeine consumption & $4 \%(6)$ & $57.5 \%(92)$ & $11 \%(17)$ \\
Alcohol consumption & $4 \%(6)$ & $51 \%(81)$ & $16 \%(25)$ \\
Sugar consumption & $1 \%(2)$ & $51 \%(82)$ & $20 \%(32)$ \\
Impact of smoking & $1 \%(2)$ & $35 \%(56)$ & $17.5 \%(28)$ \\
Level of rest and relaxation & $1 \%(2)$ & $27 \%(41)$ & $52 \%(83)$ \\
Levels of concentration & 0 & $17 \%(27)$ & $60 \%(96)$ \\
Balance & 0 & $7.5 \%(12)$ & $71 \%(114)$ \\
Flexibility & 0 & $8 \%(13)$ & $70 \%(112)$ \\
\hline
\end{tabular}

and posture. Whilst only to a much smaller degree (ranging between 1/3 and $1 / 5)$, respondents noted improvements in smoking, sugar, alcohol and caffeine consumption are also emphasised.

The excerpts from the interviews below highlight the connection between awareness and practice:

...it makes me feel stronger...it's corrected my posture, um... I have scoliosis of the spine which I never knew I had, which is very mild, it's a mild one but you know I kind of lean to the right, so, by doing this standing for these years I've learned how to correct my spine and my posture. Um, and also um...I feel a lot healthier for doing it, my whole body feels better, um.

oh, you know, I was in a queue and I had to wait so I started to do my standing and my balance is getting better, you know I just feel a little bit better, you know, so... the people that stick with it start to get some of the benefits

In considering the levels of health awareness and the perceptions of improvements noted in Tables 3.1 and 3.2, respondents were then asked to comment on how confident they were that any greater awareness and improvements they observed were the result of their martial art. Of the 131 who responded, $90 \%$ said that they were 'very confident,' $8 \%$ 'a little confident' and 2\% 'not very confident.' Therefore, whether the benefits are real or not - and whether any improvements can be attributed in a causal way to their martial 
arts - teachers in this research believe that their practice is making a big difference to their lives and this was clearly important. This theme also emerged from the interview data, as one of the interviewees explained:

I think the first thing immediately was the impact on my joints, because I've got arthritis and clicky hips and so when I started I was quite stiff, because when I was right about 24 to 26 I could barely walk, because my hips were that bad, and so, going on through the years, my hips got worse and worse, so when I started doing karate I was quite stiff, so yeah now that's really helped, and I've got a lot more movement in my hips now, so which loosens everything up. So, physically the obvious benefits were to my joints and my hips and things like that, has really helped, and also just fitness wise. I'm quite fit, although I'm 53 and asthmatic, I'm still quite fit for my age and everything, so fitness wise, brilliant.

\section{Well-being}

Modifying very slightly the British Office for National Statistics standardised national measures for well-being and health, participants in the survey were asked to comment on a number of areas, including the value of martial arts to their life, their levels of anxiety and general satisfaction with life, as a result of their martial art practice. These were scaled 1-10. To illustrate, in response to the question 'since beginning martial arts, are you more or less anxious?' 1 would indicate 'completely anxious' and 10 'not anxious at all.' If we consider 5 as average, and therefore little or no obvious impact, then scores of 7 and above could be considered as significant. However, it is important to remember that responses to these questions are simply from the perspective of the participant and are not intended to indicate anything more than this.

The data from the survey reveals very positive responses. Of those that responded $98 \%(n=136)$ stated that participating in a martial arts provided a sense of purpose to their lives, with $94 \%(n=143)$ believing that it contributed to their satisfaction with life, $88 \%(n=136)$ believing that it contributed to their sense of happiness and $87 \%$ believing that they are much less anxious as a result of their practice. Each of these questions included an open-ended option that prompted respondents to give an example to explain their answer.

I think intellectually mentally, it has helped me focus and I've got a much greater concentration now, whereas before I had no concentration, my head would just drift off, so it has allowed me to be able to concentrate more, so I can think in straight lines, instead of going off on tangents.

Primarily, the responses related to the physical, health and well-being benefits received. However, other aspects emerged as important in influencing the benefits perceived by the teachers. These were very much connected to the 
social and a sense of well-being that results from relationships of trust and friendships that are developed through their practice, as well as that which emerges from a sense of belonging to a network:

...on a personal level I think that the martial arts not only make me healthy but make me feel very confident that I can do things. Um to me learning Tai Chi Chen, is not just about learning a set of exercises, it is about compiling everything that you learn... you learn about physics, you learn about physiology, you learn about psychology, and only when you're able to think of all three and apply them, then you gain the philosophy of Tai Chi Chen, which then allows you to tick everything that you know from martial arts into your own life; to work life and to home life, and actually apply it into different situations.

In relation to the perceived benefits of martial arts to more specific areas connected to well-being, Table 3.3 highlights a range of very positive responses to a number of measures. The data indicate that feeling a useful part of things is clearly a very positive benefit to being a martial arts teacher, whilst help

Table 3.3 The impact on everyday life of participation in martial arts

\begin{tabular}{|c|c|c|c|c|}
\hline & Not at all & $\begin{array}{l}\text { No more } \\
\text { than usual }\end{array}$ & $\begin{array}{l}\text { Rather more } \\
\text { than usual }\end{array}$ & $\begin{array}{l}\text { Much } \\
\text { more than } \\
\text { usual }\end{array}$ \\
\hline $\begin{array}{l}\text { Help with loss of sleep } \\
\text { over worry }\end{array}$ & $5 \%(7)$ & $26 \%(37)$ & $36 \%(52)$ & $33 \%(47)$ \\
\hline $\begin{array}{l}\text { Help with feeling } \\
\text { capable of making } \\
\text { decisions }\end{array}$ & $0 \%(0)$ & $24 \%(34)$ & $42 \%(59)$ & $33 \%(47)$ \\
\hline $\begin{array}{l}\text { Help when feeling under } \\
\text { strain }\end{array}$ & $4 \%(5)$ & $23 \%(32)$ & $38 \%(53)$ & $36 \%(50)$ \\
\hline Help with concentrating & $0 \%$ & $11 \%(15)$ & $41 \%(58)$ & $48 \%(69)$ \\
\hline $\begin{array}{l}\text { Help with feeling you } \\
\text { are a useful part of } \\
\text { things }\end{array}$ & $3 \%(4)$ & $14 \%(20)$ & $35 \%(50)$ & $68 \%(48)$ \\
\hline $\begin{array}{l}\text { Help with being able to } \\
\text { face up to problems }\end{array}$ & $1 \%(I)$ & $15 \%(22)$ & $37 \%(53)$ & $47 \%(66)$ \\
\hline $\begin{array}{l}\text { Help with health and } \\
\text { well-being concerns } \\
\text { in old age }\end{array}$ & 0 & $8 \%(12)$ & $36 \%(5 I)$ & $56 \%(80)$ \\
\hline $\begin{array}{l}\text { Help with the } \\
\text { enjoyment of day to } \\
\text { day activities }\end{array}$ & $1 \%(1)$ & $17 \%(25)$ & $35 \%(49)$ & $47 \%(66)$ \\
\hline $\begin{array}{l}\text { Feeling reasonably } \\
\text { happy, all things } \\
\text { considered }\end{array}$ & 0 & $16 \%(23)$ & $41 \%(59)$ & $43 \%(60)$ \\
\hline
\end{tabular}


with concentration and the general enjoyment of life is also relevant. Finally, a sense that their practice enabled a degree of confidence about their future health and well-being was also interesting suggesting that health for some of the martial arts teachers was not just reactive but also strategic proactivity in planning for their future self.

I think that the biggest personal benefit has been the mind. I really do. You know, it's been a way the discipline and...because everything... everything flows out of that, being able to stabilize my conscious awareness, and of course my fitness and being able to connect with other people that have other similar shared values, but even now, I think it sounds kind of strange, but I think our world, you know, our combined we're having a nervous breakdown on a global scale, but I feel like I am a very still, I have a very solid internal foundation.

I'm almost sixty and I've had a lot of...I've had a lot of, you know, things in my life just like everybody has, so, but you know it's the element of the mental...the mental element has been the element, [that is] not just a stabilized mind but [one that has] the capacity to keep going. In our martial arts practice...they're not just for physical exercise, they are to train this type of resiliency... and that's really it, this resiliency.

\section{Additional benefits}

Alongside the physical, health and well-being benefits that were attributed to martial arts, other positive consequences were noted in the survey and particularly in the interviews. These related much more to the values and way of life that some of the martial arts were seen as providing, both for the teachers and their students. These were largely organised into 'culture,' 'community' and 'inclusion.' The following sections discuss each of these themes, in turn, as seen in Table 3.4 .

Table 3.4 Additional benefits of martial arts practice

\begin{tabular}{ll}
\hline Benefit & Response \\
\hline Regular exercise & $86 \%(138)$ \\
Better self-awareness & $81 \%(129)$ \\
Better balance & $76 \%(122)$ \\
Increased flexibility & $74 \%(119)$ \\
Keeping the brain active & $73 \%(118)$ \\
Feeling part of a club/community & $71 \%(114)$ \\
Greater self-discipline & $71 \%(114)$ \\
Chance to learn & $70 \%(113)$ \\
Meeting new people & $61 \%(98)$ \\
Network of support & $44 \%(71)$ \\
Company & $39 \%(62)$ \\
Structure to week & $30 \%(48)$ \\
\hline
\end{tabular}




\section{Culture}

Culture was an important theme that emerged from the data. In the case of martial arts, culture was related to the norms, values and expected behaviour in and out of training, as the following examples show:

...in martial arts classes [they learn] to be polite, to be polite to each other, to be polite to the teacher...to respect, yeah to respect each other, to respect the teacher

They learn so much about things, and values in society that we've sort of, you know...lost...

...there is etiquette within the dojo. You have to bow before you enter, you have to line up and you have to bow to the sensei, teachers. And so, and it is a show of respect and if they're doing sparing you have to show respect to your partner and things. It is all about that...it's showing respect to your teacher and what you're about to do.

it teaches you some manners, it teaches you etiquette, it teaches you understanding of other people. It's, yeah, it's far more than just a physical thing.

Helping each other featured regularly in interviews too:

Yeah, and everyone helps each other, if they're struggling you help them, you know, and also they'll help you, you know. Um...so it's good, it's a lovely experience.

It's good when, if somebody's getting stuck on something, we will sometimes pair them off with somebody that knows what they're doing and so they're helping each other as well and they're talking about things.

Less frequently mentioned but still important was the value of learning about different cultures to one's own and included language, diet and history:

It is always interesting to learn more of 'foreign' cultures. I find that most students of Japanese martial arts extend their interest into the whole culture and history of Japan.

\section{Community}

Being part of a group and belonging to a particular martial arts community was also mentioned as important. The social connections that were a significant facet of belonging to a group had benefits, not just for the self but also for the people they interacted with in everyday life, as seen below:

So I think just the close proximity and actually being in a group and realising that, oh you can get on with people, people aren't so bad, and 
things like that, it's just, yeah, it does, it has a massive impact on different people in different ways.

I mean there's actually kind of research that shows that doing dancelike more...more synchronized movement together is actually hugely beneficial for people, you know, they do, they have a sense of community, they have a sense that, you know, they get out of isolation. I mean, you know, the fact of the matter is, you know, human beings, you know, we think that we are alone in the world, but we are not we're just a reflection of everything and that because none of us is knitted together though relationships so, the more we can get, you know, in these types of synchronized...relationships that focus not, just on our own, small problems...that we understand kind of immediately that we are a part of the bigger picture. I think that, that that does help them; we come together and we take a class for an hour, or three a week, and then we go out into the rest of our community and then whether we are skilled at the actual form or not, is secondary to whether we start reflecting on that, whether we're driving down the freeway and we let somebody pass us or listen to our spouse or our friends and you know, at the same time I think at least, like I keep looking for a way that, you know, there can be more, you know, type of community...I guess, activism.

However, there appeared a clear distinction to these social connections, which presented as reflective of two types and levels. The first is related to a strong sense of identity and belonging that being part of particular community offered, through connection to a club and its members, either at the local, regional or international level. The second were those more meaningful connections, which are developed over time with training partners and fellow practitioners and are much more associated with friendship. A sense of shared values and interest helps cement these relationships, which are also built on foundations of mutual trust and respect, seen as crucial in martial arts training. These ideas can be clearly seen below:

...but it is nice, we do get them to teach each other there as well, so the ones that, so they're building up a relationship with the other children and likewise in the other, you're building relationships with the adults and the children. They're building relationships with each other. So there is a sense of family and of being this group that comes together.

...being able to connect with other people that have other similar shared values...there's kind of this other thing, they think well there's some way that I'm going to have a sense of connection...

friends for life and er the people I've met when we go training on the winter course, summer course; when I go to different countries the first thing I do is to find out, within in our association anyway, who...what the club is and they'll look after you they'll take you round. 
From a sociological perspective, the following two quotes make an exceedingly interesting observation of what the martial arts community and practice mirror, at least for these two interviewees:

I've made...some very good friends...I've got very good friends who do it, you know. Er... um...it's nice, it's like going to church and just meeting friends there, you know, it's like that, it really is, you know.

It is really...um... to me...it's a bit...it's a bit like religion isn't it really. I'm not a religious person since I was born, I'm really not, some mates will get a lot of pamphlets for their religion, whatever religion it may be and it's a big part of life, it's a constant thread through their life which is fine, um, but I'm not religious, but, Karate has been... I never stopped thinking about it, never stopped...I never stopped practicing really, if it's not physically, it's mentally in my head, you know. And really since the age of eleven, it's just been a constant in my life, you know. I mean relationships come and go, houses come and go, pets come and go, everything comes and goes... yeah, religion it's almost like one.

These ideas will be considered in greater depth in Chapter 6.

\section{Inclusion}

Martial arts were considered to be much more inclusive than other sports, with one teacher summarising this idea beautifully with the statement that 'there's no reserve bench in Karate.' Regardless of physical ability or reputation, the martial arts teachers who commented on this were adamant that their art was open to all. The quote below makes reference to physical inclusion:

[in a discussion of a father who wanted his blind son to learn karate]... and basically it turned out that he'd approached about forty different martial arts clubs and they'd all turned him down. They all said that they would not take a blind person, that you couldn't teach a blind person something like karate, and that if they wanted, if he really wanted them to do it, it would cost him $f^{40}$ an hour. And I said that's crazy, I can't see why a blind person can't do it, and even [one of my instructors], actually said to me, oh I don't think that's a good idea and I said, why not, and so that's my attitude, why not? if somebody turns up and they've only got one leg, we've had people, one legged people doing it, we've had, yeah, we've had cerebral palsy, we've got all sorts, you name it, we will take anybody with any physical or mental disability, we don't mind...we've got someone who's autistic, and we've got quite a few kids who've clearly got something going on, but we don't pry, and we've got a few adults who we think might have various learning difficulties, but again, we don't say anything because nothing to do with us, but you watch them and you see them growing and it's just amazing, just really good. 
The following two quotes are indicative of the broader ways martial arts are seen as inclusive of age and behaviour:

I've had teachers and Principals call me and say, you know, this and this person shouldn't train Karate with you because he's bullying other kids, he's a bad kid...well he's basically a kid who behaves badly, and that resonates with me because that's the message my teachers and my parents gave to other dojos when I wanted to train. And I've been very strict and I've said, well it's my dojo, I appreciate your input but I will teach whoever I please and like we'll recap later and I've yet to have a teacher or a Principal contact me again to say that they've found a student more dangerous.

[it's] a philosophy of inclusion, including people...no matter your skill level, no matter you physical disabilities or er, whatever handicaps you might have, you're welcome to train...people can move along at their own pace, and that to me is perhaps the most magical thing about martial arts because you reach a point...you might love playing football, but if you're not good enough, no one else wants to play with you...now everyone's welcome, everyone can do this, yes you won't necessarily progress as fast as er...as er...some people who have fitness, like eighteen year old, but you will progress, you will improve, it will be better.

These long interview extracts exemplify the different ways that martial arts can include some of those young people who would be otherwise excluded, those with physical and learning disabilities or those considered too challenging to manage other activities and sports. Although anecdotal and not included in the interview, it is heartening to know that the young blind man mentioned earlier is now a class instructor and due to grade for his first Dan black belt in Karate.

\section{Challenges to teaching martial arts}

Whilst the benefits to participating in martial arts, both for the teachers and their students, are considered to be myriad and wide reaching, the teaching of martial arts is not without its challenges. Some of these challenges relate to expectations, the expectations of parent and students, in terms of how they think they can behave, how rapidly they think they will achieve and with respect to whose responsibility the learning actually is. These ideas can be seen below:

the dojo is closed to the parents and there's no windows, there's no way to look in, and you're just have to wait in other parts of the hall, or at the venue...but you're allowed to come in and see the er...the gradings. And um... and that's the difficulty because a lot of parents, they're very used to being heard and seen, when their kids are doing extra-curricular 
activities and er, being barred from doing that I think is hard for a lot of them, so we met quite a lot of opposition there and er, and that I think has been the biggest issue.

The biggest challenge is, is getting them to understand that we don't have a belt system, we don't have a colour system, each one of you progress at a different pace and there is no end to this, so it's a slow process.... lot of people, the younger generations, don't understand that. They come in and they want something right away. They want to use it to fight, they want to use it do something... so the biggest challenge that they...they all want is instant gratification, the other one is that they want to walk out the class feeling no pain.

In addition, the underpinning martial arts culture and the ways that this is taught can be difficult for students who are not used to the style and techniques. In some martial arts, learning through repetition is fundamentally key; yet, this can be very challenging for some students, who are used to a different, less didactic, style of learning. The following examples capture these ideas:

Well, I laugh when I would see people, you know, at um, seminars taking, seriously, taking notes and I'd think, watch him! Look at what he's doing! Absorb it! Don't take notes!

I think the other thing is, teaching what is really a traditional Chinese Art to a Western audience...I mean it comes from a tradition of... watching and copying and repeating, over and over and over again and just this sort of slow grinding out the skill level, so the more you put in the more you get out. It is based very much around repetition and practice which is not always accepted by the Western culture. I think some Western students um...struggle with it sometimes

Learning through experience, or experiential learning, is very much embedded into the practice of many martial arts and these practices, whilst they can be adapted and modified to suit different groups of students, still underpin the ways that many - although, of course, not all - martial arts teachers teach. It is this type of teaching that can be challenging, as seen below:

I teach very much the Chinese way...my teachers...they gave you an exercise, if you didn't understand it, they just left you alone with it...you tell the people to stop thinking, you don't think in this art, you feel!

\section{Value of teaching}

Despite some of these pedagogic challenges, the martial arts teachers were unequivocal as to the value of teaching to them on a personal level. As you might expect, these ranged in scope from a sense of personal development 
that comes from teaching, to the satisfaction that is experienced by supporting and watching the development of others, as seen below:

I am forced all the time to think differently, how am I going to teach... you know I have classes where you have this range like...you know, the twenty-five year old athlete, or the eighty-six year old, you know person with cancer, you have that sixty-five year old with Parkinson's and the person with MS and then you've got the person with macular degeneration, you've got all these people on the floor at the same time, how do you teach all of them something so that they leave in one hour feeling like they've got something? It's really challenging.

The following are examples of some of the open-ended comments included in the survey that link to this theme:

Teaching has given me a feeling of putting back something into the community and passing on skills and knowledge that have been passed down for 400 years.

It enhances my well-being as I enjoy teaching others

It is gift of teaching others important skills

I am more interested in teaching and helping above trying to just make money.

As an instructor I enjoy passing on my teaching skills of karate to others so that they grow in confidence in their own abilities as a person.

I'm a firm believer in that the students teach the teacher as much as being teached [sic]

Martial arts teachers also had clear ideas of what was important in a good martial arts teacher. Table 3.5 indicates the responses to a range of attributes that teachers were asked to indicate in terms of importance or not.

Table 3.5 Attributes considered important in a martial arts teacher

\begin{tabular}{ll}
\hline Attribute & Response \\
\hline Level of skill & $69 \%(111)$ \\
Teaching style & $57.5 \%(92)$ \\
Commitment to own training & $56 \%(89)$ \\
Interest in student learning & $54 \%(86)$ \\
Approachability & $54 \%(86)$ \\
Interest in martial arts & $43 \%(69)$ \\
Sense of humour & $39 \%(63)$ \\
Friendliness & $39 \%(63)$ \\
Authority in class & $23 \%(37)$ \\
Age & $3 \%(5)$ \\
\hline
\end{tabular}


Table 3.5 indicates the importance of skill, style of teaching, training and commitment to the qualities of a martial arts teacher, as opposed to other, more inter-personal-type traits, such as friendliness, authority and approachability. Open-ended comments also suggest that other qualities such as, and among other things, embedding martial art practice into their everyday life, modesty in their actions, manner and behaviour and sincerity in their focus on theirs and their students' development are also key to being a martial artist. Interestingly, when asked whether they considered themselves to be a martial artist, $74 \%$ said 'yes' $(n=92), 14 \%$ 'a little' $(n=17)$ and $13 \%$ 'no or not yet' $(n=16)$.

However, despite the consensus around the instrumental and more affective aspects of being a martial arts teacher, the following quote epitomises much of what can be inferred from the comments and interviews with martial arts teachers as to what a martial artist teacher is:

[What is a martial arts teacher?] I'll be honest, I really have no idea, but as an instructor er...my job is to put out a table filled with all the skills they would need, physically, ethically, spiritually, mentally er, just er, set up a table, let them in and take as much as they can. And I should not be angry if they take just one bite, or if they stay and eat for ever

It is clear that whilst there are some issues and tensions that create challenges for those that teach martial arts, the rewards far outweigh these. Whether becoming a teacher was the result of ambition, their own development or something that simply happened organically, a strong sense of altruism appears to underpin the ways they approach their art, where a recognition that teaching what they have learned is not just a way to earn a living but also something that is intrinsically tied to both the philosophy and the morality of what it means to be a martial artist.

\section{Access to martial arts}

Finally, martial arts teachers were asked to consider the factors that impacted on their ability to teach. Naturally, cost, location and timing were considered as important. However, general misconceptions were also seen as potential barriers, that is, the idea that everyone who does a martial art 'gets thumped' or that Taichi is just for old people. Access to martial arts will be considered in more depth in the following chapters.

\section{Chapter summary}

From the data presented in this chapter, it is clear that for these 160 teachers, martial arts offer a plethora of advantages and benefits; for themselves - which they explicitly attribute to their practice and the development and skills they 
have acquired through it - as well as for those that attend their classes to learn. Whilst their reasons and motivations to learn were as varied as you might expect, the themes of physical fitness, health and well-being were strongly dominant. In addition, the notion of 'improvement' was a central undercurrent, running through all of the data in response to what martial arts had given them - improvements in relation to fitness, health and well-being in particular. And, whilst the friendships, camaraderie and group identity noted were not necessarily presented as an 'improvement' to their lives, it is clear that it enriched and gave a powerfully important meaning to them. How much of this emerges from a sense of belonging and how much could be considered as intrinsically interwoven into all the other benefits that are experienced is not clear. However, it makes intuitive sense to see them as synonymous and mutually dependent, at least in the case of the martial arts teachers. Opportunities for personal and physical development, and on a number of levels, are what give martial arts an advantage and edge over other types of physical activity from the perspective of the teachers. Whilst not without its challenges, its strong ethos of inclusivity and sense of community is one of the key strengths of martial arts for the teachers included in this chapter, and it is also part of the attraction to the innumerable type of students that come to learn to classes around the world to learn. 


\section{Health and physical well-being and the teaching and learning of martial arts}

\section{Introduction}

This chapter considers the remaining respondents in our research, that is, those that were not teachers of martial arts (discussed in Chapter 2). It considers their physical motivations to learn, the perceived health and physical well-being benefits associated with their practice as well as gives 'voice' to some of the older practitioners. The chapter then goes on to focus more specifically on some of the practical factors that also impact on deciding to learn, as well as broader experiences of teaching and learning. The mental health and well-being and social motivations and benefits are discussed separately in Chapter 5 .

\section{Sample}

Of the 515 respondents in the survey, 340 were students of martial arts - in that they did not teach. Fifty-five per cent $(n=187)$ were male and $44 \%$ $(n=151)$ female, predominately in the age range of $36-45$ years $(24 \%$, $n=79)$ and $65+$ years $(23 \%, n=77)$. Again, due to the convenience sampling approach noted in Part 2 of Chapter 1 and in Chapter 3, Tai Chi (53\%, $n=188)$ and Karate $(36 \%, n=123)$ were the martial arts that most of the sample was involved with, although there were a few examples of other martial arts being practised. Thirty-five $(10 \%)$ of the sample had been learning their art for less than a year, a quarter $(n=87)$ for less than 5 years, whilst $16 \%$ $(n=55)$ had been learning for more than 20 years. The geographic spread of the sample was $84 \%(n=263)$ within the United Kingdom (UK) and 16\% $(n=51)$ outside of the UK.

Interviews were carried out with 26 of the survey participants who had volunteered to be included. The interview sample comprised 11 female and 15 male teachers, ranging in age from 21 to $80+$ years. Twenty interviewees were from across England and six were international, including four from the United States of America and two from Norway. Identifiers used and included in this chapter are with respect to gender and age, when over 65 years. 
Identification is limited to these two variables so as to ensure the anonymity of those who took part.

\section{Motivations for taking up a martial art}

As with the martial arts teachers in Chapter 2, we were initially interested in exploring the reasons that underpinned the motivations for people to take up a martial art. As expected, the responses to this were myriad but, for the large part, mirrored those of the teachers. As such, this section explores these physical benefits and focuses on the health and well-being factors connected to health referred to, but in more depth.

\section{Health}

Physical health promoted by, and connected to, fitness was an important motivator for those learning a martial art, more so than for their teachers, with $24 \%(n=85)$ of the sample noting physical factors as the impetus to their decision to learn.

For older people in particular, specific physical issues and concerns with fitness motivated them to find ways they could support and manage their own health needs:

I do have tendon and muscle stretching issues and so to take something that would work with my body - I've got scoliosis so I'm a little bit crooked on one side - to straighten that out, to strengthen those muscles sounded like just the right thing.

Female, 65+ years

Well, in 1997 I was part of a research programme in Oxford for bone density and I turned out to have osteoporosis in my spine, not that I knew it. And osteopenia in my hip and I've been scanned every two years ever since and the deterioration is unbelievably slow. Which means that I was very compressed and I've been able to open my joints and have benefitted hugely and I do not take any medication for the osteoporosis and the consultant here is going along with it, much as it goes against the grain, goes along with my not taking medication because I have the evidence in my scans that the deterioration is remarkable and so that's why I do a lot, relatively speaking ... we don't stop for holidays, and I keep my osteoporosis under control.

Female

It was clear that health and physical concerns were key drivers for many of those who reflected on their reasons for taking up their sport. Below are some illustrative examples: 
[I had] 2 leg injuries, a year apart, with several months of hopping on crutches. It had affected my balance and I wanted to improve it - Male Better balance, when getting on the bus

Female $65+$ years

Most of the older people interviewed were involved with Tai Chi and many made explicit note of the physical benefits of undertaking their art. Some had very serious physical conditions that they were proactively trying to support, often conditions that promoted their initial motivation. The following excerpt highlights this very well. It illustrates how one man, given only three months to live, actively decides to try and find a way to support his health and the recovery he hoped for. Whilst his decision to take up Tai Chi was almost serendipitous, he had a good idea of what he needed to do to support his health - he needed to be active:

Oh, well I had a tumour in 2013; my mobility almost came to zero. I couldn't walk or get in the car, hardly drive the car.... I used to go to the gym three times a week and really watched my weight. But from that time, well, they'd only given me a couple of months[to live].... So I've decided that, yeah, I'm going to get back to my fighting weight ... they warned me about lifting weights and things... after I'd had the treatment on my spine, they said I couldn't do any lifting or carrying, and even carrying shopping bags was not to be encouraged, anything heavier than a bag of potatoes .... So that led me to try something gentle...when I saw a flyer, a Tai Chi flyer, I read it and thought, that's what I need to do. I can't go down the gym ... in many ways it would have been easier just to vegetate on the sofa and say, I don't feel well and all this kind of thing. But no, I think doing Tai Chi, doing something has really helped me, well yes, to become more confident about life generally ... [and] I do feel better.

Male, $65+$ years

However, physical health concerns were not only the motivators of the older person:

I've had problems with my knees for a long, long time just because of using them incorrectly and, and locking them. I used to lock my knees a lot if I was standing up straight for Junior Red Cross or something and you just continue in that way of misusing your body.

Female

Overall though, a general desire to improve physical fitness, stamina, agility and flexibility were the primary physical health motivations, because of the intrinsic ways these areas connect to general health and the connected well-being. 
As noted earlier, physical issues and concerns were important for some of the participants, but specific health conditions were also often noted as relevant to decisions to undertake a martial art. The following list offers examples of some of the health conditions explicitly noted as factors influencing decisions (not listed in any order of importance or emphasis):

- Cancer

- Heart conditions - such as strokes, heart attacks and angina

- Back pains - conditions as well as injuries

- Support for specific health conditions, such as arthritis and Parkinson's

- Weight

Some of the open-ended comments on the survey made reference to the need to support conditions such as asthma, as well as a more general support for things such as pre- and post-viruses and annual colds. The most frequently mentioned comment with regard to health-related motivations, though, was the more general 'health and fitness.' At what point in a person's life their health becomes a focus of interest or prompts their active engagement is not clear in this research. However, whether remedial or preventive, reflexivity and ownership of health appear evident.

\section{General physical well-being}

Broader areas that connect to a more general sense of physical well-being were also noted. Whether to deal with the challenges of daily life perceived to be impacting on health, such as the stress and/or depression that results from significant changes to life, such as redundancy, illnesses or a new baby, or whether looking for something 'a little more' than the mainstream sporting options, all were important in providing an impetus to find 'something,' as the below excerpt highlights:

I took it up basically because I had a three-month-old baby and a threeyear-old and I felt I needed to get out the house. it got to the point where, this was on the Thursday evening and then by the following Monday, Tuesday I would be looking forward to going to the class again because I thought the effects were wearing off ... so I'd notice, I noticed the benefit for several days afterwards and doing a bit of practice in between. So yeah, so then ... it sort of became a bit of a fix but I wanted to go. I think it's the mindfulness side of it that you have to be totally absorbed in what you're doing and [what] you're working on, on reducing the tension in your body ... We've always done a standing meditation as part of the class, and that's been very beneficial. Yeah ... there's been times when I've had quite a lot of stressful things going on in my life since I've been doing Tai Chi ... getting divorced and all those sorts of things, and I've found it helped then, even if I wasn't particularly practicing. I think you 
develop a different sort of attitude to life and you can find that you prioritize things better and know what's important and what you can let go of.

Female

Well, it was curiosity, I'm a person who is very ... aware of my body ... I'm very analytical about it and I like to, for want of a better term, keep fit. I always try to ride my bike rather than get on the bus. I tend to walk rather than go in the car etc, etc, right? Not that I do any organised sport, ever, really because I'm not that kind of person. so Tai Chi seemed appealing just to see what it was like and it was a different kind of thing from what I'd ever done before.

Male

The aforementioned examples offer a particular example of how a recognition of 'needing something,' although not always knowing what is needed, provides the momentum to seek out a focus to support that need. The extracts also show well the self-reflective nature of evaluating benefits. How well the weekly attendance at, for example, a karate class can provide all the benefits and skills hoped for and claimed is not the focus of this study. However, what is both striking and relevant to consider is the perception and belief that it has. Below is just a small selection of some of the comments in this area that are related to how martial arts were perceived to be supporting health:

- It helps calm my physical system; promotes movement that does not strain my joints; and tones my muscles, meditative and centring.

- It is good physical training, where I can train my whole body at the same time, and also [develop]strength, endurance, balance, coordination, flexibility and technique. It is also good for my mind, and it gives me a sense of harmony and peace at mind.

- My balance is better; my posture is better; a bad shoulder is much better and I have a sense of calm.

- My balance and flexibility have improved.

- My personal experience is that it is good for my joints.

- As well as the physical benefits, I find my mindset is more positive.

- Good for confidence plus physical and mental health.

- Physical and mental improvements. Something positive and completely absorbing to focus on.

- Physically and mentally challenging. It offers physical and mental health benefits in terms of strength, flexibility, cardio vascular health, relaxation and a form of meditation.

- Physically and mentally. It is both stimulating and energising. In addition, it is extremely satisfying noticing physical improvements.

- Posture, relaxation and mindfulness.

- Posture, strength and balance, to calm the mind from daily grind. 


\section{Support for health: awareness and benefits}

The survey sought to explore whether participating in a martial art promoted a greater awareness of broader areas connected to health. We were not concerned with how or why greater health awareness develops, only whether it was believed to or not. And, if it did, in what areas? Of the 274 that responded to these questions, $75 \%(n=205)$ stated that they believed they had much more awareness of their general health since beginning their martial arts training, with three quarters $(n=201)$ of those who answered also believing that there had also been improvement in their health. This is, of course, merely personal perspective and no reflection of whether this is accurate or not. It is nonetheless interesting to note that $80 \%(n=220)$ expressed confidence that the improvements they note in relation to both health and health awareness are indeed the result of their martial arts practice and the following examples, from the interviews and survey, offer insights into specific health conditions that are perceived to have benefited from martial arts:

- I've known several people who have had Parkinson's doing Tai Chi and found a lot of benefit.

- I'm asthmatic ... so yes, it helps me.

- I had a sort of sciatica attack and it was very, very powerful for getting rid of that.

- Used to suffer from [being] over-weight and palpitations [but] now lost two stone and issue with heart almost gone completely.

- Ability to maintain a good level of aerobic health and control over asthma and anxiety due to calmness of mind.

- I have mild rheumatoid arthritis which the exercises help. Also, I feel it opens up my airways helping with breathing.

- My oncologist believes in Tai Chi for stress management. I have managed cancer for longer than would be expected.

- Blood pressure and heart rate have considerably dropped since starting karate.

The aforementioned examples are indicative of just some of the numerous examples included in the open-ended comments in the survey. Clearly, whilst we are not suggesting that martial arts have indeed offered the benefits that the respondents noted, there is a value in highlighting the casual associations that they themselves make.

\section{Health awareness}

Martial arts practice, thus, provides opportunities for exposure to aspects of knowledge and practices that were considered as contributing to greater self-awareness with regard to health or, at the very least, promoting an interest that provokes a desire to find out and understand more. 
Table 4.I Martial arts and levels of health awareness (presented in the order items are listed in the survey)

\begin{tabular}{ll}
\hline Awareness of... & Stated more aware \\
\hline Posture & $74 \%(252)$ \\
General diet & $30 \%(103)$ \\
Sleep patterns & $20 \%(68)$ \\
Level of exercise & $68 \%(274)$ \\
The caffeine drunk & $12 \%(4 I)$ \\
Alcohol consumption & $16 \%(54)$ \\
Sugar consumption & $18 \%(62)$ \\
Impact of smoking & $17 \%(60)$ \\
Balance & $73 \%(249)$ \\
Flexibility & $71 \%(240)$ \\
\hline
\end{tabular}

Table 4.1 highlights more general areas where awareness of health-related issues is identified:

As one might expect when engaging in physical activity, much greater levels of improved awareness can be seen in areas such as 'posture,' 'balance,' 'flexibility' and 'general levels of exercise.' However, improved awareness is also noted in relation to 'diet,' 'sleep' and, to a smaller degree, 'smoking, ' alcohol,' 'sugar' and 'caffeine.' An independent t-test also highlights statistically significant differences between men and women regarding levels of awareness in 'general health,' 'diet,' 'alcohol consumption' and 'smoking.' The $t$-test highlights that men report much greater improvements.

Part of the acknowledged changes and improvements in the understanding of personal health could be argued as connecting to greater physical awareness, a result of making links between what one does physically, and how it makes you feel. This is highlighted well below:

...it's more than just a physical thing because you learn about how your body works and how other peoples' bodies work as well.

Male

Wednesday morning I am awake. [because of] my thyroid condition. I often feel a bit dozy but this morning again I was awake. So, something I did last night [in class] has either geed me up or whatever.

Female

I've worked at a desk and desk like situations in totally unergonomic situations, mostly because of the prevalence of laptops over the last 20 years. This place I worked at last, nobody had a desk actually. It's all totally hot desks ... so although there are some desks, very few desks have a monitor 
on them, you're always using your laptop in your lap or on the desk in front of you. So, working for 35 years in computers I've always felt for the last 20 years, my posture was terrible. And so that's generated a lot of tensions in my body and I'm really conscious of those. One of the things that tai chi does for me, and I didn't realise it would do this actually, to be fair, is it's teaching me more about individual muscle control and about proprioception, body awareness and control of where my body is in space ... more aware of my body position and so on ... that's one of the things I've got out of it a lot, is the combination of being able to correct my posture in a way which leaves me feeling great actually ... completely re-energises me, I have to say. ... I do that walking down the road!

Male

...I'd had various operations on my back and I'd gone to what they call the back school, which was learning to deal with it ... they said, tai chi is actually quite a beneficial thing to do because it keeps stretching it. If I have a week off, I can certainly feel the difference. I start to stiffen up. I'm in my 60s now but it makes me notice immediately when I'm not doing it, that I'm deteriorating on my joints and so on so, I have to keep going ... sometimes I have to force myself to go out but when I go I always feel better when I come away ... it takes work and it has to be on a fairly regular basis and as I said, in my own case, I know when my body's deteriorating as far as joints and flexibility go, having said that I can still do the splits! The exercise that you get, it works every part of your body basically so even aerobics doesn't cover the same kind of range of suppleness, stretching, flexibility, the whole of your body is involved at some point and if you stick to it and feel the benefit then you tend to stay with it for years.

Female

The data suggest that the benefits of greater awareness of factors associated with health are wide ranging but are, as might be expected, greater for those who have higher frequency of practice and attendance at class. The relationship between these factors and increased health awareness is also statistically significant $(p=0.05)$.

\section{Health benefits}

Increased awareness is also hopefully connected to practice that directly supports improvements in the areas of interest. Table 4.2 highlights areas where respondents felt that they had experienced change and improvement.

It is heartening to notice in Table 4.2 that increased awareness does appear to result in improvements in the areas that are important to health. Some of these benefits are also statistically significant for different age groups. Again, 
Table 4.2 Martial arts and perceptions of improvements in health (presented in the order items are listed in the survey)

\begin{tabular}{ll}
\hline Improvement in... & $\begin{array}{l}\text { Responses for } \\
\text { improvement }\end{array}$ \\
\hline Posture & $69 \%(234)$ \\
General diet & $25 \%(84)$ \\
Sleep patterns & $20 \%(69)$ \\
Level of exercise & $65 \%(222)$ \\
Caffeine consumption & $9.5 \%(32)$ \\
Alcohol consumption & $9 \%(31)$ \\
Sugar consumption & $13.5 \%(46)$ \\
Smoking & $11 \%(37)$ \\
Balance & $71.5 \%(243)$ \\
Flexibility & $68.5 \%(233)$ \\
\hline
\end{tabular}

ANOVA tests show significant variance in self-reported improvements in the areas of 'general health,' 'general diet,' 'caffeine drunk,' 'sugar consumed' and 'flexibility.' In addition to health awareness, direct improvements were also noted, with improvements in 'diet' $(f=3.62, p=0.001)$, 'sugar consumed' $(f=3.51, p=0.001)$ and 'caffeine drunk' $(f=2.04, p=0.049)$, highlighting some of the strongest variation by different age groups. The open-ended survey data also indicate that 'giving up smoking,' tackling weight' and 'reflecting on alcohol consumption' were key outcomes for a handful of outcomes they self-reported to be the result of engaging in practices that drew greater attention to the physical self.

Whether martial arts were facilitating an opportunity for those that took part to focus more directly on health or not, some of those that responded felt that their martial arts practice helped them manage worry about their health in older age as well as gave tools to manage physical well-being. The following range of examples illustrates this:

A concrete example would be a recent vehicle collision. It was a minor incident on wet roads. We stopped for a pedestrian and I heard the vehicle behind us lock up their brakes. My immediate response was to exhale and relax. It wasn't much of an impact, not even enough to trigger the airbags. That said my wife was sore for some time afterward while I had no soreness whatsoever. In addition to that, staying relaxed during the aftermath was very important. The rush of adrenaline can lead to tempers flaring after such incidents but, my practice certainly has given me the map for how to stay centered even in that turmoil. 
Also, [my] reflexes are quite good.... I [only] discovered recently with one of the grandchildren I can [now] throw a tennis ball into a bucket ... one day I was walking along and, as it happened, I was carrying a glass of wine, don't ask! ... and I tripped and I didn't drop nor spill a drop as they say. So, it has its other advantages.

Female, 65+ years

Well I think also it's quite calming. I have high blood pressure, but I've found that my blood pressure has stabilised quite a lot. Yes, yes. I mean, when my husband died it was very useful really. It really did help me to, sort of, focus, and think outside the box, if you like, and be calm. And I think it's very useful. And also, from a sense of, it's lowering my, I feel, and from the evidence, like my blood pressure is very much more stabilised. But I mean, I know I do take medication as well. And my doctor son would say, well it's because you're taking medication. But I have been taking medication for years and years and years, and not had it as stabilised as it is now.

...when I started, I was actually very ill when I was 59. I had hep C which I'd had for, I travelled a lot when I was young and of course I'd been ill when I was younger. But they hadn't discovered it then, it was a strain of hepatitis and so they told me it was psychosomatic, and it was in my head and it was ... and I was so, I was having such a hard time and I went to a Chinese medicine person and got advice and herbs. And I managed it and stabilised myself, and just thought that was it, and ate differently. And actually, completely changed my lifestyle but, this was in my thirties but when I was, but then when I was 59, I was feeling pretty healthy and I saw that the NHS were offering these health checks because I'd kept away from doctors all over those years. I just lost faith in Western medicine and I went to the doctor and had a health check. I was thinking, "oh this is good, I can maybe get a bit of advice about what to get healthier" and I got a letter from my doctor saying, can you come in urgently, there's something wrong kind of thing and I went in. She said the blood counts are wrong, we need to take some more, and she looked really quite serious. I thought, "oh God", and then they came back and she said, your liver functions are all over the place and you appear to have hepatitis C, I was completely in shock. It was like a ghost coming back to haunt me from the past. And so, she told me what the treatment was which was, I've forgotten the name of it now ... It's just that you had to, the type I had was the most severe type and it was a year's treatment and you had to inject yourself in the stomach once a week and take these tablets every day and there could be severe side effects and stuff. So, I thought, well actually this is pretty nasty. I'd better go for it, I'll give it a try and, by that time of course, I'd trained as a psychotherapist you see. 
So, I had a pretty heavy workload. I was a single parent, she'd just, she'd left home by then, she was, my first grandchild was due. So, I started taking this stuff and I just got worse and worse and developed a wracking cough. And it was horrible and after about five months, I just had to stop work and then that was five months. Once I'd given in ... I was very, very ill and just, and beat the hep C, was off work for 18 months. And then of course when I went back, I was, all my muscles were slack because basically I'd done nothing ... Yeah, I'd been recovering, all my energy had been going into beating hep $\mathrm{C}$ and it was this medicine you see. It's a bit like a kind of, it's a bit like having chemotherapy, that was the effect it had on me because I've got quite a sensitive system. But it did it, we beat it so then, of course, I needed to get my strength and my energy and mind in the world back. And I heard about, my neighbour told me about these qigong classes, I started to go. Absolutely loved it ... [and it has really helped me]

Perhaps somewhat obviously, statistical tests for variance (ANOVA) indicate a statistically significant relationship with some of the perceived benefits of martial arts and frequency of attendance. These tend to be more marked for particular age groups, with those in the age range of $35-55$ years appearing to acquire the greatest levels of perceived benefit. Martial arts and, specifically, physical activity could be considered as providing an opportunity for some of those included to manage risk whilst taking ownership of that potential risk. Given that anxiety connected to risk can be amplified when the risks are unclear and when accompanied by a sense of powerlessness, this is positive on a number of levels. The following quotes highlight well these ideas:

I've had problems with my knees for a long, long time just because of using them incorrectly and, and locking them. I used to lock my knees a lot if I was standing up straight for Junior Red Cross ... and you just continue in that way, of misusing your body. I wanted to do something that was actually mobility based ... to improve balance and flexibility ... and prevent self-injury as well.

Female

...[it]gives us a bit of [reassurance] ... I certainly think about it [my posture] more or less all the time really. If you're moving around or making sure that you're sitting comfortably but correctly.

Male

...it's really good for balance and using your body correctly, standing and moving correctly. So ... you're going to have less pain, or no pain.

Female, 65+ year 
But I think you do need to exercise. I mean, I remember my mother at my age, and she was old, old! She couldn't do anything. And I was fully determined that I wasn't going to be like that. And I think that if you don't use it you lose it!

Female, 65+ years

...it makes me feel stronger ... it's corrected my posture, um ... I have scoliosis of the spine which I never knew I had, which is very mild, it's a mild one but you know I kind of lean to the right, so, by doing this standing for these years I've learned how to correct my spine and my posture. Um, and also um.... I feel a lot healthier for doing it, my whole body feels better, um...

it is very good for our health. I have hip and knee problems, probably the start of arthritis and certainly moving in the way you do for Tai Chi keeps everything ... though it occasionally hurts.

It's again a visualisation technique which you can do lying down and basically what you do is you breath the Qi energy, because apparently, supposedly, it moves through your bones and your tendons and you actually sort of use your breath to breathe the Qi up one leg and down the other one and then back up to your arms.... I had a sort of sciatica attack and it was very, very powerful for getting rid of that and just clears the chi in your bones. But you really notice, you know.

In many ways, the health benefits articulated as directly or indirectly resulting from involvement with martial arts could be seen as correlating with a greater sense of health ownership, whereby the taking of responsibility for one's own health - either because the initial impetus for taking it up was a recognition that something needed to be done to support health or via the process of reflecting on the connection between physical well-being and physical practices. In many ways, a lot of the confidence expressed could be argued as emerging from a sense of 'taking control.' For one survey respondent, martial arts were 'an investment in old age.' Arguably, they are also an investment in self, health and general well-being. The examples below capture some of these ideas:

[discussing younger students in the group] if it comes down to energy, I obviously can't outdo them. They have much more energy than I do, but I would say for the sheer flexibility of your body, fitness, my stamina level is terrible but that's because I've got heart problems and stuff now. 
But to give you an overall picture without a shadow of a doubt it's, and I think it also, it keeps your brain active. I've also unfortunately suffered a stroke... But yeah, you'd think the old brain would start to go in to remission, but it hasn't. I still remember all the stuff and I still learn new stuff. I'm still an active musician funnily enough and I remember all that still. The simple coordinating facilities that martial arts, that you have to do, you get this with tai chi, you have to remember the form don't you [it all helps]?

I think the first thing immediately was the impact on my joints, because I've got arthritis and clicky hips and so when I started I was quite stiff, because when I was right about 24 to 26 I could barely walk, because my hips were that bad, and so, going on through the years, my hips got worse and worse, so when I started doing karate I was quite stiff, so yeah now that's really helped, and I've got a lot more movement in my hips now, so which loosens everything up. So physically the obvious benefits were to my joints and my hips and things like that, has really helped, and also just fitness wise. I'm quite fit, although I'm 53 and asthmatic, I'm still quite fit for my age and everything, so fitness wise, brilliant. I think intellectually mentally, it has helped me focus and I've got a much greater concentration now, whereas before I had no concentration, my head would just drift off, so it has allowed me to be able to concentrate more, so I can think in straight lines, instead of going off on tangents, because I've got quite.... I've got quite a chaotic way of thinking, so I have to train myself to thinking in sequences, so I could remember sequences, because obviously doing line work you have to remember sequences of up to ten moves.

\section{Teaching and learning}

Reasons for beginning a martial art are myriad and some of these have already been discussed. However, alongside health and physical well-being and mental well-being (discussed in Chapter 5), other factors were also noted by some of the participants. These included responses across the themes below:

- Children are learning

- A desire to learn something

- A desire to make social connections

Practical considerations were also relevant and considered important when deciding to undertake martial arts. Respondents were offered 13 items they could select from, including things such as 'cost of classes,' 'experience of the 
teacher,' 'getting to classes' and 'time.' A Cronbach's alpha test of internal reliability showed that the questions used were appropriately reliable, with a reliability level of $a=0.818$. Considering all of the options offered, participants were asked to list their three most important considerations when deciding where to go and who to train with. The 'skill of the teacher,' 'experience of the teacher' and 'getting to class' were the items most frequently selected. Interestingly, these factors were also factors identified as most important by the martial arts teachers. Perhaps somewhat surprisingly, instrumental factors such as cost of classes, clothing and equipment were not of primary importance. Clearly though, the teacher is key, in terms of their expertise and ability to teach.

The benefits associated with learning were numerous and encompassed physical, mental and social domains. Below is a selection of comments from the survey that made reference to learning benefits, although this was not directly asked within the survey:

- I believe that learning martial arts has improved my life by increasing self-discipline and increased awareness, and confidence.

- I like having a 'practice' that can evolve throughout my life.

- I stay in shape physically, it makes me more harmonious mentally, and it also gives me self-confidence and a sense of pride over what I have learned and can achieve.

- $\quad$ Satisfied - [I] like the learning aspects, [the] enjoyment of courses that offer additional skills, for example, self-defence (knives, etc.) enjoy combat, enjoy variety, friendship, something to do, enjoyment, particularly good for short-term memory!, satisfaction of being graded, interest in the way the body can move to create 'effect' unsatisfied as think flexibility could be improved faster, realisation that emphasis tends to be focused on junior grades (based on numbers), feeling some aspects can get a little repetitive.

- It gives me strength to overcome the symptoms of my medical condition and [having a] focus helps with that too. The learning aspects keep my brain from becoming stagnant. It's fun, relaxing and strengthening.

- Consistent learning in a group is very enjoyable and never dull. It carries over into everyday life.

- Training in martial arts gives the ability to maintain or improve fitness and agility. It also allows you to discuss methods with other participants and gives you the confidence to put forward individual opinions. It also helps to keep you engaged as the more you learn, the more questions arise. This helps with thinking, mental agility and the opportunity to put forward these questions to your colleagues and instructors.

- Learning and practising Taiji has given me lots of self-confidence. Initially, I thought I would not be able to learn the basic 18 form. I exercised and practised hard and after six to seven months Shifu told me I had 
started to look like I knew what I was doing. I am moving on to 74 form, old style Ylu and taking sword also. I just love being able to accomplish all that I have done!

\section{Learning and the martial arts teacher}

Using a traditional, taken for granted definition of what learning is, we can agree that a key requisite for learning is a teacher. A teacher shares knowledge, demonstrates, corrects and encourages. Teaching within a martial arts context is also physical and communal (in most cases). Data show that the martial arts teacher is important to the decision to start martial art training as well as to stay learning. Table 4.3 lists the 11 attributes considered most important in a teacher from survey response. Largely, the attributes listed can be considered as capturing three key dimensions: personal ('friendliness,' 'approachability,' 'sense of humour' and 'interest in student learning'), professional ('teaching style,' 'authority in class,' 'level of skill,' 'commitment to personal training' and 'interest in martial arts') and demographic (age and gender):

Figure 4.1 graphically illustrates the importance of professional skills over the personal qualities that could be considered important in social interactions. The relative indifference given to demographic factors such as age and gender reflects the priority given to the social and acquired status of teachers' knowledge and character, rather than those prescribed. Although not a feature of this research, such a finding appears to suggest that martial arts are potentially offering a more level playing field in relation to social equality in sport, where it is skill and experience and not age and gender that matter. Interestingly, there were no real differences within the survey responses in relation to the importance of different attributes, aside from men rating 'interest in martial arts' significantly higher than women, whilst women valued a 'sense of humour' significantly more than men.

Table 4.3 Attributes considered important in a martial arts teacher

\begin{tabular}{ll}
\hline Attribute & Response \\
\hline Level of skill & $69 \%(111)$ \\
Teaching style & $57.5 \%(92)$ \\
Commitment to own training & $56 \%(89)$ \\
Interest in student learning & $54 \%(86)$ \\
Approachability & $54 \%(86)$ \\
Interest in martial arts & $43 \%(69)$ \\
Gender & \\
Sense of humour & $39 \%(63)$ \\
Friendliness & $39 \%(63)$ \\
Authority in class & $23 \%(37)$ \\
Age & $3 \%(5)$ \\
\hline
\end{tabular}




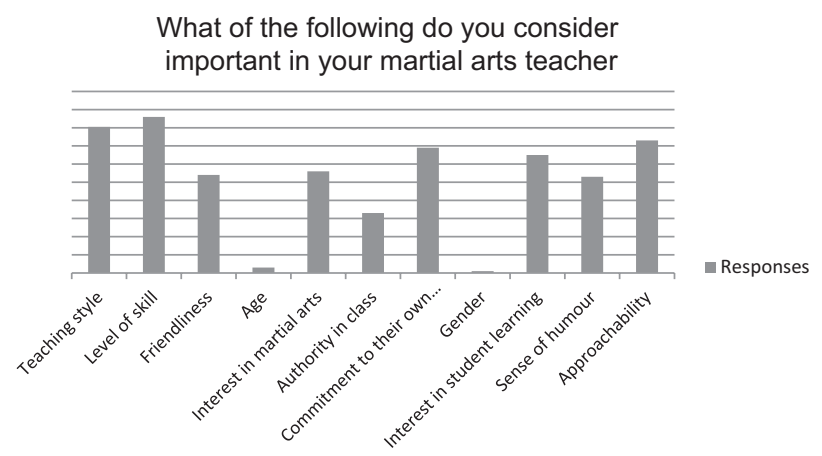

Figure 4.I Graph showing attributes considered important in a martial arts teacher.

Interesting, whilst the teacher is considered important, it was skill rather than personality - to some degree - that appeared relevant. This can be observed in the following extracts:

... [one of my teachers] is very, very special and has a wonderful manner about her ... she doesn't, how can I put it? She's not didactic but, she explains why she does certain moves for something ... this is why I find it frustrating with my younger teacher, that he doesn't see the importance of learning where your exercise is coming from.

Female

I'm not independent, I'm dependent upon the class for regular, a weekly regular practice. [My previous teacher] had created an extraordinary social network where you felt really welcome. It's not unwelcoming in [my new] one, but people ... there isn't that ethos, they are welcoming, but there isn't that ethos connected with the club ... [so], the teacher is important, I think. The trouble is it also means that they're fairly narcissistic, they've got to, that's a real problem, I've been going to classes and I'm saying that these people are somewhat egomaniacal, [but] had they not been, I wouldn't have learnt tai chi at all.

$$
\text { Male, } 65+\text { years }
$$

\section{Qualities in the teacher}

As noted earlier, knowledge and commitment to their own training, as opposed to personal characteristics, were considered as the key qualities, important in a martial arts teacher. Inferred from this it that, from the perspective of their 
students, being an authentic martial artist was more important than being, say, humorous or friendly. However, that is not to dismiss the importance of finding the 'right' teacher or one that could 'teach.' Finding a teacher who was trusted and with whom there was 'rapport' was considered important, but arguably, not as important as their skill in teaching. For some, this was considered more important than the martial art itself. Evidencing their own understanding of their art as well as an ability to share and teach was an important characteristic and quality of a good martial arts teacher.

Naturally, skills in communication were also relevant. Alongside an ability to acquire and then disseminate knowledge of their martial art, encouragement and fairness were seen as important. The status given to the role of the teacher, therefore, highlights well a social dimension to learning, where social interactions and notions of 'teacher and taught' are significant to the practice, understanding and motivation to do both of these things. This is illustrated well in the following examples:

Going to classes is important as well as individual practice, because of the shared energy of the group. To a martial artist this is a real thing.

(survey)

Finding the right martial art ... I think certain arts fit certain personalities. I'd probably say I judge the teacher by his/her students rather than their own skill. It's one thing to be a good martial artist, but a skilled martial artist may not be able to teach very well.

(survey)

Whilst the teacher was considered important, how best to find both a class and a good teacher was not easy; indeed, it was potentially very challenging for some. The following comment from the survey highlights this issue:

Finding a teacher/class/session if a right pain in the bum - the best way being recommendation of course. There seems to be no easy means of finding out anything definitive, there is no register, no standard, no overarching sports body. In fact, the different styles, splinter groups and codes are somewhat baffling ... all rather confusing.

(survey)

\section{Challenges to learning}

In addition to exploring the considerations important in deciding to take up a martial art, it was also important to consider what the key obstacles to maintaining the training were. The same 13 items that were considered in relation to decision-making were repeated and again, including things such 
as 'cost of classes,' 'experience of the teacher,' 'getting to classes' and 'time the classes started.' A factor analysis was carried out on all of the options, with a factor cut off point set at 0.5. In short, factor analysis assesses variability amongst correlated variables. Considering barriers that impact on ability to attend classes, the calculated eigen values suggested that five components explained $81 \%$ of the variation $(25 \%, 19 \%, 18 \%, 11 \%$ and $8 \%$, respectively). The five were then reduced to three that were considered most significant and cumulatively explained $62 \%$ of the variation $(a=0.743)$ : (1) practical - including costs, start time and length of class; (2) intrinsic - including encouragement from others and support from family and (3) professional - skill and experience of the teacher. In short, we can summarise the factors that are most correlated with challenges to learning, that is, the factors that are most directly connected to discontinuation of training below:

1 Time and cost

2 Moral support

3 Teacher

Whilst it is logical that if the teacher is important in deciding to learn a martial art, then they will also be an important influencing factor that impacts on a lack of desire and motivation to continue; that is, they become a barrier to engagement. However, it is interesting to note the importance of encouragement and support to maintaining momentum from others, including family and friends. This suggests that positive reinforcement remains a key teaching aid, regardless of age.

\section{Being a martial artist}

Finally, participants in the survey were asked to write, in their own words, what they considered a martial artist to be. From the $211(62 \%)$ who responded, a range of qualities were highlighted. The word cloud in Figure 4.2 highlights the range, as well as the repetition, of the words that were used, where the bigger and darker the word, the more frequently it appeared:

We can see that the key qualities considered important to a 'martial artist' were related to their martial skill, their commitment to their training, knowledge, physicality and confidence.

Two hundred and sixty-six participants replied to the questions which asked them whether they considered themselves to be a martial artist or not. Rather hearteningly, 63\% $(n=162)$ said 'yes/a bit,' 19\% $(n=51)$ 'no' and 18\% $(n=46)$ 'not yet.' Within these responses, there were no real differences by age or gender, aside from the fact that the greater proportion of those indicating 'no' were aged 65+ years. As might be expected, the number of years that one had been training for was also significant $(p=0.05)$ in predicting who would be identified as a martial artist. 
control confident attitude Eastern good ability individual experience Someone practises martial oneself knowledge movements practises martial art able use Someone practices martial applications dedicated lives body mind Anyone improve best ability committed techniques form system life self confidence trains exercise practices martial arts able skilled help self focused fighting self defence learning apply martial arts $_{\text {aware }}$ person ${ }_{\text {mind }}$ Someone

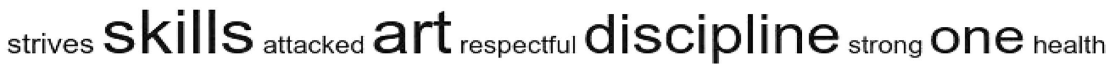
practices hard way someone practises body way life Someone practices traditional use self defense physical teacher

martial artist well develop healthy trains martial arts benefit martial artist someone important others regular understanding physical mental interested wants know think teach time balanced Someone trains

Figure 4.2 Word cloud of key words used to describe a martial artist.

\section{Chapter summary}

Data presented in this chapter have highlighted the various ways that health is a very present factor for many in their martial arts. Health appears to influence and motivate the commencement of practice, or it is directly impacted. Impact, for the purposes of this research, is something that is very much perceived. However, whether it was because of increasing awareness, support for particular conditions or with improvements in specific areas, for many of the respondents in this research, martial arts have had very positive benefits for their health. The data also offer some interesting windows into the importance of reflexivity and the ownership of health, that is, the recognition of the connection between what we do and how we feel. Thus, whether someone is coming towards martial arts because they are seeking to proactively support a particular need they have identified or the motivation is remedial, managing and supporting one's own health is clearly significant to the perception that what they are doing is helpful. This chapter also highlights the importance of the teacher in this process, recognising that their skill, knowledge and expertise are crucial for motivation to begin, and then stay, training. 


\section{Chapter 5}

\section{Well-being}

\section{Introduction}

This chapter presents the data from the students of martial arts but focuses specifically on well-being. Well-being is a particularly important aspect of general physical health as it interconnects so directly. Feeling well and a sense of physical well-being are the important features of how we feel about our lives more generally, alongside how we feel about, and manage, our everyday lives. In the context of this research, we draw on ideas of well-being as defined by MIND (a British mental health charity). Thus, in this chapter, mental well-being refers to the subjective aspects of self-confidence, positive self-esteem, ability to live and work productively, engagement with the world and an ability to cope with the stresses of everyday life (www.mind.org.uk/ information-support/tips-for-everyday-living/wellbeing/\#.XGq_2i2cYRU accessed 2018). Mental health is also touched on but only very briefly.

\section{Sample}

This chapter includes the data from those discussed in Chapter 4, to recap a sample of 340 from the total sample of 515, all of whom were students of martial arts: $55 \%(n=187)$ male and $44 \%(n=151)$ female, predominately in the age range of $36-45$ years $(24 \%, n=79)$ and $65+$ years $(23 \%, n=77)$. The geographic spread of the sample was $84 \%(n=263)$ within the United Kingdom $(\mathrm{UK})$ and 16\% $(n=51)$ outside of the UK.

Twenty-six interviews were conducted with the survey participants - for more information, please refer to Chapter 4.

\section{Martial arts and well-being}

We slightly modified British government measures for well-being and anxiety, to place the focus for these items on martial arts. These measures had an internal consistency of $a=781$. The questions asked participants to consider, since starting martial arts, how much more or less improvement they 
Table 5.I Improvements in well-being since beginning martial arts

\begin{tabular}{ll}
\hline Item & $\mathbf{7}$ or more \\
\hline Feel life is more worthwhile & $97 \%(n=286)$ \\
Satisfied with life & $84 \%(n=244)$ \\
Happier with life & $82 \%(n=232)$ \\
Less anxious & $75 \%(n=212)$ \\
\hline
\end{tabular}

felt in the areas of 'feeling that life was worthwhile,' 'feeling satisfied with life,' 'feeling happier with life' and 'feeling anxious.' These questions were presented as a scale from 1 to 10, with 1 being 'much less,' 5 'about the same' and 10 being 'much more.' Using 7 as an indicator that some improvement had been noted and attributed to martial arts from the perspective of the respondents, we can see from Table 5.1 that for the 295 that responded to these questions, overwhelming improvements were noted:

It is also interesting to note that an independent $t$-test shows that there is no significant difference between men or women on these measures.

The open-ended comments from the survey highlight a range of factors that offer insights into the ways that the practice of martial arts is seen to contribute to these outcomes. These are loosely organised into key themes and are summarised below:

- Confidence - general confidence or confidence emerging through the development of physical self, via greater self-discipline and focus, skill set to support everyday life and pride in everyday achievements.

- Intellectual - greater understanding of self and own abilities, learning and growth as a person, moral development, that is, understanding of right and wrong, maturity and offering 'a way of life.'

- Social - network of support, friendships, community belonging and social happiness.

- Management of stress and the link to health and well-being support for managing and dealing with stress and the subsequent support for health through practice as well as the greater confidence resulting from a sense of self-efficacy and control.

It is important to make clear that not everyone who participated in martial arts felt so positive and, where they did not, it was usually attributed to other factors that were taking precedence in their lives at that moment. One respondent made an interesting observation in that for her, martial arts had made her much more aware of how she was feeling and as a consequence, she felt a lot less happy and was much more anxious. However, she saw this as 
a positive thing, in that this awareness had highlighted the pressure she was living under and the changes she needed to make in her life.

Interestingly, the notion that martial arts allow 'ownership' of health and well-being is very evident once again. Indeed, two participants who noted that they suffered with clinical depression expressed the idea very clearly that martial arts had empowered them, as can be seen in the following comments from the survey:

It's the main thing that has actually helped me maintain my Mental Health, rather than things I've been involuntarily subjected to.

It has improved both my physical and mental health. I find it easier now to go into a meditative state when I find I have things I need to address.

My mental health has dramatically improved.

I suffer from depression and social phobia and taichi has helped me live more in the moment

\section{Well-being and confidence}

Confidence was an important aspect of both the survey and interview data and was referred to either explicitly or indirectly. Confidence in martial arts is not straightforward, however, and can relate to that which results from physical development, the development of discipline, meeting new people and learning something new. It can also be a combination of any of these. Therefore, benefits in these areas are not isolated but are fluid and interconnected. To illustrate, greater physical confidence can lead to greater discipline, which, in turn, then links to greater self-confidence. The direction of these relationships is also myriad. Therefore, whilst we present the data here under discrete headings, it is important to keep in mind the complexity of this area. Table 5.2 also offers a useful point of reference.

Physical confidence: Physical confidence appears to be a positive consequence for a number of people and in a variety of ways. For example, physical self-assurance was directly connected to mastery of the various arts and their

Table 5.2 Other benefits to martial arts practice

\begin{tabular}{ll}
\hline Benefit & Responses \\
\hline Better self-awareness & $81 \%(129)$ \\
Keeping the brain active & $73 \%(118)$ \\
Feeling part of a club/community & $71 \%(114)$ \\
Greater self-discipline & $71 \%(114)$ \\
Chance to learn & $70 \%(113)$ \\
Meeting new people & $61 \%(98)$ \\
Network of support & $44 \%(71)$ \\
Company & $39 \%(62)$ \\
Structure to week & $30 \%(48)$ \\
\hline
\end{tabular}


connected forms, by developing strength, stamina and ability. However, confidence was also more nuanced for some, and directly related to a sense of the physical self, as the following quote illustrates:

We've actually got a lady who trains with us on a regular basis and she's $4 \mathrm{ft}$ 6in ... she's now teaching these adolescent kids who are towering over her and intimidating but you've got the confidence to stand there and go, yeah, do what you're told Male

Discipline and control over the physical self can be very empowering for those who may feel less confident because they are small, feel weak or are shy:

...I suppose I am probably more confident than I was because I can stand fairly tall, I'm only short, but I stand as tall as I can. I'm not frightened to go out on my own...I suspect doing the karate has allowed me to stand up in front of people.

Female

... as a girl, or as a woman, it gives you a confidence that even if you don't, like, you don't walk on the streets and think that you're going to [get in a fight] but it gives you that confidence that you just carry with you, in yourself and that helps you in your everyday life as well I think. Female

I can give you an example with my son actually, he was a very, very shy little boy and [physically very awkward]. When he started doing karate...he realised he was good at it, his attitude changed completely, he was no longer shy with anybody, he would do anything, go anywhere. So that is a positive effect, just the fact that he knows now that if he ever got into a situation where he was threatened, his confidence that he can get out of it rather than get involved in it is such that he'll walk away.

Male

In these examples, it could be argued that the physical, external body is an important expression of the self. And, if this is so, then it is also reasonable to assume that if there is little or no confidence in the physical body, then a person is at risk of experiencing a lack of confidence in other aspects of their life, as well as lower self-esteem?

Discipline: Being focused and disciplined in training is also important for well-being, and it could be argued that this is because well-being is a consequence of a sense of self-satisfaction that results from doing something that takes an effort. This can be seen in the following examples:

...it does help me because the very fact I make the effort to go out there twice a week to do it, yeah, it [does] get too much for me, but I [still] make the effort to go along.

Yeah, I think...you learn lots about yourself and you learn to practice hard, to never give up. You learn about self-discipline because you have 
to train really hard and be focussed for a long period of time in order to obtain a new grade or to learn new things - really, that you can't give up and I think that's a good lesson that you can transfer to other areas of life as well.

Female

...the point about it is, with karate, people don't realise, it's not [just] physical, it's more, it can be more mental than physical, and you've got to remember things and go through things, and it's a mind job...people think, oh it's just a matter of doing some exercises and learning these techniques, and one thing or another, and that's it. You feel great, you come home. It's not that at all, it isn't that.

Male

However, discipline also translates to other aspects of life, most notably those things that require perseverance and commitment, such as completing tasks or doing things that are stressful and tiring. A sense of accomplishment is also a really important outcome and is also linked to discipline and effort. However, the more explicit links to mental well-being can be observed in the connections made between mental discipline and the relief of stress with greater self-awareness and confidence, primarily because martial arts provide a focus. Focus in the context of martial arts is linked to a focus on goals, physical self and/or pain and stress management with a focus on the present, as seen in ideas connected to 'mindfulness.' These ideas are exemplified in the interview excerpt below:

...it does, it teaches, it calms you down, it teaches you some manners, it teaches you etiquette, it teaches you understanding of other people. It's, yeah, it's far more than just a physical thing. I think the physical part of it only a fraction, I think the mental and emotional part is much more.... I think people's personalities really come alive and be transformed by doing it, it's quite a powerful thing to do as in, character building, so I think that's the biggest thing. It's not so much necessarily fitness, it's not necessarily things like that. It's not necessarily a physical aspect, but it's the mental aspect that it has on people, which is far more powerful.

...I think for me, and what's interesting about this Goju club...it's that traditional element of the blend of respect for teacher, respect for self, of physical improvement, also mental improvement, and they really do go together. And yes, you can get that from jogging, but you don't get it in a spiritual sense, or it hasn't got the roots in philosophy in the way that the martial arts can have if you find the right martial arts club. 
Martial arts is not only for fitness but teaches many valuable lessons in life including respect, self-confidence, self-motivation and discipline.

\section{Intellectual}

Interestingly, many of the participants made references to the intellectual benefits of their martial arts. In this instance, 'intellectual' refers to the development of reasoning and understanding and, for some in this research, was very much connected to a far deeper understanding of themselves and their own abilities. As can be observed in Table 5.2, of those that responded, 'keeping the brain active' was important to $73 \%(n=11)$, whilst having an 'opportunity to learn' was important to $70 \%$ (113). The type of intellectual learning varied but most often related to things such as growth as a person, primarily through a perceived improvement in maturity, and a sense of a greater moral understanding, for example, a greater understanding of right and wrong. These can be relatively simple things connected to perseverance and diligence in everyday life or connected to how to respond to situations and circumstances in a balanced way. For many, martial arts were, therefore, seen as offering 'a way of life,' either through the philosophical dimensions that underpin most of traditional schools of martial arts or, simply, by applying what was learned in the dojo (a space in which martial arts are practised) to everyday life. The following extracts from interviews are offered to illuminate some of these areas:

\section{Learning}

the point about it is with karate, people don't realise, it's not physical as, it, it's just as much, it's more, it can be more mental than physical, and you've got to remember things and go through things, and it's a mind job...people, people think, oh it's just a matter of doing some exercises and learning these techniques, and one thing or another, and that's it. You feel great, you come home. It's not that at all, it isn't that. You get, you start looking a bit deeper

Yeah, so of all the things that I've done in my life I feel that this is the most important, I continually study, and I actually was very interested in different religions from a sociological point of view, and so I can tie these up to what I've learnt. I can see where the exercise has come in and... when you study things such as tai chi, qi gong, it takes you down other paths, you learn about history, you learn about all sorts of different things 
I'm a psychotherapist [and we are always] talking about personality type rather than...where we come from, that early connect, there was something that drew me, yeah. The East and the eastern philosophies and, from a very early age, and even actually in recent months watching Monkey, you remember the programme Monkey?...that is so deeply immersed in Buddhist philosophy, which as a kid you don't really pick up, but as an adult you really watch it and you think, my God, they're talking all about the scriptures and about compassion and about this, that and the other, but at some level you must absorb that

...sometimes I think it should be compulsory It absolutely should definitely be on the NHS. I went to my doctor and about something unrelated and I mentioned that I did Tai Chi and she said what's that? So, there are still people in the medical profession that don't have a clue. I think teaching, well in China of course they do learn it in the schools. And they do it from a very young age but it's a bit difficult to get through to our youngsters.

Yeah absolutely and it also has a lot to do with being schooled, being taught and that's something we lose when we're older because we're not at school anymore and I like that feeling of being instructed and led and feeling like you're all doing something together. It's great.

Through my study of Taekwondo I have learned 5 different tenets; Courtesy, Integrity, Perseverance, Self-control, Indomitable spirit. By living my life by these tenets, it has led to me on a path to a more satisfying life. (survey response)

For some, their learning through martial arts contradicted with their more traditional learning quite strikingly, and raised questions connected to the 'culture of learning':

I do actually think that the Chinese medical view of fluids as energy, as a kind of third fluid flowing around the body is actually a very sound one. It's just a pity that Western medicine seems to have been predicated on cutting up dead bodies ... Chinese medicine is more based on a dynamic system I feel. That's it...my degree is in chemistry. And I had a, my great friend who was my tutorial partner at university was a, an SRS and I mean she was just so, well she was extremely kind of what shall I say? Dismissive of anything like that. It's, well it's not a scientific mind, it's a closed mind actually in that. Yeah, I think Einstein was quite keen 
on keeping, people keeping their minds open to everything, you know. I think there's been a, the debunking attitude that scientists have had towards all sorts of alternative things from religion to homeopathy[and it] has not done anyone any service at all apart from the big pharmacological companies, dare I say it.

\section{Culture and ethics}

Numerous comments in both the interviews and the survey made reference to the culture of martial arts, the philosophy and the ethics that were perceived to underpin the practice. It is important to note that this was not important for all, but the frequency of references suggests that it was relevant for a significant number. The following comments from the survey are illustrative of some of these:

- The mental challenge of understanding a complex system born of a different culture. I'm a lot more interested in Chinese culture, history and medicine theory. This affects the books I read, TV programmes I watch and articles I read on Wikipedia.

- The realisation that this type of exercise training has actual medical benefits too and has been around for a long, long time.

- Aside from the health benefits outlined earlier, martial art is a rewarding lifelong practice which can help with all aspects of life. It offers a philosophy to live by, goals to aspire to and friendship and camaraderie.

- I had several years where I stopped training and it was a difficult gap to fill. Tai Chi and traditional martial arts cover a huge breadth of knowledge from the simple physical training and fitness aspects through to the meditation and philosophy. Very difficult to find all that in one place in any other hobby/activity, particularly of western origin.

Ethics or at least a framework by which to live one's life was also an important dimension:

I think the more traditional, the more ethically based martial arts are very much connected with that sense of moral ethical way of life. So yes, of course, and it's a great paradox as often happens with eastern religions, through acceptance of training how effectively to kill people, you give up violence...you realise that there's always a healthy respect for others...I'm very intrigued in that paradoxical element of Eastern philosophy...so it's a part of my life and always has been and hopefully always will be. The way I am as a martial artist and the way I am outside of the dojo, are one and the same thing, and they're something that you carry with you...there's a connection with nature that I think fits well with the martial arts and those resources are highly important to me, so in terms 
of sense of wellbeing,...if I'm not doing one of those things on some sort of [regular] basis, then it does impact on both me physically but also mentally as well. that traditional sense is disappearing, that connection with the spiritual, with, that it's still recognised as good for health, but I think there's something about, and maybe this is a broader topic, but around the way that society has developed that some of those traditional values are disappearing, attention spans of kids and ... the essence of course of martial arts and mindfulness is about being present and the majority of how we have to live our lives now disconnects us from that because presence comes through a screen of some description rather than through the breath...but it is an observation

Examples of some of the responses from the survey are given below:

- I have learned so much - from telling the difference between my right and left to understanding more what is right and wrong.

- Martial art is a way of life for me now not just something I do as a hobby and it feels good to challenge yourself every time in the lesson to improve yourself as a whole.

- Martial arts led me to Buddhism, Taoism and mindfulness meditation. This has ultimately led to an in-depth exploration of myself and retraining as a mindfulness-based psychotherapist. The arts give me a resource and resilience in this difficult territory whilst providing me with a way to stay healthy and strong.

- Over the years, it has given me physical health benefits as well as mental health benefits, and has allowed exploration of philosophical thought associated with martial arts and Buddhism/Taoism.

The data highlight the importance of having a multi-layered learning experience. For some, learning martial arts is more than just the acquisitions of a set of moves; it is also structured by rules and codes of conduct. Access to ideas, which are arguably deeply entrenched in the ways of life for millions in the East, also offers an even deeper level of learning for those that seek it. Interestingly, whilst there are no real differences in the aspired to learning gains, there are some differences in the focus for the learning, where the social and community aspects of learning appeared to be more significant for women, but the more individualised approach was more important to men. What makes this interesting is that clearly learning martial arts were able to cater to the various needs of all those that were training, regardless.

\section{Social}

Intuitively, a training in martial arts that is based on a learning from a teacher will almost inevitability include a social dimension to the things that are 
noted in survey responses as well as interviews. Whilst the data collection tools focused primarily on the perceived outcomes to learning a martial art in terms of health and well-being - how, and in what ways, these benefits - if any - were resultant were left open. This meant that participants in both the survey and the interviews could make reference to the factors they considered important, as opposed to those that were predetermined, thus making their responses much more authentic. In relation to questions on well-being and anxiety, a network of support, friendships, community belonging and social happiness were key for many of those that replied. From Table 5.2, we can see that 'meeting new people' was important to learning a martial art. Examples of some of the reasons are given below:

There's a sense in the Dojo we come together, we practice together, the calmness sets in so I think it's a double benefit,

Beyond the immediate personal benefits, practising aikido has allowed me to access a supportive network of people who have a similar philosophy to life

(survey response)

Martial arts bring focus and an outlet for when other areas of life are not going well. In addition, my experience if that the dojo is like a family people are always happy to see you and accept you at face value, and value what you bring to their training

(survey response)

The friendships that I've made through martial arts are often stronger than those through made through other things. I feel like I have a good support network through my martial arts friends

Both the survey and interviewee responses made numerous comments on the importance of belonging to a group and the sense of identity that came from this. Indeed, as seen in Table 5.2, 'feeling like part of a club/community' was important for 118 of those who responded (71\%). For many, individual and lifelong friendships were formed through martial arts training and were, for some, based on a deep bond of trust that also represented a form of additional family, a martial arts family that could be relied and depended on. From Table 5.2, we can see that this was also important for $44 \%(n=71)$ of those that responded to this question and is clearly illustrated in the longer interview excerpt below:

I think you know, that just kind of on one level...I mean there's actually kind of research that shows that doing dance-like more...more synchronized movement together is actually hugely beneficial for people, you 
know, they do, they have a sense of community, they have a sense that, you know, they get out of isolation...I mean, you know, the fact of the matter is, you know, human beings, you know, we think that we are alone in the world, but we are not we're just a reflection of everything and that because none of us is knitted together though relationships so, you know, the more we can get, you know, in these types of synchronized...relationships that focus not, just on our own, small problems, you know, that we understand kind of immediately that we are a part of the bigger picture. I think that, you know, that that does help, we come together and we take a class for an hour, or three a week, and then we go out into the rest of our community and our...you know I do think that having a more... having kind of a dojo where the focused intention is this pliancy...whether it's physical or mental, if we really look at that and if we really take that into our heart, then whether we are skilled at the actual form or not, is secondary to whether we start reflecting on that, whether we're driving down the freeway and we let somebody pass us or listen to our spouse or our friends.

For others, friendships outside of the class were not important or at least not as important as the regularity of meeting up with like-minded people, who shared similar interest and who took a general interest in your life and general wellbeing. This sense of community belonging was very apparent and in a number of ways. Whether because of the type of martial arts being practised, the teacher's class attended or being a body of students training together, the idea which is inferred is that coming together created a social bond that promoted a specific, yet almost transcendental, type of well-being and social happiness:

...if you lose your mobility and the flexibility, you're going to start to become isolated and cut off from society, the community around you... so it's very good.

$$
25-65+\text { years }
$$

I've made...some very good friends...I've got very good friends who do it, you know. Er... um...it's nice, it's like going to church and just meeting friends there, you know, it's like that, it really is, you know. Yeah, and everyone helps each other, if you're struggling you help them, you know, and also they'll help you, you know. Um...so it's good, it's a lovely experience

Examples from the survey are given below:

- I like being part of a community of like-minded people.

- There is a real community and common interest among true martial artists; I've been lucky to have met some great people. 
- There is this sense of community, of coming together and doing the same thing.

- The community benefits too; there are community cultural benefits.

- Training in a class with a structure and goals is important because it pushes you outside your comfort zone.

The aforementioned comments make note of the pleasure that comes from being with a group of people following the same movements. For others, it was the structure to the week - important for $30 \%(n=48)$, the connecting with others $(30 \%, n=39 \%)$ and the sense of shared purpose that contributed to this sense of social good. 'Feeling a useful part of things,' for example, was important for $58 \%(n=170)$ of those in the survey. Whilst the social dimensions of martial arts were myriad, what is clear that learning martial arts had a significant and important social dimension that was seen as a key contributory factor to the broader health and mental well-being outcomes that were noted by the participants in the research. This can clearly be seen below:

So there is a nice sense of being an individual in a group that has a single purpose. And I guess it's a little bit, I'm in a singing group as well, I guess it's a little bit like that. That you are very different people but you have this one thing that's very strongly in common. And when we do do partner work you, we make an effort to partner with different people all the time so you're with different, sometimes I'm working with very large young man, sometimes with another older woman and it's great to feel the connection with the physical connection as well as the mental connection with people who are so different from each other.

I can socialise, but it's not why I do it, the, when everybody has a cup of tea together at the end of the class, in the council art centre where we practice in the dance studio, and I'm going off to do shopping or something, I'm aware that it, I don't do it for social reasons. A lot of people do, if you're talking about connecting communities.

\section{Management of stress and the link to health and well-being}

Overwhelmingly, mental well-being, via the management of stress, was seen as one of the key contributory sources of support for health through the practice of martial arts. In addition, a greater sense of physical self-confidence and a sense of efficacy and control over well-being were also highly important, in terms of the consequences of participating in their art. These ranged from the perceived benefits of connecting with the self, providing focus, as 
well as managing anxiety. Participants in the survey were asked to indicate whether martial arts were beneficial to a number of specific areas connected to well-being and were taken from standardised measures. Responses to these questions ranged from 'no more than usual,' a 'bit more than usual' or 'much more than usual.' From those that responded, martial arts were considered as contributing directly to 'enjoyment of day to day activities' $(79 \%, n=231)$, 'feeling happier' (79\%, $n=231)$ and 'ability to make decisions' $(57 \%, n=165)$.

Many of the open-ended comments related to the ability to be physically and mentally 'in the present' as a meaningful way to manage anxiety. The examples that exemplify these areas are also numerous and wide ranging, and just a small selection is included here:

Well I've never, I've never been a very embodied person and I had a difficult childhood. But I've found that qigong and tai chi are just, [and I'm a psychotherapist], are integrating me and bringing me into my body, and integrating my psyche and my psychology with my body...everything in a really, really good way...it's very good for depression and it's good for the psyche, it's good for the mind, for the brain. It's good for the brain because you're using alternate bits of the brain and moving in different directions.

I think also it's quite calming. I have high blood pressure, but I've found that my blood pressure has stabilised quite a lot. And I'm sort of concentrating on the way I move. And also thinking how I move. And not getting stressed, in a sense. Sort of, almost like inward. Yeah. I mean, I dread to say the word, mindfulness, because that is it...I mean, we do exercises at the beginning which we concentrate on our breathing, and yes, OK, we have thoughts that come into our heads, but it does make you focus and think about how your body moves, and what you're doing. I'm a busy person, I don't like sitting down. And my children have always said to me, mum, you never sit down. ... But I do find it useful, because it means I have to concentrate for that length of time, quietly. And I think everybody is, I hate to use the word 'stress' because that's a bit overused, but everybody has concerns these days, and I think that it does help you. I said it helps you with balance, because I really do think it does help with balance. And I said it's calmed me down, in the sense that I feel more comfortable in myself.

I don't know, it seems to get into you and you want to be good at it and it makes you feel good as well and especially as a young lad on the streets of South London, you felt far better than you would have done, had you not learnt anything, but as a young lad it was great for the confidence, but it was also addictive because you want to get that next belt, or you want to be better than that other guy, or you've sparred with someone and they made you feel stupid. So, you think, right, well, I'm going to really try hard and this time next year I'll be better than that. It's part of what 
you are essentially, what defines you isn't it? [It's a] feeling of well-being I guess. A feeling of you're making progress to a target.

You come out feeling, well in most cases you feel much better than when you've started. It is, the practice, my own personal practice is hugely important because it roots me into the here and now in a rather complex life that I lead and these periods of calm

I wouldn't be without it. So, I would recommend it, but it's not as simple in terms of promoting health, [it's] all aspects of health, from mental, emotional, physical, it isn't straightforward.

The mental well-being benefits are complex and interconnected, in nuanced ways, with both the practice itself and the social dimensions that interact with these - both within and outside of the dojo. The following excerpt beautifully highlights the challenge of trying to capture some of this nuance:

...the calmness sets in, so I think it's a double benefit. People who practise Qigong and Tai Chi I think are paying more attention. We're more mindful. Anyway, I think that because I'm more centred and calmer, and I'm able to listen to others more, more able...to be there for my friends, they're able to be there for me, and I just think again, because I can be mindful and attentive that's certainly enhanced my relationships. Over the years have found that I'm better at being aware of myself, how much anxiety I have over something and [now] doing something about that. And [my practice] has really helped with that. Well, it's like a continuous learning...I enjoy that I can see my body slowly but surely, or actually not seeing it... [but] maybe I am actually making changes and improvement. So that continuous learning aspect I really love

I feel a greater harmony with the natural world. I have been known to be doing Qi Gong in the garden and a mole has popped up!

From the survey and its open-ended survey responses, it was clear that martial arts offered a release from stress for the very many of those who responded. It was seen as a source of support 'when feeling under strain' (67\%, $n=194)$. Whether this was through the simple process of disconnecting from the outside world, which is afforded by a weekly class, or through a greater awareness of the physical self and, by association, the physical tensions, $77 \%$ $(n=225)$ felt an increased 'sense of overcoming difficulties.' For others, martial arts offered the option to adopt techniques, such as particular stances, 
breathing techniques or mental focus that could be used in a variety of spaces, including home and the workplace. A sense of 'calmness' and an ability to 'deal with stress' were also noted by many, whilst 223 (76\%) felt that it helped with sleeping. In the open-ended comments on the survey, numerous references were made to 'happiness' as something associated with martial arts practice. Some examples of these are given below:

- When I am participating in martial arts, my happiness grows.

- It has generally improved happiness due to mental discipline and being able to relax. Letting worries drift away.

- Practice provides a refuge from events that would otherwise greatly impact my overall happiness.

- Happiness, in my book, goes along with mind and body confidence and that is something I am aware of since starting martial arts and feel more in control.

Physical confidence also links very explicitly with mental well-being. Numerous examples that highlight experiences of bullying as well as how these experiences led to low self-esteem were offered by some, as well as how weight and physical statute were also connected to ideas that we infer to illustrate lower levels of self-confidence. For example, one respondent discussed how as a shorter than average woman, she lacked confidence and felt vulnerable but learning karate had made her feel 'tall on the inside':

I am a small girl. Martial arts makes me feel less small, less vulnerable and gives me more self-confidence in everyday situations, not just when I am walking alone at night but also at work/studies as I know that even if I am small, I can achieve big things! (from survey)

Physical confidence was also important in just a couple of examples where an experience of violence had led to the initial motivation to learn a system of 'self-defence.' For these, training resulted not just in increased physical confidence but also in a more general sense of confidence and feelings of empowerment:

Participating in martial arts has increased my confidence which has allowed me to believe in myself allowing me to overcome situations that would be overwhelming before

(from survey)

...confidence that helps me make decisions rather than delaying them

Controlling and managing emotions were also noted by some:

It has given me discipline and good control on my emotions

(from survey) 
Other illustrative examples, from the survey, are given below:

- It makes me feel settled.

- I feel calm and it rolls over into my everyday life.

- It gives me a channel for my anger.

- If I get angry or perturbed, I gain control more quickly and regain control over my mental [self] - this helps with my overall well-being.

- Improved my attitude with people.

- Taught me to let go of my emotions.

- I have learned to be patient.

The following, much longer extract from an interview, highlights very clearly the multiple ways that karate gave support to the emotional, physical and mental health needs of one young man:

I was bullied [and so] I had some aggression issues, so my teacher and my parents...decided that martial arts wasn't for me, they feared that it would make me more dangerous since I had these aggression issues. Because what happened was that I would really, always answer in the only way that I knew how which was violence. And um, after a while, I um, I finished first years at school and I changed schools and er, one of my teachers, actually my maths and science teacher, he was er, he was a trainer in karate and we were discussing natural reaction patterns and behaviours in the sciences and he started talking about karate training and how that had changed a lot of his typical reactions patterns and er ... so I went up to him after class and I spoke to him and he said actually we have a beginners class starting today so if you want to join me please do. So I literally sloped off, without telling my mother, when I was thirteen and joined the beginners class. And I'd been about four times, six times and then I told my mum and she...she took it pretty well she said, yeah well you know, the bullying was years ago now so this is probably fine, but, behave. And I've never looked back.... I mean I could have been stabbed, I could have been stabbed so karate saved my life, in one way or another, because I was bullied to the extent of selfharm, and... and I attempted suicide, and er I think karate, the focus and the companionship and friendships laid bare, really, really helped me ... what happened with me was that I was pretty low on self-esteem when I started, and I still occasionally go back into that inner lad twenty plus years later, I still go back into that mindset of er, of um, not feeling adequate, and um, it's interesting because karate gave me at least the tools to cope for a while and the tools to focus, and er after a few years of training - six, seven, eight years - um I started gaining self-confidence when I realized from feedback on this that I was actually quite skilled at what I did. But that took some time...um, I remember meeting, when I was maybe twenty, and had trained for seven or eight years, I was 
out with some friends, a night on the town and um, I didn't drink any alcohol at the time um, and I remember being confronted by a number of my old bullies from school... and um, they basically picked up where they left off, starting with...wanting to pick a fight and er, and er...I've always been a chubby kid so they picked up on that again, and then one of er, one of my friends said kind of loudly, you know, you should back down because he knows karate and they, just seeing him saying that, they backed away...strangely enough, [I felt] empowered, because this is why they didn't do anything... and then I said, to my friend later that these guys, there were three guys, I couldn't have taken these three guys on, whether I wanted to or not... [but] they believed you could and er, my friend said, well, you know, I've seen you and you are really good at what you do now, and there was some...some sort of er, well um, what's the word...there was a mismatch between my image of myself and others...other peoples' image of myself...[now] I would say that, um, I've become a lot more patient...Um, and I would say that er, my confidence, and er my self-confidence and my image in myself, that has improved tenfold.

Many of those in the survey made note of life changing events, such as bereavement, redundancy, marriage break ups as examples of the ways that martial arts help with coping with these. It is important to note that martial arts themselves were not the fix, but more the structure, routine and the relationship made that provided a mental 'safety-net' which was an influential motivator:

Over the years I have had a couple of spells in hospital and what kept me going was [gosh] I've got to get out of here and get back to training. I can't lie in a hospital bed...I've got to get well and get back to it....I don't think it's the karate that made me recover, it was the thought of not being able to do it, that drove me to get off my arse and get better.

Perhaps one the most striking things that appears to be apparent within the data is the ways that participating in martial arts was considered as offering a modicum of self-efficacy and ownership to health. Numerous references were made to this area. Below are just a few examples of these:

- [it] has given me the ability to maintain a good level of aerobic health and control over asthma and anxiety due to calmness of mind.

- [a]deeper involvement in tai chi enabled me to come off of anxiety medication brought on by stress and personal grief. I am far more able to control anxiety due to relaxation bought about by daily training in tai chi.

- Control over stress. 
- If I become angry or perturbed, I can quickly regain control of my mental [self] and relax and make better decisions. Being in control of one's mental [self] helps with overall well-being.

- It has given me discipline and control over my emotions

- Well-being increases with greater control of balance and co-ordination.

- I have taken ownership - I am more aware of my decline in terms of balance and flexibility which means I have time to work on this now. Being more aware of breathing, posture and energy and how all things interconnect to impact in general well-being both physically and in relation to mentally.

- I feel empowered by feeling that I am taking ownership of my health.

Indeed, from the survey, $84 \%$ of those that responded $(n=242)$ said that their martial arts practice helps deal with 'worries over health and well-being' when older. As noted earlier in this book, mental well-being is a growing concern. However, martial arts for many of those included here were considered as providing a meaningful mechanism by which to manage some of the stress that was experienced. The interview quote below illustrates this well:

...I'm actually a very anxious person OK? Because I tend to over analyse things. My brain is always whizzing away and it's constantly playing through scenarios to work out what's going to happen and so I'm ready for things if they happen. Because a lot of things that could happen but almost certainly won't happen are bad things so, if I'm playing through those and thinking what would have happened blah, blah, blah, blah, blah. Now, one of the things that tai chi has helped a lot with and this is very curious actually, I didn't expect this at all. Is it's made me understand, this is really weird to say this perhaps, it's made me understand that I have control over nothing except to some extent myself. And if I really, if all I can do is have some control over myself in this world of potentially terrifying things, then I can exercise that control and that gives me some kind of grip of the universe. And that's what, I mean tai chi just like I said, the routine to relax and things but even just doing the tai chi itself. This morning I went into the garden and did half an hour's tai chi and I've tried doing tai chi in the garden in the morning. I get up, just put on my tracky [sic], training trousers and a top or something, a fleecy top or something to keep warm and there's a hat you know. And I go and do tai chi in the garden and I stand or do a bit of warm up, chi gong stuff and then do standing or silk reeling or both for something like half an hour or three quarters of an hour in the morning. Just doing that in the garden, it can somewhat, kind of control over me. And it's just actually as you said, your own mental attitude to things changes the world for you so this realisation that I only have control over me and other people's expectations and things are their problem rather than mine. 
It stands to reason that these benefits will vary of course, and the impact experienced will also vary depending on how often an individual attends to their training as well as how long they have been learning for. This is confirmed with Analysis of Variance (ANOVA) tests of variance.

\section{Chapter summary}

In this chapter, some of the ways that martial arts are considered as impacting on well-being were considered. It is important to reiterate once again that the data presented highlight the connections between practice and outcomes from the perspective of those included and not in terms of causality. This is important to make clear, as whilst we do not claim any causal connection between martial arts practice and well-being in an empirical sense, what we are doing is shining a light on the relationships and connections that those included in this research make for themselves. Clearly, 'well-being' is complex and spans intellectual, social and physical domains, to name but a few. Underpinning all of these is the idea that intellectual stimulation, social connections and physical 'confidence' are highly relevant dimensions to well-being, albeit in varying degrees of importance, and to broader ideas of health. The data in this chapter have also highlighted the significance of 'ownership' of health and the interconnection between health, self-efficacy and well-being in this area. A sense of 'taking charge' is important in feeling that health and well-being can be controlled, rather than 'controlling.' For many, martial arts offered a vehicle to learn about one's own body and to see how connected the physical and mental selves are. For example, the connection between stresses as evident in the physical was a key means by which managing stress was possible. By recognising the connection between mind and body, it then becomes easier to find ways to manage both. Such awareness is crucial to maintaining and managing mental health. 


\section{Connecting communities and promoting health}

\section{Introduction}

This book set out to explore empirically how and in what way martial arts could be considered as contributing to health and well-being from the perspective of those that practised them. Whilst we make no claims as to the generalisability of our findings, the replicability or indeed, the uniqueness of our findings to martial arts per se, what we have done is to explore, to some degree, what it means 'to do' martial arts from the point of view of those that practise them. The significance and importance of this type of focus are twofold: firstly because if, as discussed in Chapter 2, our behaviours are shaped by our beliefs, then it is important to explore those beliefs. Secondly, if our behaviours then reinforce those beliefs, then it seems much more relevant to consider individual perspectives and beliefs concerning their health and well-being than attempt to isolate and test, in a more controlled, experimental way, the impact of these.

As highlighted earlier in Chapter 1, health and well-being are of phenomenal importance in the 21st century. With an ageing population and increasingly sedentary lifestyles, coupled with a growth in poor diet that link directly to increasing rates of obesity, diabetes and cardiovascular deaths, a focus on health and lifestyle is clearly an important one. Together with these concerns, there is also a growing crisis within the field of mental health and well-being, one that has caught the attention of a range of professionals and media, as well as the general public more widely. Increasing rates of stress and depression - as well as a growing concern in relation to the mental wellbeing of societies young people - have shone a spotlight on this priority area. In short, we are facing a global pandemic in terms of health and well-being. Alongside this, there is also a growing recognition that change is needed. The rise of vegetarianism/veganism, interest in particular diets, such as wheat free and sugar free, as well as the rise in things such as acupuncture, personalised nutritionists, diet apps and personal trainers, clearly highlights a growing degree of awareness. With global health issues and associated medical costs predicted to grow exponentially, a focus on behaviours and the perception 
of those behaviours is, therefore, both timely and significant. In this chapter, we draw together the findings presented in Chapters 3-5 and consider the significance of these in relation to the issues raised in the earlier chapters. Specifically, in this chapter, we first begin by recapping, very briefly, on the key theoretical ideas used to frame this research before linking these ideas to the data presented. We also discuss the broader relevance of research of this kind. Specifically, we highlight how our theoretical understanding of the role of the individual as well as society more broadly has been extended by combining different theoretical lenses, in this case, psychological, sociological and philosophical, to the significance of understanding behaviour and beliefs as well as the broader field of health and well-being.

As discussed in Chapter 2, differing academic disciplines explore the area of 'behaviour' with differing emphasis depending on the field. The key ideas presented earlier in Chapter 2 can very loosely be organised under five themes which we briefly represent below:

1 Behaviourism - Largely focused on the way that behaviours and actions can be understood as a consequence of either rewards or punishment, where the behaviours and actions that we want are reinforced whilst those we do not are punished. Whilst it may no longer be particularly theoretically influential due to a lack of a consideration of the role of environment or other external stimuli, the notion of learned behaviour does make intuitive sense to some degree; the desire to do the things we find pleasing whilst avoiding those that cause pain or discomfort resonates. There is, however, an inherent assumption that one is able to rationalise and think of behaviour in terms of cause and effect, and on the basis of understanding.

2 Social cognitive theory - In this model, the environment becomes a key mediator and is very much focused on the way behaviour is learned via observation. Thus, behaviour is very much a social process. Key to the learning via observations is the notion of imitation - where we mirror the behaviours of those we admire and want to be like. Imitation is useful in explaining the repetition of both bad and good practices across families and different groups. However more than just observing, key to understanding behaviour and practices is the role of motivation - motivation to imitate. It is important to be clear though that motivation is not simply a desire to do something, or not, but is strongly connected to ideas of self-efficacy - how strongly one believes in one's ability to have control over one's life. Motivation, then, requires a sense of self-efficacy that is developed through (a) mastery experiences, (b) social models, (c) social persuasion and (d) stress reactors. Behaviour, then, was understood as a process of self-efficacy, motivation, reward and punishment (which can be internal, external, positive or negative). 
3 Society - For sociologists, human beings are understood to be dependent on each other for their survival, being unable to exist in isolation of each other when resources are scarce. The social groups that emerge by this coming together are important as they determine and reinforce the practices that are considered important, whilst penalising those that are not. Behaviour and practices are, thus, a reflection of the values and norms of different groups, meaning that behaviour is not static or fixed but varies across time, space and place. They are also key as they promote a sense of belonging and social identity. However, sociologists are also clear that behaviours and preferences for different practices vary even within the same society, largely because they are highly dependent on access to resources, which are not equally distributed.

4 Culture - Groups that practise similar behaviours are essential in bringing people together, reinforcing bonds and promoting an important sense of self. Social practices that involve a collective gathering mediate behaviour because of the associated rules required for group membership, as and this has been evident across the millennia. Ritualistic practices, from tribal drumming, military marching, football chanting and nightclub dancing, for example, create a sense of solidarity and belonging which, in turn, promote the feelings of well-being.

5 Eastern philosophy - Eastern philosophies very much underpin the myriad martial arts that are practised currently and could be considered as tying very explicitly to the behaviours and practices associated with those arts. Strongly influenced by Taoism, Confucianism and Buddhism, notions of wellness and self-mastery are key. Alongside their importance to martial arts, Eastern philosophy also offers an important framework for understanding the topics that this book is concerned with. As noted earlier in Chapter 2, Zhu Xi stated that all action must first be preceded by a thought or intention. Thus, it is important to understand those intentions through personalised reflections of self-learning so that we can then draw larger and broader conclusions.

The ideas presented above provide a range of differing, yet somewhat complimentary ideas to help explain behaviour. These can offer useful windows for understanding varying lifestyle practices that may, or may not be, conducive to health and well-being. In the rest of this chapter, we consider these ideas in relation to the data presented.

\section{Motivations to learn}

The data presented for both the martial arts teachers and the student practitioners - and across the age ranges - concerning the impetus and motivation to take up a martial art were myriad and varied; however, there were 
some general themes that were evident. Motivations could largely be seen as organised around five key areas: (1) physical - to do something active and/ or to become fitter; (2) health - a desire to support heath, either proactively or reactively; (3) a desire to do something that required learning; (4) more generalised, such as self-defence, self-confidence, role models or chance and (5) mental well-being. Each of these will be briefly discussed.

Physical - Motivations for undertaking a martial art that were based on physical reasons tended to cluster around the desire for support with things such as flexibility, agility and mobility. A desire for more generalised fitness was also key, alongside a feeling that the gym was either not suited to their needs or was not able to satisfy what it was they felt they needed. As noted in Chapter 4, a desire for a sense of physical well-being was also important. This is interesting as whilst some of the respondents noted a general sense of 'needing something more,' they were not necessarily able to articulate exactly what this 'something' is. Yet, recognising that this 'something more' was connected to the physical self was what provided an impetus to find an avenue to address it. Physical motivations were also very often associated with notions of physical maintenance and prevention of physical decline, in that activities were sought to either improve or maintain current levels of physicality or, at the very least, prevent further decline.

Health - As might be expected, health was a big motivator for many of those included in the research, with support for 'general' health or specific health conditions forming a strong motivation for taking up a martial art. Specific conditions included things such as arthritis, asthma, cancer and other life changing illnesses as well as things such as chronic back pain. Health motivations were particularly true for many of the older people, who saw taking up a martial art as a means of maintaining health as well as taking ownership of it. This sense of proactivity, whilst often initially emerging from a specific health need, is also interesting as it highlights an aspect of self-reflection and the recognition that there is a personal responsibility for one's own health. Many of those included in this research had a very generalised concern with health and fitness which provided a source of a motivation; yet, there was also clear evidence that initial motivations were a means of remedial or preventative support for the areas they were concerned about.

Learning - Perhaps unsurprisingly, a desire for self-improvement through learning, as well as a yearning to learn something with perceived depth and layers, was an important influencer in motivating a much small number of respondents in this research to take up a martial art. Again, a sense of searching for 'something' to address an unspecified need was noted. Wanting something that involved learning was because it was seen as a means to keep the brain active, provided avenues for growth and offered access to learning about new things. Indeed, these were important rationales for learning a martial art, which was considered to offer opportunities for learning that were perceived as not afforded by simply going to the gym. 
General - More generalised reasons for taking up a martial art were sometimes connected to feelings of not fitting in or not being good enough at other types of sports. For example, some of the respondents noted team sports as something they found challenging or struggled with. Team sports can exaggerate feelings of social awkwardness and can tend to emphasise feelings of isolation in those that are less confident, either socially or physically. Such ideas are often rooted in childhood experiences at school. Other more generalised motivations, and perhaps rather unsurprisingly, were the role models. Just as Bandura noted, role models offer an idea of someone we would like to be - in this instance from the martial art world of movies, names that are synonymous with this genre such as Bruce Lee and David Carradine. These examples clearly emphasise the social dimension of learning. Finally, selfdefence was also noted as a key motivator for many of the respondents in this research, whether because a desire for self-defence was a response to an event that had already happened, a generalised feeling of vulnerability, sometimes due to physicality, or simply to create a sense of confidence in one's own ability to protect oneself. Motivations in these examples can be considered as reflective, in that they are framed within a clear sense of 'self' and identity.

Mental well-being - Whilst the mention of finding ways to manage and deal with stress as a reason for taking a martial art is perhaps not surprising, what was striking in the data was the number of times that stress was mentioned. Alongside stress, a desire to improve self-confidence was also noted. A clear connection to self-confidence, stress and mental health was significant. Interestingly for those that did note their mental well-being, a clear connection between the mental and physical self was evident, as was the recognition that the physical self was clearly connected to other aspects of self. How much of this awareness was more the benefit of hindsight is not clear. However, interesting in its own right is that it was noted as a motivating factor.

It is clear that a range of factors are influential in motivating those included in this research to take up a martial art. Whether to manage a particular health concern, support general fitness and physical well-being or support a more generalised desire for mental well-being, there is evidence that notions of self-efficacy offer some understanding as the mechanism that underpins these motivations. If, as Bandura (1998) states, self-efficacy is concerned with setting goals, which are motivated by a belief in an ability to produce results through one's efforts and endeavours, then clearly, identifying a need and then acting on that need - in this case by taking up a martial art - is very evident here. In the case of initial motivations, at least two of the influencers that Bandura notes in Chapter 2 as important types of self-efficacy are distinct in the data, these being social models and stress reactors. In this research, social models being those from the movies, whilst stress reactors being the ability to assess physical and emotional states and then act to alter how these are experienced. As Bandura (1997) and Schwarzer and Fuchs (1997) would undoubtedly agree, participants in this research demonstrated high levels of 
self-efficacy, with the data in this research offering a great illustrative example of attempting mastery over stressors. Alongside this, we see a clear example of regulating behaviours in ways that attempt to deal with health and wellbeing in a positive and meaningful way.

\section{Perceived benefits of martial arts}

This research was concerned with the perceptions of those who took part, as to the benefits they associated with their martial arts practice. It is important to remind the reader that this is a study of the observations and beliefs of those that took part and so is not intended to 'prove' a link between martial art and the outcomes respondents noted. However, that said, it is fair to say that participants were overwhelmingly positive in terms of the benefits that they experienced, from even a small degree of participation in a martial art. Benefits were direct, in terms of improvements in health, fitness and mental well-being but also more tangential too, in relation to the behaviours and practices that supported these areas. In this section, we discuss these benefits briefly, focusing first on health and then on well-being.

\section{Health benefits}

Improvements to health were one of the key benefits associated with martial arts, perhaps unsurprisingly given that it is a form of practice requiring physical exertion. It is also interesting to see just how many of those who responded felt that there had been a general improvement in their health, not just their health, but also their awareness and understanding of their health. Specific improvements were noted in general things such as posture, balance and flexibility. Given that martial arts focus on specific movements, as well as postures and stances that require an ability to understand, sense and control the physical self, it is heartening that the data show how these skills develop, not only through practise but also because of the greater physical understanding and awareness noted by many of the participants in this research. A greater sense of connection to their body was evident in awareness and behaviours linked to health, in areas such as diet, caffeine and alcohol consumption, as well as sugar to name but a few. It could be that along with the development of skills in martial art, a much greater attunement to the body and understanding of its needs was a consequence. Certainly, an improved awareness in these areas was noted by many.

Support for specific health conditions was also a perceived and specific key health benefit, particularly many of the health conditions associated with lifestyle, for example, conditions such as weight, diabetes and blood pressure, as noted in Chapters 1 and 2. Other illnesses and chronic conditions were also considered to have improved significantly, including things such as asthma, mobility issues and conditions such as arthritis. Martial arts also inspired an 
increased confidence amongst those facing more challenging diseases such as Parkinson's and cancer, allowing those battling these particular conditions an opportunity to feel that they were 'doing something' to support their health and well-being. This notion of proactivity was important in promoting a sense of 'feeling good,' partly because such illnesses can create a sense of helplessness, on occasion. A feeling of powerlessness can often feel alleviated by activities such as martial art. Perhaps even more importantly though was the perceived sense of confidence that perceived benefits were directly connected to their practice - in this case $80 \%$ believed that the improvements in health and health awareness were directly connected to their martial arts practice, with $90 \%$ of martial arts teachers saying the same.

The health benefits perceived by participants are heartening. As Nettleton (2013) notes, the latter half of the 20th century was marked by a shift towards illness prevention instead of cure in health policy documents, primarily for the reasons noted in earlier chapters. Things such as an ageing population, a movement from infectious diseases to those much more connected to lifestyle, as well as the overwhelming financial burdens on public spending. In this research, efficacy - a key theme that very much underpinned the changing public health discourse Nettleton discussed - was clearly evident. However, the data also suggest that the health and fitness impetus underpinning the desire to 'do something' was very much the result of an individualistic approach to health, as opposed to a response to larger public health narratives. Of course, not all participants attributed the health benefits they noted to their martial arts practice alone. Instead, a range of factors were also important, yet still factors that were mediated by and through their practice.

\section{Health awareness}

Arguably, a key determinant to changes in the practice that supports health is exposure to the knowledge and information that facilitates a shift in awareness. However, Graham (1984) suggests that being informed is not the pre-requisite to changing the practice that supports health. He argued that with knowing what one should do only resulted in healthy compromises. Instead, Thorogood (2002) argues that shifting the health beliefs and awareness of non-professionals is the best way to deal with the complexity and challenges of health on a structural level. In this research, health awareness was explored in relation to how martial arts practice had promoted a much greater understanding. As we saw earlier, awareness was a key finding.

As noted in Chapter 4, we were not concerned with how or why greater health awareness develops in the casual sense, only whether the belief of participants was that it did improve. For the non-teachers of martial arts in this research, three quarters believed that they had much more awareness of their general health since starting their martial arts, with the same number also believing that the actual health had actually improved. When explored 
further, $80 \%$ expressed confidence that the improvements and awareness that they believed they experienced were directly related to their martial arts practice. An array of specific health conditions was mentioned, in terms of how martial arts practice supported these areas, conditions such as Parkinson's, asthma, sciatica and arthritis. Support for conditions such as cancer and heart disease was also noted. Arguably, the rationale for understanding this improvement could be intuitively made as a result of exercise and movements that support the pain and management of different health issues. However, improvement in awareness of factors associated with health and physical well-being is a different issue. What is it about martial arts practice that promotes greater physical and health understanding and awareness? Partly, some of this could be understood as resulting from exposure to information and knowledge from an experienced and knowledgeable practitioner, the martial arts teacher. However, being in a class with other students, who may also have their own issues, provides a valuable opportunity to share and talk about experiences. The social dimension to learning is, therefore, also highly relevant. An understanding of where the difficulties are in your body and the things that you begin to be able to do through your practice means that learning is experiential. Experiencing improvement suggests a receptivity to extending understanding as to the way that what we do and its impacts on our everyday life and health more generally. As Zhu Xi notes, we must first experience before we can understand. Martial arts and its practice offer a perfect window into these understandings. In a theoretical sense, the somewhat outdated theories of Skinner and behaviourism would seem to apply here, that is, learning as a process of cause and effect. However, social models of learning are perhaps much more relevant. This is because learning about health and the benefits of practice suggests that notions of learning through observing and imitating others are also important, it is not just a process of action and response. Bandura's (1998) ideas of health self-efficacy seem to be highly appropriate for understanding improvements in health and health awareness. As he notes, self-efficacy does not operate in isolation but is mediated by the environment. Thus, seeing people similar to yourself, in terms of age, shape and health concerns, can be highly motivating and encouraging. It facilitates an awareness of the 'stress reactors' that Bandura also notes as important. For Bandura, changing habits is what is key to promoting greater health and health awareness. These ideas seem highly plausible in offering an explanation to the broader areas of awareness and changes in practice noted by some participants, such as improvements in awareness around sleep, sugar, caffeine and other aspects that contribute to unhealthy lifestyles. It is clear though that these benefits are scaffolded in the traditional sense, in that the greater the frequency of practice, the greater the health awareness and perceived benefits. It could be argued then that health and health awareness can be addressed by offering opportunities to learn through observation and through experience. Through repetition and practice, a sense of self 'mastery' 
(Bandura, 1997) is possible, providing a greater understanding of the connection between what one does and how one feels. In some respects, practice and beliefs can create a sort of self-fulfilling prophecy. To illustrate, if I believe something is good for me, I will more likely feel good about my practice and/ or be motivated to practise more. The more I practise, the more likely I am to feel that my health and well-being have improved.

Greater health awareness can also promote a sense of empowerment that, in turn, offers an avenue to mediate worries and concerns regarding health, both for now and in the future. In this research, 'ownership' of health appears as a fundamentally key contributing factor behind those that felt they experienced improved health and health awareness. Having a sense of ownership and control over one's own well-being is important to feeling proactive in managing health in the present and the future. This suggests that opportunities to learn through activities such as martial arts should not be left to chance. Clearly, if health and physical well-being are best supported by dealing with it proactively, then in future planning, it makes sense to have a move towards a less traditional school sports curriculum. Instead, a curriculum that offers martial arts, alongside football, rugby, hockey and basketball, would enable young people to embed the learning of health and health management at a very early age. This type of learning sets a strong foundation for later life. In addition, classes for other vulnerable groups would also help in dealing with the current health crisis. Currently though, martial arts are not centrally funded. Providing access to these activities though, through national health services, health insurance and educational budgets, would ensure fair and equitable access to the types of practices that support not just the individual but broader society too.

\section{Well-being}

As noted in the introduction to this book, the issue of mental health and general mental well-being is a rapidly increasing area, and of huge importance. The issue of mental ill health is a global phenomenon, and with consequences for individuals and whole societies. Alongside mental ill health is the issue of mental well-being. As discussed in Chapter 1, mental well-being is an area of concern across both ends of the age continuum, that is, from the young and the very old. Stress, anxiety and loneliness are just some of the manifestations of poor mental well-being, which also links to an ability to manage everyday life.

It is important in any book that is looking at health and health awareness to consider well-being. This is because well-being links and interconnects so directly with general physical health. Well-being impacts on how we feel about our lives and will inevitably impact on levels of self-efficacy in relation to health and mental well-being. In this next section, we discuss briefly the findings on well-being as related to confidence, learning and the social domains of well-being, as well as stress more generally. As a reminder, mental 
well-being in this book is that as is defined by MIND, a British mental health charity. It, therefore, relates to subjective opinions on self-confidence, selfesteem and sense of an ability to cope with everyday stresses. Mental ill health was not a feature of this research and where it is mentioned, it is as an emergent theme from within the data. As a useful measure to conceptualise and operationalise well-being, we collected data using British government measures on well-being and anxiety. And again, data connected to this area were based on subjective perceptions.

Interestingly, in this research, participants overwhelmingly believed that since beginning their martial arts practice, they felt happier, more satisfied with life as well as less anxious. The open-ended comments from within the survey suggested that these responses could be understood as a response to a range of factors. Self-confidence is instinctively an important aspect of mental well-being. A generalised sense of self-confidence, or specifically increased confidence that emerged through the development of the physical self, could clearly be seen as linking with Bandura's (1998) ideas of selfefficacy once more. With self-discipline comes greater resilience and focus which, in turn, interacts to promote a sense of self mastery. Once again, and very strongly noted in the data, was the notion of 'ownership' - ownership of mental well-being promoting confidence in this case. Feeling that one can have some control over how one feels is profoundly powerful for many. And, as we also noted, even though not everyone recorded such positive outcomes for self-confidence, even for those who recorded much lower levels of mental well-being, this was largely attributed to a greater understanding of what needed to change in the person's life to feel better and have a greater feeling of well-being. So conversely, martial arts were still seen as valuable in this area although indirectly. As Schwarzer and Fuchs (1997) note, when confidence is low and levels of self-efficacy are also low, people tend to engage in health-damaging activities, such as smoking and drinking. What martial arts could be seen as doing then, at least in this instance, is encouraging. Selfmastery the idea that through perseverance and effort life begins to feel more manageable, promoting consequences that can be felt through many aspects of everyday life. Arguably, in this instance, martial arts practice is an instigator to other activities that may also lead to and promote mental well-being, such as weight loss and time out for relaxation.

There are other consequences to engaging in an activity such as martial arts that is so much more than just a physical practice for many. At least for those that choose to make it so, a way of life with deep philosophical underpinnings is an additional layer to the self-learning offered through martial arts. Well-being is seen, then, as something that comes from the intellectual stimulation that the practise, and its understanding, affords. Through learning about the interconnection between mind, body and practice, a greater understanding of the self is possible. This reflective understanding supports self-control, particularly in managing circumstances that may have been more challenging beforehand, for example, in moments of anger or intimidation. 
Learning something new requires effort and perseverance. In a sense then, it is not just mastery of a practice but a mastery of the self that is also important. Learning discipline and control over the self can empower the shy, embolden the bullied and give a sense of physical confidence to those that do not feel this way usually. It is clear, then, that a greater connection and understanding of the self, self-mastery through self-discipline, perseverance - and the sense of accomplishment that comes from turning up to class and engaging regularly in practice - will, in a cyclical way, interact together to improve self-confidence and greater mental well-being.

Keeping the brain active, having an opportunity to learn something new and providing a way of life for those that chose it mean that martial arts are so much more than just a sport. The 21 st century is an era marked by a much greater focus on 'identity.' Identity when considered in conjunction with martial arts could be considered as important then, because the physical, external body offers both a sense and expression of the self. Thus, when we feel physically good and in charge of our physical selves, it stands to reason that we will experience ourselves more positively which inevitably will support our mental well-being.

When looking at physical health and mental well-being, a key theme to emerge as relevant and important was the notion of self-efficacy and selfmastery. In the preceding discussion, we have emphasised the importance of developing a sense of control over health and physical well-being - whether real or imagined. The emergence of well-being through mastery of practice, as well as the sense of general empowerment within everyday life that martial arts practice offers is made evident. However, whilst Bandura's use and understanding of the concept of self-efficacy in relation to health and well-being is useful, it is very much focused on the individual. As noted in Chapter 2, it places less emphasis on the role of the collective in supporting the development of the norms and values connected to practice, as well as the role of the teacher in facilitating the learning needed to develop martial arts practice. As Zhu Xi notes, learning and the consequent behaviour are the result of 'study, reflection, observation and practice' (Stanford Encyclopedia of philosophy, 2015: 15), none of which happen in a vacuum, devoid of a social context.

Before considering the social context of learning martial arts, it is worth making a brief note of the ways that martial arts were considered by the very many in this research to support the management of stress and depression. Whilst we again reiterate that we are making no causal claims ourselves as to the benefits in these specific areas, overwhelmingly, a resource to manage stress was noted. To try and unpick the precise aspect of the specific martial art practised that was considered as most valuable, and contributing most directly, is an impossible task. However, from the perspective of those that found benefit, the rationales offered were often myriad. Benefits that span the practical, the social and self-efficacy domains. In many ways, this could be seen as mirroring the multitude of 'stressors' within modern day life. Important to note once more though was that martial arts offered a vehicle to 
manage these stresses to some degree, reinforcing again the importance of ownership and control, not just over physical health and well-being but also over mental well-being.

\section{The social context of learning}

As we discussed earlier, human beings are intrinsically social and over time have come together to live in groups. If human beings are social, then it makes sense that their learned behaviour will be mediated through the social groups in which they live and spend time. Alongside the laws of the country of residence, behaviour is also shaped, modified and contained by the norms, values and expectations of the group in which one lives. Norms and values, as we have seen, are extremely important for holding groups together; they promote the social cohesion that is so important for societies and groups to function healthily.

However, the behaviours, values and norms expected of each group member are not biologically imprinted; instead, they must be learned through reinforcement and habitualisation. Ritualistic practices help embed the ideas and normalise them. At the macro level, we can see this at the level of nations and on a more micro, personal level, within friendship groups and families. There is, of course, a plethora of other types of groups, including groups that reflect shared interests and, in this case, groups such as martial arts. As a specific interest group, martial arts have their own sets of values, norms and expectations that are important to follow. Following the 'rules' ensures inclusion within the group, promoting a sense of belonging and shared identity that is the result of group membership. Martial arts then can be seen as serving two purposes, in terms of the health and well-being: (1) promoting the practices that support the physical health and well-being of individuals through practice, rituals and behaviour, and (2) providing a sense of community and belonging through club membership that is important for mental and social as well as mental well-being.

Social well-being is arguably an area of social life that is less considered particularly as it relates to health and mental well-being. As we have already mentioned, loneliness is one of the biggest issues facing people in the UK to date. This is an important point when looking at the impact of martial art practice on mental well-being, particularly as, overwhelmingly, we can see that having a network of support, friendships, community and a sense of belonging was a significant benefit of the martial arts practised by those included in the research. Social well-being and happiness were apparent for many of those who offered examples of how martial arts impacted on their everyday lives. Whilst finding friends was not necessarily an important aspect of learning a martial art for all of the participants, being part of a regular social group, meeting up with like-minded individuals and coming together to practise the same activity were important. Such practice appears to suggest 
that community and shared identity that both McNeill (1995) and Ehrenreich (2006) suggest is important. Coming together as a group to participate in a shared activity can create a sense of shared identity partly because of the shared rules and values associated with that activity. As sociologists such as Durkheim note, collective behaviour that is mirrored by the group through ritualistic movements is profoundly significant for cohesiveness and social bonding. In this instance then, we might suggest that a martial art is so much more than just a sport. It might be useful instead to consider it as a community who share practice that is martial arts, and not necessarily the other way around. One might argue that all physical activity is beneficial, so what is different about martial arts? From our research, we suggest that the broadest benefit appears to come from allowing for individual development whilst situating that individual within a broader community.

Indeed, belonging was a highly important benefit expressed by many participants in this research, where for some, lifelong friendships developed through the social dimension of participating regularly in martial art. Also mentioned frequently was the issue of trust. In this instance, trust developed through regular practice within a group of people who shared similar values and ideals, creating, as they did, a bond of social trust that is also highlighted in a wealth of literature as important for social well-being. As we noted earlier, the social dimensions of martial art practice infer a strong idea that coming together creates social bonds that promotes an almost transcendental type of well-being, a sense of well-being that is created via the community and connection. All of this is important for emotional and mental well-being. Thus, when we consider mental well-being, this research suggests that we should consider it, not just as related to needs that are very individualistic but, as approaches that also consider social components of mental well-being.

\section{The role of the teacher}

The role of the teacher is fundamental to the learning of anything and, in this case, to the learning of any martial art. When choosing a class, the teacher was clearly an important consideration. Interestingly, when seeking the 'right' teacher, personality and general character was less important to the student than the skill and experience of the teachers. Within this research, we can see a clear recognition that there is an evident distinction between skill and expertise and an ability to teach. As any academic will know, sometimes the greatest scholar is not always the best public orator and vice versa. The importance of professional over personal qualities, such as humour, is curious, given that these personal attributes help facilitate social interactions. Interestingly, and hearteningly, social demographics such as age and gender were also not considered relevant to a good teacher.

When considering the teacher within learning, data in this research also accentuated the need for authenticity in a martial art teacher. Students placed 
emphasis on having martial arts teachers that did not just know their art but lived their art, by following the expected behaviours and practices. It was important that their teachers had received good training themselves and engaged in regular training outside of their teaching, emphasising the importance of keeping up to date with their professional skills training development. The benefits of this could be inferred as twofold for student in that (1) it demonstrates an acknowledgement and understanding that learning is not static and fixed but fluid and on-going, and (2) students inevitably benefit most from teachers who are interested in their own learning and what they teach as well.

The notion of authenticity within teaching could also arguably be seen as linking to ideas of trust, an idea which was also highlighted as important in developing the relationships with teachers that were considered key to facilitating learning. Significantly then, whilst personal attributes were not important in the teacher as we have noted, the values and practices of the teacher were. In the case of martial arts, there was a clear and evident social dimension to the issue of trust, in that it connected very explicitly to attitudes and expectations around learning and teaching. For students, it was important that their teachers evidence encouragement and fairness within their teaching, and that all students were treated alike, and effort and hard work recognised. Indeed, encouragement proved to be an interesting aspect to the broader issue of motivation in learning. Positive reinforcement was considered as an enabler, enabling in that it supported an individual student's momentum to learn. Again, these ideas emphasise the importance of the social dimension of learning, in that the individual situates their own learning experiences within the community of learners they identify as belonging to. Remarkably, the importance of encouragement was indicated by male and females alike, as well as across the age ranges. This suggests that the need to have external stimuli in the form of motivation from others does not decline with age but retains a relevance that is of equal importance to all.

Authenticity was also considered important by martial arts teachers themselves, who made frequent references to it in both survey and interview data. Understanding about the cultures of the various martial arts taught, the language, the diet, lifestyles and history were considered as important contexts to the martial arts being taught. As Yu Gongbao notes, martial arts are not just a series of moves; instead, a martial art 'uses physical movements to express, expound and propagate the spirit of a culture...thus it is a physical form of...culture' (Siaw Voon Sim and Gaffney, 2018: 150). Martial arts are embedded in a history and a culture that is as valuable as the practice of the martial art itself. This is particularly true when considering the rules and norms around behaviour, etiquettes and hierarchy. Although not always foregrounded in the data from students of martial arts, confidence in the teachers' cultural and contextual knowledge and understanding was important for many. However, the desire for an authentic and embedded learning from teachers proved somewhat paradoxical to the teaching of students themselves. 
This is because the culture of martial arts teaching can be challenging for students who are used to the more interactive style of learning experienced in schools, or who are seeking more immediate results from their practice. The dojo - or teaching space - is very much an arena of experiential, didactic learning. A practice and form of teaching that has stood the test of time, persisting across the millennium, yet being in complete contrast to how education is experienced in the Northern Hemisphere.

\section{Identity in martial arts}

For some practitioners of martial arts - teacher or student - engaging in their practice was so much more than just learning a skill or technique. As noted earlier, martial arts provided a sense of community for some with belonging also offering a sense of a collective identity as well as one that was more centred on the self, in this case identity as a martial artist. For some respondents, this related to what they perceived a martial artist to be whilst for others it reflected a much more meaningful and profound understanding of who they are. What a martial artist is considered to be was very much focused on their attributes by the $62 \%$ who responded. Characteristics that related to the more instrumental qualities connected to the martial skill and attitudes to practice. Of those who responded, over three quarters were identified as martial artists to some degree, and, as we would expect, the longer the practice, the stronger the sense of this identity.

When considering identity construction within the realm of martial arts, it is interesting to note, although perhaps rather unsurprisingly, that a martial artist identity is something that is developed and reinforced over time. Time and practice could be considered as lending weight to a sense of an authentic martial arts self. At what point this identity moves beyond the dojo, transferring into the other arenas of everyday life, is not clear. Yet, this research also suggests that clearly for some, a martial arts identity was fundamental to how some individuals saw themselves. For some being a martial artist was their first and foremost sense of who they were. Understanding the ways that an identity can be fashioned through social interactions such as those found within activities such as martial arts suggests that identity is very much a social construction, as Berger and Luckman (1966) suggest. Under this premise, identity is not an intrinsic, embedded sense of self that is biologically determined. Instead, identity is something that develops through interactions with the environment and the people in it are, therefore, inherently social.

Whilst a discussion of identity within martial arts may not seem immediately obvious given that the focus of this book is on perceptions of health and well-being, martial arts do provide a valuable vehicle for understanding ways that individual respondents constructed identities for themselves within the space of their martial learning. The consequences in exploring a shifting sense of self is to make much more explicit the ways that self and self-identity are 
key to understanding attitudes, behaviours and practices that link to health and well-being. To illustrate, the disruptive, aggressive child - so labelled by their school, the woman who suffered domestic violence and felt powerless, to the short women afraid to walk alone. These are just some examples of individuals whose lives were transformed through the practice of a martial art. For these, martial arts enabled an ability to recreate a sense of self, a shift that contributed very significantly to areas of self-confidence, self-esteem and general well-being and, therefore, to lives they felt they were now living.

Thinking about identity in the context of martial arts is clearly relevant then, as it enables us to highlight the intricate ways that how we see ourselves impacts on our sense of self-efficacy, resilience and empowerment. It also highlights the important connection between mind and body and how these work together to influence, not only perceptions of health and well-being but also the sense of control we have over the practices that support these areas. Learning control over the mind and emotions is fundamental within martial arts, which, when transposed into other areas of our lives, is clearly valuable and significant. These ideas illuminate the socially constructed nature of individual beliefs about health and well-being. As we have seen through the empirical chapters, whether real or imagined, when beliefs change so does health and well-being, or at least the perception of health and well-being.

\section{Connecting communities and promoting health}

For the Asian philosopher Zhu Xi, self-awareness in both mind and body was the primary foundation block on which all other aspects of social life develop. As noted in Chapter 2, whilst he did believe that good governance was key to any society, he also felt that this should not be at the cost of personal development, the development which is supported through the direction and guidance of a teacher (Adler, 2014). In some respects, although no doubt not intended by Zhu $\mathrm{Xi}$ at the time of his writing, placing an emphasis on the 'self' for the learning he considered as important to personal development and growth is no less relevant today, than it was several thousand years ago, when he wrote these thoughts. Increasingly, at least when we think about health and well-being, responsibility for these areas has moved outside of the personal domain to that of the state. This is particularly true when we consider health, mental well-being and the support of these areas. Yet as we saw in Chapter 1, the monetary and social costs to the various governments around the world are phenomenal, in terms of supporting an increasingly ailing, ageing and stressed population. And, as we also note, this is not predicted to get any better; indeed, the opposite is true.

As numerous scholars note (Nettleton, 2013; Beattie, Gott, Jones and Sidell, 1993), health and well-being is very much framed within a discourse of Western notions of medicine, referred to as the biomedicine model. Prior to the formal establishment of medicine as a science, health was very much 
managed based on local customs, beliefs and religious and philosophical ideas. One could argue that the 21st century, at least in the West, is very much marked by a preoccupation with health and focus on the body (Crawford, 1984). This is indicative, as Nettleton (2013) notes, as we are much more likely to ask whether someone feels ill than whether they feel healthy.

In some respects, the ideas of $\mathrm{Zhu} \mathrm{Xi}$ appear to offer ancient solutions to our very modern problems. An emphasis and focus on self-learning will inevitably encourage a far greater sense of responsibility for our own health and well-being. Yet, this responsibility needs to be taught, from the very youngest of ages, so that occasions to learn and understand the connections between mind and body are embedded at the earliest opportunities. This research has suggested clearly that health and well-being can very much be considered in terms of qualitative understandings, which come through exposure to opportunities to learn. This is transparently via the subjective perceptions and beliefs; such insight would not have been possible within more positivistic approaches to measuring and quantifying health.

In this research, Bandura's (1998) ideas of self-efficacy can very much be seen as evidenced in the ways that individuals construct their identities, developing confidence and a sense of personal empowerment, as they perceive progress and mastery over their art. Yet whilst this learning is individual, it is not individualistic; instead, it occurs within a social context, where community, belonging and a sense of identity feed very much into the development of resilience and efficacy. If, as this research suggests, with increased confidence comes shifting identities, this would also suggest that community is very important for constructing, not just to which group one belongs, as in this context, but also who one is. As is clearly evidenced here, martial arts practice was perceived to offer profound benefits for many of those that took part in this research. Either in managing or improving specific health and physical conditions or as a means of coping with difficult and stressful lives, martial arts practice offered an approach to dealing with these areas that allowed individuals to feel empowered and to feel that they had some control over their own health and well-being. In this sense then, one might argue that by connecting people together through communities of practice, health and well-being improve. Connecting communities together is more than just bringing people together; however, it is also about connecting communities of practice across time and space, linking cultures and philosophies so that the emergent identities are framed within practices that are not parochial but fluid, in relation to the influences upon them. Health and well-being, in the context of this research on martial arts practice, suggest that the domain of health and well-being could be usefully re-conceptualised then, to be considered as something that is clearly situated within the social domain.

Whilst not a specific aspect of this research, it is still worth mentioning the additional ways that the social domain of learning can impact on health and well-being, in terms of access and resources. As we saw in Chapter 4, 
having access to good quality classes, as well as being able to afford to attend them is important. Thus, the location of classes, the cost of tuition, clothing and equipment can be barriers for some. Whilst we did not explore socioeconomic background in this research, we do know that social location, in terms of socio-economic status, will have a bearing on the availability of money to cover costs associated with different sporting activities. This is ironic given that, as we saw earlier, the rates of access to medication and other lifestyle practices are the lowest for those who are also most at risk of some diseases and illnesses, that is, the poorest. As Bourdieu (1997) notes, exposure to different ideas and practices is also important for developing a taste and preference for them. This means that it is important to ensure that all people, regardless of age and circumstances, should have the opportunity to access these sorts of activities. Not only would this support the development of awareness that Zhu Xi mentions as important, it will also help support the much greater ownership of health and well-being. In addition, in this research, many participants suffered from illnesses and conditions that had a profound and significant impact on their general quality of life; yet, they found support and relief for these through their martial arts practice. In managing the demands of multitude of medical conditions that are bombarding health systems around the world because of the sheer volume, it makes sense then that an economic investment to ensure access to activities such as martial arts would provide a means of support to both the individuals and the health organisation themselves.

\section{Chapter summary}

In this chapter, we have drawn together the key themes from our research, a study of 511 participants, participants. The participants in this research encompassed the full range of ages, experiences of martial arts practice and the types of martial arts practised. It sought to explore the role of martial arts in shaping perceptions of health and well-being. The data have clearly shown that martial arts are considered as contributing in positive ways to these and physical benefits and to the benefits that connect to mental well-being. The ways that practice, beliefs and outcomes interconnect were also considered in relation to a range of theoretical ideas, with the data strongly supporting the socially constructed nature of health and well-being. The implications of these findings will be considered in the final chapter. 


\section{Chapter 7}

\section{Conclusion}

In this concluding chapter, we draw together the key ideas presented in the book, focusing specifically on how the practice of martial arts, at whatever level of expertise, can contribute in important ways to health and well-being. Before doing this, it is important to re-situate the rationale for our research focus within its broader context. As highlighted in Chapters 1 and 2, the new millennium is the one that is marked by an impending catastrophe in terms of global health and well-being. As we also noted, there is a vast array of factors that explain the reasons for this imminent crisis, as well as how best to manage the more immediate concerns, most specifically in terms of prevention. We discussed how many of the illnesses currently straining health services around the world are closely connected to lifestyle, for example, Type 2 diabetes and obesity as well as other lifestyle practices, such as smoking, alcohol consumption and sedentary lifestyles. Alongside specific health challenges, we also drew attention to the growing concerns connected to the area of mental well-being, concerns that span the continuum of ages, from the young to the very old. Mental ill health, such as depression and other chronic conditions, as well as stress, anxiety and loneliness are unprecedented in terms of the numbers of people struggling in these areas.

Given the growing global concern about health and well-being, as well as worries as to the increasing demand on the resources required to tackle these, it seemed pertinent to ask whether there were alternative ways to offer cost-effective sources of support, at least to some of these areas. Given that the UK National Health Service (2017) states that those who undertake no physical exercise are twice as likely to suffer a heart attack as those that do, a focus on physical activity was relevant. With this in mind, we considered the following research questions:

- How and in what way can martial arts be considered as contributing to health and well-being from the perspective of those that practise them?

- Do martial arts impact on everyday lifestyles and practices and, if so, how, from the perspective of those that practise it? 
By exploring the ways that engaging in martial arts can inform individual narratives of self, specifically - and in the context of this research - as relates to individual perceptions of one's own health and well-being. The primary aims of this research were, therefore, to understand and explain notions of health and well-being, specifically health and well-being as constructed through practice, alongside how these notions can be subject to change. In light of these aims, the key objective framing the research was to better understand how perceptions and beliefs impact on everyday life and how, and in what way, from the viewpoint of those that experience these impacts, they relate to perceived gains to health and well-being? The aims and objectives of the research have a clear rationale for its undertaking. Put simply, recognising and highlighting the link between practice, beliefs and outcomes have important consequences and implications for health practitioners, as well as the more generalised support of health and well-being.

Merely presenting the beliefs surrounding martial arts, health and wellbeing in a simple and descriptive way offers no real explanatory power without a consideration of the role of theory in making sense of these beliefs. Narratives associated with perceptions of health and well-being need to be considered within a theoretical framework. This is because the use of theory to explain behaviour is like providing a means of illumination, suggestive of a window through which we can look. Framing the data we collected within different ideas to explain it enabled us to make sense of the data. Specifically, theory in this research enabled us to see and explain how beliefs are shaped through practice, reinforced and then reproduced through that practice. Such knowledge is important if the research is to be meaningful.

To address the question then, of how and in what way, martial arts be considered as contributing to health and well-being from the perspectives of those that practise them, the data in this research suggest that these practices do indeed contribute to feeling healthier both physically and mentally, at least from the perspective of the participants in this research. Explaining the 'how' and 'in what way,' however, is far from straightforward. In terms of the 'how,' data in this research suggest that practice and the repetition of that practice directly impact on very obvious areas, areas that one would expect to see changes in when engaged in regular sporting activities such as martial arts, and areas such as flexibility, balance and mobility. A focus on posture within martial arts, for example, will inevitably lead to improvements in issues connected to joints and balance. Support for other more serious and challenging health conditions, such as arthritis and asthma, can also be understood as finding relief from the particular techniques of different martial art practice. The 'how' can also be seen as a reflection of the commitment to practise and its regularity, as well as the focus on what one is striving to learn. Commitment and regularity of practice contribute to a sense of positive motivation and achievement. Therefore, the how and in what way martial arts can be considered as contributing to health and well-being can be understood as 
a response to the applied and direct consequences of physical activity - the self-fulfilling prophecy discussed in Chapter 6. It is also explained as a result of the more indirect, transcendental benefits that come when one focuses and strives to achieve and learn something, and where personal progress, however small, is experienced.

As is well evidenced in the data, the benefits of martial arts practice also extended beyond the physical and encompassed benefits to mental well-being too. Overwhelmingly, practice offered support for managing stress and anxiety, and, by association, the health conditions that are connected to these areas, such as high blood pressure and headaches. A significant conclusion drawn from our data is that a sense of self-mastery is key to explaining the benefits that are perceived as resulting from martial arts practice. Self-mastery in this research refers to a sense of resilience and self-efficacy in managing and dealing with issues connected to health and well-being. It also refers to mastery over the self, as opposed to the self-finding mastery in the martial art. Through perseverance with practice, improvements are noted, either as a direct consequence of the physical practice or because of the application of techniques and skills taught, outside of the dojo.

Interestingly, the results of this research also suggest an important emphasis on the social dimension to learning, which included having opportunities to watch and learn from role models, that is, experienced and knowledgeable teachers, as well as from group members in which learning takes place and is shared. The group aspect of martial arts was a key aspect of the ways that martial arts could be considered as contributing to health and wellbeing, specifically within the domain of mental well-being. 'Belonging' and 'collective practice' were highlighted as important in promoting a sense of shared identity, as well as a more reflexivity defined identity that key to how an individual saw them self. A community of practice offered a source of companionship, friendship and support for some, as well as a routine and structure to the week for those that felt isolated or needed something more in their everyday lives.

To address the question of whether martial arts impact on everyday lifestyles and practices and if so how, the data in this research strongly support the assertion that the impacts for some were really quite profound. A greater connection to the physical self, alongside a much greater awareness of how the mind and the body interconnect, resulted in some significant changes in day-to-day living for many of those included, for example, much greater awareness of the importance of healthier lifestyle practices, such as less sugar and better posture, as well as the cessation of habits that are damaging, such as smoking. Simple things, such as feeling able to manage anxiety and stress, to recognising the importance of sleep to mental well-being and energy levels indicate very clearly how important a degree of ownership is in promoting health and well-being. Again, this highlights the idea of self-mastery, where such mastery empowers with essential consequences. 


\section{Significance of the research}

The data from this research suggest that martial arts are a wonderful vehicle for promoting health and well-being as well as supporting lifelong learning. It is also an important resource for connecting communities, promoting cultural awareness and offering self-empowerment for those who seek it. Of course, that is not to say that martial arts are the only medium through which such benefits can be achieved. That said, martial arts do arguably offer something a little more bespoke than a standard sport. This assertion is based on the fact that most martial arts practice is swathed in philosophy and has customs, etiquettes, norms and rules that are not so for many other sports. This research has, therefore, allowed us an interesting glimpse into the nature of martial arts practice, as well as a small degree of understanding as to how the benefits and consequences of martial arts practice are perceived by those that both teach and are taught. In terms of focus and design, this research is, therefore, the first of its kind, in terms of both the scale and data collection techniques. As noted earlier, most studies of the benefits of martial arts tend to be small scale and qualitative. This research surveyed more than 500 practitioners of martial arts and interviewed 40. Data were collected from across the spectrum of age and level of skill and includes an international flavour within the data. Whilst the snowballing, convenience sampling means that it is impossible to generalise from the data collected, and mindful of the potential positive bias amongst the respondents who took part, this research still makes a meaningful and significant contribution to the fields of martial arts, sports and health and well-being.

Human beings are intrinsically social and their understanding of themselves comes from their social interactions and understandings of how they fit with particular groups and within society. This study, with its focus on martial arts, has offered a snapshot which has enabled us to see very clearly the socially constructed nature of individual identities, as related to sense of self as well as beliefs and how they emerge - in this case as related to health and well-being. It has also offered a window into the profoundly social nature of the way both our behaviour and beliefs are shaped. Understanding what people do and believe in relation to health and well-being practice is important if the health and mental well-being challenges of the new millennium are to be addressed. This research is, therefore, also both innovative and original in the way it has framed the issues, drawing on a range of theories, and sought to take a bottom-up approach in attempting to make sense of this important social issue and concern.

In summary, this research is significant on a number of levels and makes a clear and valuable contribution to both our understanding of issues of health and well-being and the theory that seeks to explain it. Through a unique and original research design, insights have been offered to make sense of what people think about their health and well-being, the connection with 
their martial arts practice and why they think what they do as well as how these beliefs manifest in day-to-day lives. In this research, martial arts were also used as an interesting medium to explore and consider the applicability of different theoretical frameworks to make sense of the data that emerged, thus contributing significantly to our understandings in this area. In tandem to this, an approach that combines psychological, sociological and philosophical theories highlights very clearly the value of taking a multi-disciplinary approach a multi-disciplinary social issue. As $\mathrm{Zhu} \mathrm{Xi}$ notes, a holistic approach is needed when studying the physical world (Stanford Encyclopedia of Philosophy, 2015). A holistic approach that takes account of the social, cultural and historical contexts as well as self-observations and reflections is key - it is a multitude of these selves that make up society after all.

\section{Recommendations for policy and practice}

Emerging from the data collected through this research are a number of recommendations for policy and practice as relates to the importance of life-long learning. We offer these as suggestions:

1 A clear finding as related to the impact of martial arts practice on health and well-being was the notion of experiential learning, that is learning through experience. This suggests that when attempting to tackle issues connected to health and well-being, it is not enough to simply tell someone what they must do to change. Change comes from authentic opportunities to experience and to then learn from those experiences. Such experiences also need to be reinforced over time, through their repetition. Therefore, in tackling health and well-being issues connected to lifestyle choices and practices, exposure and experiences matter and only then are we likely to create in-roads into tackling the changes in behaviour that are needed. An advert or a flyer is not going to do it.

2 In much the same way, the benefits to management issues connected to mental well-being need to be made much more apparent and clearer. Martial arts are most often perceived as a form of self-defence training, meaning that the other positive aspects connected to the practice can be missed by those who have no other exposure. For example, martial arts are often assumed to be unsuitable for the emotionally volatile child; yet, the exact opposite is true. In this research, martial arts facilitated the learning of control over emotions, reflecting its value in this area. Thus, a broader awareness raising as to the value of martial arts, beyond the stereotypical, would be valuable and would also mean likely that more people would be willing to give it a go, beyond those that chance upon it serendipitously.

3 It is important that children from the earliest ages are taught to manage their health and mental well-being and be given tools to help support the 
development of their mastery of this area. In addressing this area at an early stage, there is a strong chance that at least some children will benefit longer term, and into adulthood.

4 Access to martial arts is patchy and erratic, with some locations having multiple teachers and martial arts schools and some having very few or none. In addition, affordable spaces in which to take classes are not always readily available and so can therefore be cost prohibitive. Some of the smaller classes run for specific groups such as the elderly, and patients in mental health hospital or groups with particular needs do not always generate enough income to cover costs, let alone make a living for the teacher. It is important then that resources are made available to cover the costs of delivering these sessions to particular vulnerable groups. Without it, it is likely that these groups will eventually disappear. Not only do these provide a valuable service, but they are often also a life line to the lonely and isolated. Their value is, therefore, myriad and extends beyond a mere exercise class.

5 Not all families can afford the weekly class costs, and costs for expenditure on insurance, gradings, clothing and travel to competitions. Given the ways that martial arts have been highlighted in this research as significant to boosting confidence and self-esteem of the many included, it would make sense then to offer martial arts as part of the national curriculum. Funds to cover it could usefully be redirected from monies that are provided by governments to support the socially disadvantaged, Pupil Premium payments for Free School meals children in the United Kingdom, as an example.

6 In research that explores behaviour and practice within the domain of health and well-being, it is perhaps useful to move beyond thinking of health and well-being in either medical or social terms and instead approach research in this area as a much more holistic phenomenon, where the physical and social worlds are considered so interwoven and entwined that to consider one without the other would be unthinkable. 


\section{References}

Adler, J.A., (2014), Reconstructing the Confucian Dao: Zhu Xi's Appropriation of Zhou Dunyi, New York: State University of New York Press.

Ashworth, E.A. and Rhodes, P., (2017), History of Medicine, Encyclopaedia Britannica, www.britannica.com/science/history-of-medicine (accessed July 2019).

Babbie, E., (2002), The Basics of Social Research, Belmont: Wadsworth Thomson Learning.

Bandura, A.; Ross, D. and Ross, S.A., (1961), Transmission of Aggression through Imitation of Aggressive Models, Journal of Abnormal and Social Psychology, 63: 575-582.

Bandura, A., (1994), Self-efficacy, in Ramachaudran, V.S., (Ed.), Encyclopaedia of Human Behaviour, Vol. 2, New York: Academic Press, pp. 71-81.

Bandura, A., (1997), Exercise of Personal and Collective Efficacy in Changing Societies, in Bandura, A, (Ed.), Self-efficacy in Changing Societies, Cambridge: Cambridge University Press, pp. 1-45.

Bandura, A., (1998), Health Promotion from the Perspective of Social Cognitive Theory, Psychology and Health, 13: 623-649.

Beattie, A.; Gott, M.; Jones, L. and Sidell, M., (1993), (Eds) Health and Wellbeing: A Reader, Basingstoke: Open University Press.

Beck, U., (1992), Risk Society: Towards a New Modernity, London: Sage.

Bell, C., (2008), Asian Martial Arts and Resiliency, Ethnicity and Inequalities in Health and Social Care, 1(2): 11-18.

Berger, P. and Luckmann, T., (1966), The Social Construction of Reality: A Treatise in the Sociology of Knowledge, London: Penguin.

The British Arts Council, (2014), The Value of Arts and Culture to People and Society: An Evidence Review, British Arts Council, pp. 1-54.

Bourdieu, P., (1997), The Forms of Capital, in Halsey, A.H.; Lauder, H.; Brown, P.; Wells, S., (Eds), Education, Culture, Economy, Society, Oxford: Oxford University Press, pp. 46-59.

Burke, D.T.; Al-Adwal, S.; Lee, Y.T. and Audetter, J., (2007), Martial Arts as Sport and Therapy, The Journal of Sports Medicine and Physical Fitness, 47: 96-102.

Chandola, T., (2010), Stress at Work, British Academy Report, pp. 1-104.

Chapman, S. and Rayner, G., (2012), Should Celebrities Get Involved in Public Health Campaigns? BMJ, www.bmj.com/press-releases/2012/09/25/shouldcelebrities-get-involved-public-health-campaigns (accessed August 2017). 
Collins, R., (2004), Interaction Ritual Chains, Princeton: Princeton University Press.

Crawford, R., (1984), A Cultural Account of 'Health': Control, Release and the Social Body, in Beattie, A.; Gott, M.; Jones, L. and Sidell, M., (1993), Health and Wellbeing: A Reader, Basingstoke: Open University Press, pp. 41-47.

Cresswell, J.W. and Piano-Clark, V.L., (2007), Designing and Conducting Mixed Methods Research, London: Sage.

Cynarski, W.J., (2017), The Philosophy of Martial Arts: The Example of the Concept if Ido, AUC Kianthropolica, 53(2): 95-106.

Department for Health, (2012), No Health without Mental Health: Implementation Framework, Her Majesty's Government, pp. 1-54.

Durkheim, E., (1995), The Elementary Forms of Religious Life, New York: The Free Press.

Durkheim, E., (1997), The Division of Labour in Society, New York: The Free Press.

Dutta, S. and Marquez, P.V., (2012), Cervical Cancer Undermines Gender Equality in Africa, The World Bank, http://blogs.worldbank.org/africacan/cervical-cancerundermines-gender-equality-in-africa (accessed August 2017).

Dweck, C., (2000), Self-theories: Their Role in Motivation, Personality and Development, New York: Psychology Press.

Ehrenreich, B., (2006), Dancing in the Street: A History of Collective Joy, New York: Metropolitan Books.

English Oxford Dictionary, (2017), https://www.lexico.com/en/definition/culture

Escoto, K.H.; Laska, M.N.; Larson, N.; Neumark-Sztainer, D. and Hannan, P.J., (2012), Work Hours and Perceived Time Barriers to Healthful Eating Among Young Adults, American Journal of Health Behavior, 36(6): 786-796.

European Committee for Social Cohesion, (2004), A New Strategy for Social Cohesion, Council of Europe, pp. 1-28.

The GBD 2015 Obesity Collaborators, (2017), Health Effects of Overweight and Obesity in 195 Countries over 25 Years, New England Journal of Medicine, 377: $13-27$.

Goodger, J., (1986), Ritual Solidarity and Sport, Acta Sociologica, 29(3): 219-224.

Graham, H., (1984), Women, Health and the Family, Brighton: Harvester Wheatsheaf.

Heart UK, Coronary Heart Disease remains the Number 1 killer in the UK, https:// heartuk.org.uk/press/press-kit/key-facts-figures (accessed May 2017).

Hex, N.; Bartlett, C.; Wright, D.; Taylor, M. and Varley, D., (2012), Estimating the Current and Future Costs of Type 1 and Type 2 Diabetes in the UK, Including Direct Health Costs and Indirect Societal and Productivity Costs, Diabetic Medicine, 29(7): 855-862.

Hopton, A.K.; Curnoe, S.; Kanaan M. and MacPherson, H., (2012), Acupuncture in Practice: Mapping the Providers, the Patients and the Settings in a National Cross-Sectional Survey, BMJ: Open Access, 2(1): e000456.

Houri, D.; Nam, E.W.; Choe, E.H.; Min, L.Z. and Matsumoto, K., (2012), The Mental Health of Adolescent School Children: A Comparison Among Japan, Korea, and China, Global Health Promotion, 19(3): 32-41.

Hu, F.B., (2011), Globalization of Diabetes: The Role of Diet, Lifestyle, and Genes, Diabetes Care, 34(6): 1249-1257.

Hwang, H.F.; Chen, S.Y.; Lee-Hsieh, J.; Chein, D.K.; Chen, C.Y. and Lin, M.R., (2016), Effects of Home-Based Tai Chi and Lower Extremity Training and Self-Practice on Falls and Functional Outcomes in Older Fallers from the Emergency Department - A Randomised Control Trial, Journal of the American Geriatrics Society, 64(1): 518-525. 
Insel, T., (2015), Numbers, The National Institute for Mental Health, www.nimh. nih.gov/about/directors/thomas-insel/blog/2015/mental-health-awarenessmonth-by-the-numbers.shtml (accessed July 2017).

International Diabetes Federation, (2015), IDF Diabetes Atlas, Seventh Edition, IDF, pp. 1-140.

Jones, D. and He, J., (2015), Returning to Zhu Xi: Emerging Patterns within the Supreme Polarity, New York: SUNY Press.

Kosenko, K.A.; Binder, A.R. and Hurley, R., (2016), Celebrity Influence and Identification: A Test of the Angelina Effect, Journal of Health Communication; International Perspectives, 21(3): 318-326.

Krippendorff, K., (2004), Content Analysis: An Introduction to Its Methodology (Second Edition), Thousand Oaks: Sage.

Kvale, S. and Brinkmann, B., (2009), Interviews: Learning the Craft of Qualitative Research Interviewing, Los Angeles: Sage.

The Lancelet Commission, (2014), Culture and Health, The Lancelet, 384: 1607-1639.

Leisinger, K.M.; Gorabedian, L.F. and Wagner, A.K., (2012), Improving Access to Medicines in Low and Middle Income Countries: Corporate Responsibilities in Context, Southern Med Review, 5(2): 3-8.

Leisure DB, (2018), State of the UK Fitness Industry Report, www.leisuredb.com/ blog/2018/5/16/2018-state-of-the-uk-fitness-industry-report-out-now (accessed November 2018).

Levitin, D., (2014), The Organised Mind: Thinking Straight in the Age of Information Overload, New York: Penguin.

Lincoln, Y.S. and Guba, E.G., (1985), Naturalistic Inquiry. Newbury Park: Sage Publications.

Liu, H.; Yeh, C.K.; Chick, G.E. and Zinn, H.C., (2008), An Exploration of Meanings of Leisure: A Chinese Perspective, Leisure Sciences, 30(5): 482-488.

Loignon, C.; Hudon, C.; Goulet, E.; Boyer, S.; De Laat, M.; Fournier, N.; Grabovschi, C. and Bush, P. (2015), Perceived Barriers to Healthcare for Persons Living in Poverty in Quebec, Canada: The EQUIhealThY Project, International Journal for Equity in Health, 14(4): 1-11.

Luppino F.S.; de Wit, L.M.; Bouvy, P.F.; Stijnen, T.; Cuijpers, P.; Penninx, B.W.J.H. and Zitman, F.G., (2010), Overweight, Obesity, and Depression: A Systematic Review and Meta-analysis of Longitudinal Studies, Arch Gen Psychiatry, 67(3): 220-229.

Matchim, Y. and Armer, J., (2007), Measuring the Psychological Impact of Mindfulness Meditation on Health among Patients with Cancer: A Literature Review, Oncology Nursing Forum, 34(5): 1059-1066.

Matusiewicz, A.K.; Hopwood, C.J.; Banducci, A.N. and Lejuez, C.N., (2011), The Effectiveness of Cognitive Behavioral Therapy for Personality Disorders, Psychiatric Clinics of North America, 33(3): 657-685.

McHugh, K.R.; Hearon, B.A. and Otto, M.W., (2010), Cognitive Behavioral Therapy for Substance Use Disorders, Psychiatric Clinics of North America, 33(3): 511-525.

McLeod, S., (2007), Skinner: Operant Conditioning, Simply Psychology, www. simplypsychology.org/operant-conditioning.html (accessed August 2017).

McLeod, S., (2016), Bandura: Social Learning Theory, Simply Psychology, www. simplypsychology.org/bandura.html (accessed August 2017). 
McNeill, W.H., (1995), Keeping Together in Time: Dance and Drill in Human History, Cambridge: Harvard University Press.

Minkler, M., (1999), Personal Responsibility for Health? A Review of the Arguments and the Evidence at Century's End, Health, Education and Behaviour, 26(1): 121-141.

Mortimer, J.A.; Ding, D.; Borenstein, A.R.; DeCarli, C.; Guo, Q.; Wu, Y.; Zhao, Q. and Chu, S., (2012), Changes in Brain Volume and Cognition in a Randomized Trial of Exercise and Social Interaction in a Community-Based Sample of Non-Demented Chinese Elders, Journal of Alzheimer's Disease, 30(4): 757-766.

Nail, P., (1998), Behaviourism and a Theory of Personality: A Critical Look, The SAPA Project, Northwestern University, www.personalityresearch.org/papers/naik. html (accessed June 2017).

National Centre for Health Statistics, (2014), Depression in the U.S. Household Population 2009-2012, NCHS Data Brief No. 172: 1-8.

National Centre for Health Statistics, (2017), Wireless Substitution: Early Release of Estimates From the National Health Interview Survey, July-December 2017, https:// www.cdc.gov/nchs/data/nhis/earlyrelease/wireless201806.pdf

National Health Service Choices, (2017), Coronary Heart Disease; Prevention, www. nhs.uk/Conditions/Coronary-heart-disease/Pages/Prevention.aspx (accessed July 2017).

National Institute of Aging and National Institutes for Health, (2007), Why Population Aging Matters: Global Perspectives, US Department of Health, pp. 1-32.

National Institute of Diabetes and Digestive and Kidney Diseases, (2016), Symptom and Causes of Diabetes, www.niddk.nih.gov/health-information/diabetes/ overview/symptoms-causes (accessed July 2017).

National Institute for Health, (2015), Trends in the Use of Complementary Health Approaches Among Adults: United States, 2002-2012, National Health Statistics Report, pp. 1-16.

Nettleton, S., (2013), The Sociology of Health and Illness, Third Edition, Cambridge: Polity.

Office for National Statistics, (2009), The Health Statistics Quarterly 38, ONS.

Office for National Statistics, (2015), Harmonised Concepts and Questions for Social Data Sources: Personal Well-Being, Crown Copyright, pp. 1-6.

Organisation for Economic Co-operation and Development, (2017), Social Cohesion, www.oecd.org/dev/inclusivesocietiesanddevelopment/social-cohesion.htm (accessed July 2017).

Park, J.; Linde, K.; Manheimer, E.; Albrecht, M.; Sherman, K.; Smith, C.; Sung, J.; Vickers, A. and Schnyer, R., (2008), The Status and Future of Acupuncture Clinical Research, The Journal of Alternative and Complementary Medicine, 14(7): 871-881.

Pons van Dijk, G.; Leffers, P. and Lodder, J., (2014), The Effectiveness of Hard Martial Arts in People over Forty: An Attempted Systematic Review, Societies, 4: 161-179.

Roth, G.; Forouzanfar, M.; Moran, A.; Barber, R.; Nguyen, G.; Feigin, V.; Naghavi, M.; Mensah, G. and Murray, C., (2015), Demographic and Epidemiologic Drivers of Global Cardiovascular Mortality, New England Journal of Medicine, 372: 1333-1341.

Schwarzer, R. and Fuchs, R., (1997), Changing Risk Behaviours and Adopting Health Behaviours: The Role of Self-efficacy Beliefs, in Bandura, A., (Ed.), Selfefficacy in Changing Societies, Cambridge: Cambridge University Press, p. 288. 
Seguin, R.; Connor, L.; Nelson, M.; LaCroix, A. and Eldridge, G., (2014), Understanding Barriers and Facilitators to Healthy Eating and Active Living in Rural Communities, Journal of Nutrition and Metabolism, 2014: 1-8.

Setton, M., (2008), in Ochieng'- Odhiambo, F.; Burton, R. and Brandon, E., (Eds), Conversations in Philosophy, Crossing the Boundaries, Newcastle: Cambridge Scholars Press, pp. 56-61.

Sharma, M. and Haider, T., (2015), Tai Chi and an Alternative and Complimentary Therapy for Anxiety: A Systematic Review, Journal of Evidence Based Complementary Alternative Medicine, 20(2): 143-153.

Siaw Voon Sim, D. and Gaffney, D., (2018), Chen Taijiquan: Masters and Methods, Columbia, WA: D and B Publications.

Skinner, B.F., (1953), The Analysis of Behaviour, New York: The Macmillan Company.

Stanford Encyclopedia of Philosophy, (2015), Zhu Xi, https://plato.stanford.edu/ entries/zhu-xi/ (downloaded 2017).

Stevenson, D. and Farmer, P., (2017), Thriving at Work: The Independent Review of Mental Health and Employers, https://assets.publishing.service.gov. uk/government/uploads/system/uploads/attachment_data/file/658145/thrivingat-work-stevenson-farmer-review.pdf (accessed 2018).

The Telegraph, (2017), Number of Vegans in Britain Rises by 360\% in 10 Years, www.telegraph.co.uk/food-and-drink/news/number-of-vegans-in-britain-risesby-360-in-10-years/ (accessed August 2017).

Thorogood, N., (2002), What Is the Relevance of Sociology to Health Promotion? in Brunton, R. and Macdonald, G., (Eds), Health Promotion: Disciplines, Diversity and Development, London: Routledge, pp. 1-21.

University of Berkley, (2019), Mind-Body Exercise: Tai Chi and Yoga, Berkeley Wellness, www.berkeleywellness.com/healthy-mind/mind-body/article/mindbody-exercise-tai-chi-and-yoga (accessed January 2019).

Viruás-Oretega, J., (2006), The Case Against B.F. Skinner 45 Years Later: An Encounter with N. Chomsky, Behaviour Analyst, 29(2): 243-251.

Wang, J. and Stringer, L.A., (2000), The Impact of Taoism on Chinese Leisure, World Leisure Journal, 42(3): 33-41.

Wang, F.; Lee, E.K.; Wu, T.; Benson, H.; Fricchione, G.; Wang, W. and Yeung, A.S., (2014), The Effects of Tai Chi on Depression, Anxiety and Psychological WellBeing: A Systematic Review and Meta-Analysis, International Journal of Behavioural Medicine, 21(4): 605-617.

Witte, K. and Böckelmann, I., (2016), Comparing the Effectiveness of Karate and Fitness Training on Cognitive Functioning in Older Adults - A Randomized Control Trial, Journal of Sport and Health Science, 5(4): 484-490.

Wong, E., (2015), Being Taoist: Wisdom for Living a Balanced Life, Boston: Shambhala Publications.

World Health Organisation, (2006), BMI Classification: Global Database on Body Mass Index, WHO, http://apps.who.int/bmi/index.jsp?introPage=intro_3.html (accessed June 2017).

World Health Organisation, (2012), Prevalence of Mental Disorders, www.euro.who. int/en/health-topics/noncommunicable-diseases/mental-health/data-andstatistics (accessed July 2017).

World Health Organisation, (2014), World Health Statistics 2014, https://apps.who. int/iris/bitstream/handle/10665/112738/9789240692671_eng.pdf;jsessionid= F45BDA6E415F21C31780264DEC4188D0?sequence $=1$ 
World Health Organisation, (2016), Global Report on Diabetes, WHO, pp. 1-88.

World Health Organisation, (2017a), Ten Facts on Global Health, WHO, www.who. int/features/factfiles/global_burden/en/ (accessed June 2017).

World Health Organisation, (2017b), WHO Global Health Days, www.who.int/ campaigns/en/ (accessed August 2017).

Yu, P. and Berryman, D.L., (1996), The Relationship among Self-Esteem, Acculturation, and Recreation Participation of Recently Arrived Chinese Immigrant Adolescents, Journal of Leisure Research, 28(4): 251-271.

Zen and Buddhism, (2019), Zen and Martial Arts, www.zen-buddhism.net/martialarts/zen-and-martial-arts.html (accessed January 2019). 


\section{Index}

Note: Bold page numbers refer to tables and page numbers followed by " $n$ " denote endnotes.

acupuncture 4

alternative treatments: acupuncture 4; and well-being 4

Armer, J. 23

attention-deficit hyperactivity disorder (ADHD) 14

attitudes: and behaviour 15; individual 13-14

Bandura, A. 15-18, 87, 90, 92-93, 99

Beck, U. 20

behaviour 14-15; and attitudes 15 ; collective 21; individual 13-14; social cognitive theory $15-16$; and society $18-20$

behaviourism 14-15, 84, 90

Bell, C. 23

Berger, P. 19, 97

Berryman, D.L. 23

'Bobo doll' experiment 15

body mass index 2

Book of Changes 22

Bourdieu, P. 19, 100

British Arts Council 4

British Medical Journal 4

British Office for National Statistics 7, 34

Buddhism 23, 72, 85

'Bushido' 23

Carradine, David 31, 87

Ch'an (Zen) Buddhism 22

Chandola, T. 3

Chick, G.E. 23

China: and diabetes 2; and Eastern philosophy 22-23; and martial arts 22-23; Tai Chi 24-25; Taoism 22-23
Chinese culture 71

chronic heart disease: deaths from 1; and United Kingdom 1; and United States 1 collective behaviour 21

collective identity 97

communities: belonging 73-74; connecting, and promoting health 98-100; and martial arts 37-39

conditioned behaviour see operant conditioning

confidence 65 ; in martial arts 66-69; physical 66-67, 78, 82; well-being and 66-69

Confucianism 23, 85

Confucius 25

Council of Europe 19

Cresswell, J.W. 7

culture 85; Chinese 71; defined 19, 20; and ethics 71-72; and group behaviour 20-21; and health 13; of learning 70; and martial arts 37 ; well-being 13 , $71-72$

Curnoe, S. 4

Cynarski, W.J. 22

Daoism 22, 24

diabetes 1-2, 11n1; and China 2; and well-being 2

discipline 67-69; mental 68, 78; well-being and 67-69

Durkheim, E. 20, 95

Dweck, C. 18

Eastern philosophy 85; and China 22-23; and martial arts 22-26

Eating and Activity Survey 13 
eating habits: and economic resources 19 economic resources: and eating habits 19; and health 20; and lifestyle practices 19-20; and tastes 19; see also poverty

Ehrenreich, B. 21, 95

ethics and well-being 71-72

European Union 3

exercise: and health 1; and well-being 1

Fuchs, R. 18, 87, 92

Goodger, J. 21

Graham, H. 89

group behaviour: and culture 20-21; and well-being 21; see also behaviour

The Guardian 3

Guba, E.G. 11

habitualisation 19

health: connecting communities and promoting 98-100; and culture 13; and economic resources 20; and exercise 1 ; management of stress and the link to 75-82; and martial arts 1,21-22, $28-29,32-34,52-57, \mathbf{5 3}$; and poverty 13, 20; and self-efficacy 17-18

health awareness 89-91; and martial arts 50-52, 51; and martial arts teachers 32-33, 32-34

health benefits $88-89$

Hopton, A.K. 4

Hu, F. B. 2

identity: collective 97; in martial arts 97-98; shared 94-95, 103; social 85 individual attitudes 13-14 individual behaviour 13-14 intellectual 65; defined 69; well-being 69-72

International Diabetes Federation (IDF) 2

Japanese martial arts 23

Kanaan M. 4

Kung Fu 24

'law of effect' 14

learning: challenges in martial arts 61-62; martial arts 57-59; and martial arts teacher 59-60; motivations to 85-91; role of the teacher 95-97; social context of 94-95; well-being 69-71
Lee, Bruce 31,87

Leffers, P. 24

Levitin, D. 13

lifestyle practices: and economic resources 19-20

Lincoln,Y.S. 11

Liu, H. 23

Lodder, J. 24

Luckmann, T. 19, 97

MacPherson, H. 4

management of stress: and link to health 75-82; and link to well-being 75-82

martial arts 5; access to 43; and community $37-39$; and culture 37 ; data analysis 10-11; data collection 7-8; defined 21; and Eastern philosophy 22-26; and health 1, 21-22, 28-29, 32-34, 52-57, 53; health awareness 89-91; health benefits 88-89; identity in 97-98; impact on everyday life 35 ; improvements in well-being since beginning $\mathbf{6 5}$; interviews 9-10; Japanese 23; motivations for taking up 46-49; other benefits to practice $\mathbf{6 6}$; perceived benefits of 88 ; and physical fitness 28, 46-48; role in promoting health and well-being 5-6; and stress management 29; style in Europe 23; style in North America 23; survey 8-9; and well-being 21-22, 29-30, 34-36, 48-49, 64-82

martial arts teachers 27-44; attributes considered important in $\mathbf{4 2 , 5 9}$; on benefits of martial arts 36-40; on challenges of teaching 40-41; health and health awareness 32-34; and learning martial arts 59-60; motivations for taking up martial arts 27-32; overview 27; qualities in 60-61; on value of teaching 41-43; well-being 34-36

mastery experiences 16

Matchim,Y. 23

McLeod, S. 15

McNeill,W.H. 21, 95

mental health $3,64,66,79,82,83,91$; and martial arts 30; self-confidence and 29

Mental Health Act 3

mental ill health 3, 91, 92, 101

mental well-being 3,87-88, 94

micro-sociology 21 
MIND (British mental health charity) 64, 92

'mindfulness' 68, 72, 76

motivations to learn 85-91; general 87; health 86; health awareness 89-91; health benefits $88-89$; learning 86 ; mental well-being 87-88; perceived benefits of martial arts 88 ; physical 86

National Institute for Health 4

National Institute for Mental Health 3

Nettleton, S. 89

'No Health without Mental Health' 3

obesity: and mental health 3; and type 2 diabetes 2 ; see also diabetes

obsessive-compulsive disorder (OCD) 14 older people: and martial arts 46-47; Tai Chi 47

operant conditioning 14

Organisation for Economic Co-operation and Development (OECD) 19

perceived benefits of martial arts 88 physical confidence $66-67,78,82$

physical fitness: and martial arts 28 , 46-48; see also exercise

physical self 65-68, 86-88, 92, 103

Piano-Clark,V.L. 7

Pons van Dijk, G. 24

positive self-esteem 64

poverty: and health 13, 20; and well-being 13, 20

religion 20

research design: martial arts and health 6-7

ritualistic practices 85,94

Ross, D. 15

Ross, S.A. 15

Schwarzer, R. 18, 87

self, physical 65-68, 86-88, 92, 103

self-confidence 64,66 ; improving 87,93 ; lower levels of 78; and martial arts 29; mental well-being and 92; physical 75

self-efficacy: defined 16; and health 17-18; mastery experiences 16 ; social models 16; social persuasion 17; sources of 16-17; stress reactors 17 ; and well-being 17-18

Self-Efficacy in Changing Societies (Bandura) 17 self-esteem: low $67,78-79$; positive 64 ; subjective opinions on 92

self-mastery 85, 92-93, 103

Setton, M. 22

shared identity 94-95, 103

Skinner, B.F. 14-15, 90

social 65 ; well-being $72-75$

social cognitive theory $15-16,84$

social cohesion 18-19

social context of learning 94-95

social happiness $65,73-74$

social identity 85

social models 16

social order 19

social persuasion 17

social practices 85

social well-being 94

society: and behaviour 18-20; human beings and 85; social cohesion 18-19; see also community

Statistical Package for the Social Sciences (SPSS) software 10

stress: management of 75-82; and martial arts 29, 48; reactors 17

Stringer, L.A. 22, 23

SurveyMonkey 7

Tai Chi 24, 71; health benefits 24-25; older people 47

Taijiquan 24

Taoism 22-24, 72, 85

tastes, and economic resources 19

teacher(s): learning and 95-97; role of 95-97; see also martial arts teachers

teaching: martial arts 57-59; value of martial arts 41-43

team sports 87

Telegraph Newspaper 4

Thorogood, N. 89

type 2 diabetes 2 ; and obesity 2; see also diabetes

UK National Health Service (NHS) 1, 101; funding crisis 12; 'Public Health Responsibility Deal' 12

United Kingdom (UK): and chronic heart disease 1

United States (US): and chronic heart disease 1; medical insurance 20; and mental ill health 3

vegetarianism/veganism 83 
Wang, J. 22, 23

well-being 91-94; and alternative treatments 4 ; and culture 13 ; culture and ethics 71-72; and diabetes 2; and exercise 1 ; improvements in since beginning of martial arts $\mathbf{6 5}$; intellectual 69-72; learning 69-71; management of stress and the link to 75-82; and martial arts 21-22, 29-30, 34-36, 48-49; martial arts and well-being 64-82; mental 87-88; overview 64; and poverty 13,20 ; and self-efficacy 17-18; social 72-75; well-being and confidence 66-69
Wikipedia 71

Wong, E. 24

World Antibiotic Awareness Week 12 World Hepatitis B Day 12

World No Tobacco Day 12

Yeh, C.K. 23

Yin and Yang 24

Yu, P. 23

Yu Gongbao 96

Zen 23

Zhu Xi 25, 85, 90, 93, 98-100, 105

Zinn, H.C. 23 